A multidisciplinary approach to study virulence of the entomopathogenic fungus Beauveria bassiana towards malaria mosquitoes

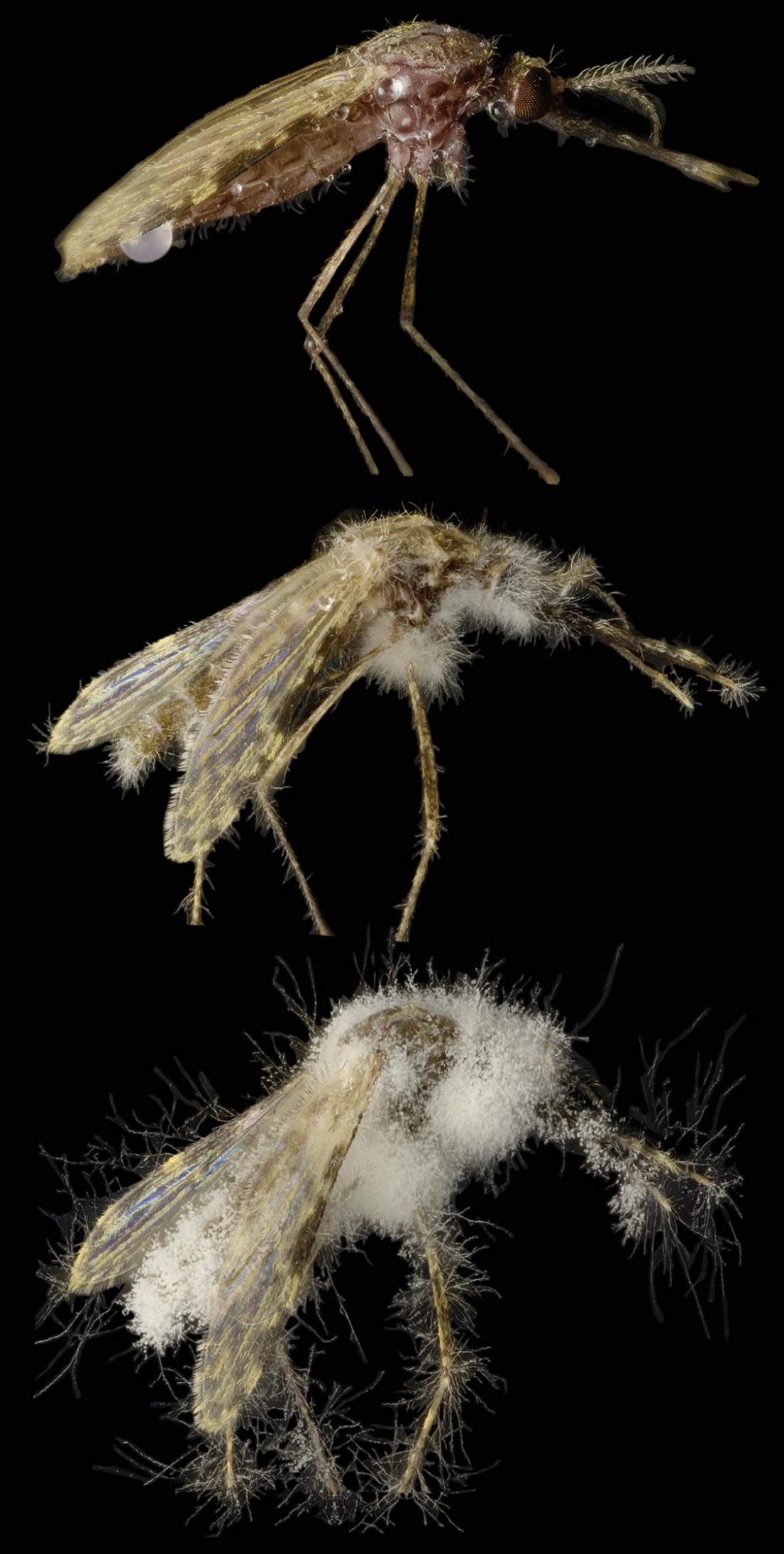

Claudio A. Valero Jiménez 


\section{A multidisciplinary approach to study virulence of the entomopathogenic fungus Beauveria bassiana towards malaria mosquitoes}

Claudio A. Valero Jiménez 


\section{Thesis committee}

\section{Promotors}

Prof. Dr B.J. Zwaan

Professor of Genetics

Wageningen University

Prof. Dr W. Takken

Personal chair at the Laboratory of Entomology

Wageningen University

\section{Co-promotors}

Dr. C.J.M. Koenraadt

Assistant professor, Laboratory of Entomology

Wageningen University

Dr. J.A.L. van Kan

Assistant professor, Laboratory of Phytopathology

Wageningen University

\section{Other members}

Prof. Dr M.E. Schranz, Wageningen University

Dr V.I.D. Ros, Wageningen University

Dr M. Rep, University of Amsterdam

Prof. Dr J. Eilenberg, University of Copenhagen, Denmark

This research was conducted under the auspices of the C.T. de Wit Graduate School for Production Ecology and Resource Conservation 


\title{
A multidisciplinary approach to study virulence of the entomopathogenic fungus Beauveria bassiana towards malaria mosquitoes
}

\author{
Claudio A. Valero Jiménez
}

\section{Thesis}

submitted in fulfilment of the requirements for the degree of doctor

at Wageningen University

by the authority of the Rector Magnificus

Prof. Dr A.P.J. Mol,

in the presence of the

Thesis Committee appointed by the Academic Board

to be defended in public

on Wednesday 21 September 2016

at 4 p.m. in the Aula. 
Claudio A. Valero Jiménez

A multidisciplinary approach to study virulence of the entomopathogenic fungus Beauveria bassiana towards malaria mosquitoes.

131 pages.

$\mathrm{PhD}$ thesis, Wageningen University, Wageningen, NL (2016)

With references, with summary in English

ISBN 978-94-6257-854-8

DOI $10.18174 / 385465$ 


\section{Table of Contents}

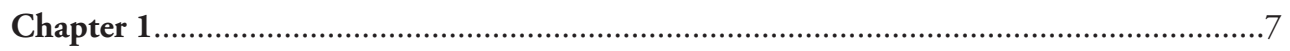

Introduction

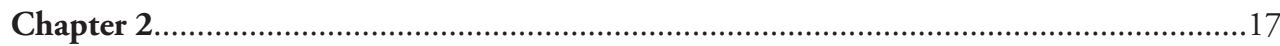

Genes involved in virulence of the entomopathogenic fungus Beauveria bassiana

Chapter 3.

Natural variation in virulence of the entomopathogenic fungus Beauveria bassiana against malaria mosquitoes

Chapter 4.

Comparative genomics of Beauveria bassiana: uncovering signatures of virulence against insects

Chapter 5 . .85

Experimental evolution to increase the efficacy of the entomopathogenic fungus Beauveria bassiana against malaria mosquitoes: effects on mycelial growth and virulence

Chapter 6.

General discussion

Summary..

Acknowledgements.

Curriculum Vitae.

Educational Statement 



\title{
Chapter 1
}

\author{
Introduction
}




\section{Chapter 1}

\section{Malaria disease}

Malaria is a vector-borne disease that causes a great threat to human health. The disease is present throughout the tropics, especially in sub-Saharan Africa. Around 214 million cases were reported in 2015, with an estimated mortality of 438 thousand people in 2014 (World Health Organization, 2015). Of these, around $70 \%$ where children under five years of age. Malaria is caused by parasites of the Plasmodium genus, most cases being caused by $P$. falciparum or $P$. vivax, and to a lesser extent by P. ovale, P. malariae, and P. knowlesi (White et al., 2014). Malaria parasites are transmitted by female mosquitoes of the genus Anopheles, but only 70 to 80 of more than 480 Anopheles species are able to do so (Manguin et al., 2008). Mosquitoes of the An. gambiae complex are considered the main vectors of human malaria in Africa and the complex is composed of eight species, namely: An. gambiae s.s., An. arabiensis, An. quadriannulatus, An. melas, An. merus, An. bwambae, An. coluzzii and An. amharicus (Coetzee, 2004; Coetzee et al., 2013).

Malaria transmission is initiated when an infected female Anopheles mosquito inoculates sporozoites into the subcutaneous tissue of a human host while blood-feeding. From there, sporozoites travel to the liver, where they undergo several transformations and eventually develop into thousands of merozoites. Once merozoites are released in the blood stream, they infect red blood cells and reproduce asexually. A small amount of asexual parasites develops into gametocytes that may be ingested by an Anopheles mosquito during a blood meal (Miller et al., 2002). Gametocytes reproduce sexually inside the mosquito, developing into a motile ookinete that migrates through the midgut wall, and becomes an oocyst on the mosquito's midgut. The oocyst grows and bursts, releasing sporozoites, which move to the salivary glands of the mosquito. When a mosquito takes a new blood meal, it will infect a new host, thereby repeating the infection cycle (White et al., 2014).

\section{Malaria control}

Presently, the main interventions to control and eliminate malaria are vector control (stop mosquitoes of infecting malaria), chemoprevention (suppression and prevention of infections) and case management (diagnosis and treatment of infections)(World Health Organization, 2015). Currently, the most effective way of eradicating and preventing malaria is achieved by vector control using insecticides (Raghavendra et al., 2011). This is accomplished through the use of Insecticide-Treated bed Nets (ITNs) and/or through Indoor Residual Spraying of insecticides (IRS). ITNs provide a physical and chemical barrier against mosquitoes and can reduce the incidence of malaria transmission, especially since most malaria mosquitoes have a nocturnal biting behaviour. ITNs are coated with pyrethroids that have a very low toxicity to 
mammals, a rapid knock-down effect, and long residual activity (Raghavendra et al., 2011). A review on interventions using ITNs has shown that they can avert the number of malaria cases by $62 \%-78 \%$ (Bhatt et al., 2015).

IRS is a method of spraying the inside of houses with residual insecticides to kill and repel malaria mosquitoes. Normally, the insecticides are sprayed on the inside walls of houses, and mosquitoes are repelled or killed when in contact with the insecticides, thus preventing the transmission of the disease (Pluess et al., 2010). IRS has also been shown to be very effective in reducing malaria transmission to similar levels as ITNs (Mabaso et al., 2004; Pluess et al., 2010).

\section{Insecticide resistance}

The progress achieved in malaria reduction using ITNs and IRS as vector control tools is challenged by the development of resistance to insecticides by malaria mosquitoes. For instance, pyrethroid resistance is of high concern since it is the only insecticide class allowed for use in ITNs and one of the four classes used in IRS (Hemingway et al., 2004; Ranson et al., 2011; Takken and Knols, 2009). Recent reports indicate that insecticide resistance is widespread across the globe with 60 countries declaring resistance to at least one class of insecticide, and 49 countries reporting resistance for two or more types of insecticide classes (Ranson and Lissenden, 2016; World Health Organization, 2015). Furthermore, long-term studies have shown that insecticides have an effect on the behaviour of mosquitoes by reducing the rate of mosquito entry into houses that result in a shift to outdoor biting and in time of biting (Mathenge et al., 2001; Mbogo et al., 1996; Reddy et al., 2011; Takken, 2002). Therefore, insecticide resistance can possibly impair the important advances accomplished through chemical malaria vector control, and consequently constrain further success.

\section{Entomopathogenic fungi: a tool for biological control of mosquitoes}

Due to the negative implications of insecticide resistance on the success of malaria control, the development of alternative tools for malaria vector control is urgently needed. There are several malaria vector control tools in development that can supplement current control methods (McGraw and O'Neill, 2013; Takken and Knols, 2009). Such tools include the modification of the environment by reducing the breeding habitats of mosquitoes or house improvement to reduce biting incidence (Imbahale et al., 2012; Kirby et al., 2008). Other vector control tools are based on genetic modification of mosquitoes, for example, the release of sterile male mosquitoes to reduce the number of offspring (Lofgren et al., 1974). Another approach is the usage of chemical compounds to attract and repel mosquitoes in a push-pull system which, when coupled with removal trapping, can reduce house entry of mosquitoes (Menger et al., 


\section{Chapter 1}

2014; Okumu et al., 2010). Finally, an additional control tool is biological control that makes use of natural enemies of insects. For instance, predatory fish as well as bacteria such as Bacillus thuringiensis var. israelensis can be used to control larval populations (Fillinger and Lindsay, 2006; Kusumawathie et al., 2008). Also entomopathogenic fungi have been used as a biological control agent of malaria mosquitoes to reduce malaria transmission (Blanford et al., 2005; Scholte et al., 2005), and this is the focus of my thesis.

Spores of entomopathogenic fungi (EPF) are able to infect mosquitoes. The time taken for mosquitoes to die after a fungal infection varies usually between three to seven days, and depends on factors such as environmental conditions, the fungal isolate used, and fitness of the host. Normally the killing time for EPF is considered to be slow compared to insecticides (1-2 days), nevertheless this is not an issue since only old mosquitoes are able to transmit malaria (Knols et al., 2010). Once a female mosquito gets infected with Plasmodium, the parasite needs around 10-14 days to mature, and only after a new blood meal they are able to infect a new human host (Killeen et al., 2000). Therefore, EPF would have sufficient time to kill older mosquitoes before they are able to transmit the parasite. Since mosquitoes will be killed at a later age, female mosquitoes can reproduce and lay eggs before dying, consequentially it will not impose a high selective pressure compared to insecticides and thus, the probability of developing resistance against fungal infections is lower (Read et al., 2009). Even if resistance could be developed, it will most likely have a fitness cost that would be only beneficial to the older cohort of the population, thus limiting the spread of resistance to the next generation (Thomas and Read, 2007).

Extensive research on EPF and malaria mosquitoes has proven that EPF are an effective tool to reduce malaria transmission under laboratory and field conditions (Blanford et al., 2005; Mnyone et al., 2012; Scholte et al., 2005). EPF are also suitable in killing insecticide-resistant mosquitoes (Farenhorst et al., 2009; Howard et al., 2011, 2010). Furthermore, EPF have an increased killing capacity towards Plasmodium infected mosquitoes, and fungal infection is able to reduce Plasmodium sporozoite levels (Blanford et al., 2005). In addition to killing abilities of EPF, infected mosquitoes have a reduced blood and sugar feeding propensity and fecundity (Ondiaka et al., 2015; Scholte et al., 2006).

\section{Infection cycle of entomopathogenic fungi}

Entomopathogenic fungi include a wide array of fungi that belong to many phylogenetic groups such as Ascomycota, Entomophthoromycota and Blastocladiomycota (Boomsma et al., 2014). Roberts and Humber (1981) listed more than 700 species across 90 genera with the capacity to infect insects. Nevertheless, throughout this thesis the term EPF is used to refer 


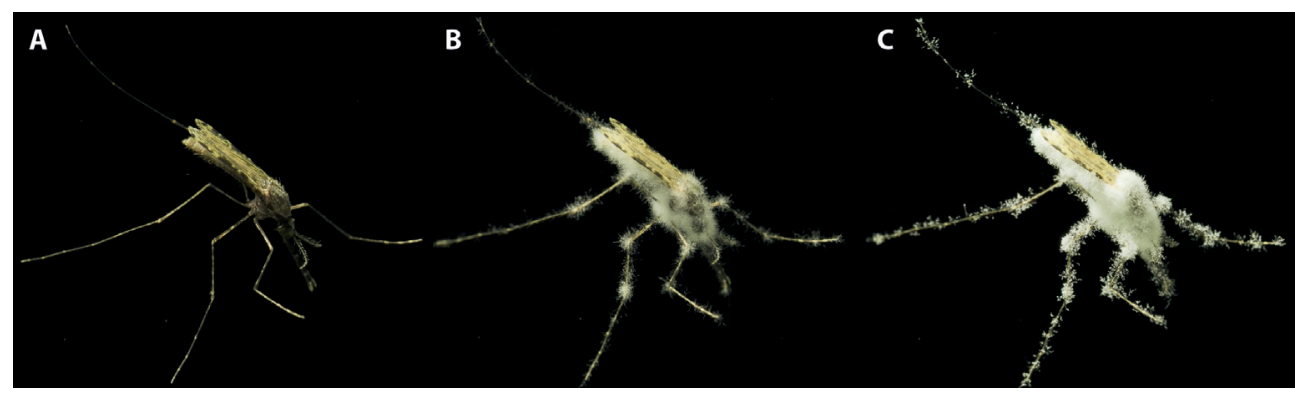

Figure 1. Fungal outgrowth of B. bassiana after the host dies. Adead mosquito after 4-6 days of being infected with $B$. bassiana spores(A).Aftertwodays, fungalmyceliagrowthoutofthebodyofmosquitoes(B) andafterfivedays, usuallymostofthebodyofthe mosquito is fully covered by mycelia and fungal spores, which areable to infect new mosquitoes (C). Source: Wimvan Egmond.

only to fungi belonging to the order Hypocreales, and more precisely to species belonging to the genera Metarhizium and Beauveria. The infection of insects by EPF starts when a conidium adheres to the host cuticle, and afterwards germinates to form a germ tube and, in some cases, an appressorium. By a combination of mechanical pressure and production of cuticle-degrading enzymes, the cuticle is penetrated and the fungus is able to reach the haemocoel (Clarkson and Charnley, 1996; Pedrini et al., 2007). Once in the haemocoel, the fungus grows as single-celled blastospores that are able to use insect nutrients and secrete toxins that eventually kill the host. For successful invasion, fungal cells need to evade and/or overcome insect immune responses during cuticle penetration and growth in the haemocoel (Barelli et al., 2015; Ortiz-Urquiza and Keyhani, 2013). When the host is killed, the fungus switches to saprophytic growth (Figure 1), and has to breach the cuticle from the inside to allow emergence from the insect body and be able to sporulate on the cadaver, thereby enabling conidia to be dispersed and start a new infection if environmental conditions are conducive (Boomsma et al., 2014).

\section{Beauveria bassiana as entomopathogenic fungus for biological control}

The EPF Beauveria bassiana was described by Agostino Bassi in the early 19th century as the causal agent of white muscardine disease of silkworms. This demonstrated for the first time that microorganisms could be the causal agents of disease. Ever since the description of this fungus, it has been used and studied for biological control of insect pest (Zimmermann, 2007). B. bassiana is a cosmopolitan ascomycete fungus that is capable of inhabiting a wide range of environments, including soil, insects, and plants. The fungus is able to live as a saprophyte in the soil, as an endophyte in plants, or as an entomopathogen affecting a broad range of arthropods (Boomsma et al., 2014). B. bassiana is haploid and normally reproduces asexually, although there are reports that it can reproduce sexually in a teleomorphic state, called Cordyceps bassiana (Sung et al., 2006). 


\section{Chapter 1}

The host range of $B$. bassiana includes more than 700 species that belong to several orders of arthropods such as Coleoptera, Diptera, Lepidoptera, and Hymenoptera (Goettel et al., 1990). Not surprisingly, there are many products on the market worldwide that include $B$. bassiana as a component of commercial mycoinsecticides (de Faria and Wraight, 2007). Moreover, $B$. bassiana is able to produce a wide array of secondary metabolites compounds implicated in insect pathogenesis and virulence that have applications in industrial, pharmaceutical, and agricultural activities. For instance, in traditional Asian medicine, dried and powdered silkworms that have died by a B. bassiana infection are used as an analgesic (Molnár et al., 2010).

\section{Virulence of entomopathogenic fungi}

Virulence and pathogenicity of agents that are able to cause disease are terms that are often confused and/or even misused in the scientific literature (Bos and Parlevliet, 1995; Thomas and Elkinton, 2004). Throughout this thesis, I will adhere to the definitions proposed by ShapiroIlan et al. (2005) that are based on widely used insect pathology literature. Pathogenicity is the quality or state of being pathogenic, and virulence is the disease producing power of an organism. In other words, pathogenicity is a binary variable (yes/no), for instance bacterium $\mathrm{X}$ is pathogenic to host $\mathrm{Y}$, but is not pathogenic (is not able to cause disease) in host Z. Virulence is a quantitative measure of the ability of a specific amount of an agent to cause disease (Shapiro-Ilan et al., 2012). So, if virus $\mathrm{C}$ is able to kill quicker than virus $\mathrm{A}$ at the same concentration, virus $\mathrm{C}$ is defined as being more virulent. In general, a more virulent pathogen requires fewer infective units (e.g. spores, cells, toxins), to cause disease relative to a pathogen that is less virulent.

\section{A better understanding of virulence is urgently needed}

To assess whether a biological control approach that makes use of EPF, is a robust and sustainable solution to kill malaria mosquitoes, we need a better understanding of the life history of the biological control agent as well as the mechanisms and modes of action in killing the host. However, fundamental knowledge on the mechanisms and regulation of the infection process in the fungus, as well as insights into the defensive responses of the host insect to EPF, is limited. A better understanding of these aspects will infer whether development of resistance in EPF is likely to occur. Only then can mass application of fungi be considered as an effective tool for the control of mosquito-borne diseases. 


\section{Introduction}

\section{Outline of this thesis}

The main goal of this thesis was to study virulence of the entomopathogenic fungus $B$. bassiana towards malaria mosquitoes using a multidisciplinary approach. Chapter 2 provides an overview of existing knowledge of genes influencing virulence in EPF, with a special focus on B. bassiana. The infection cycle and virulence mechanisms are discussed, and put in a framework of novel strategies and experimental methods that are needed to better understand virulence and improve the usage of EPF as a biocontrol agent.

The study of natural variation in fungal virulence is a first step towards understanding the genetic mechanisms involved, because it reveals the extent of variation in the different components of virulence and their overall role. Chapter 3 describes the natural variation in virulence for 29 B. bassiana isolates that were tested on malaria mosquitoes. Furthermore, the phenotypic characteristics of the fungal isolates such as sporulation, spore size and growth were evaluated and their relationship with virulence analysed.

Based on the ample natural variation observed in fungal virulence, in Chapter $\mathbf{4}$, a comparative genomics analysis was performed on five selected isolates of contrasting virulence. In order to understand mechanisms underlying contrasting virulence, a comparison on gene gain/loss, single nucleotide polymorphisms (SNPs), secreted proteins, and secondary metabolites was performed. Insight is provided to the magnitude of the complexity of a trait such as virulence and suggests candidate genes that can be further studied using a functional analysis approach.

Chapter 5 focuses on an experimental evolution approach in which B. bassiana was solely using insects as a nutritional source for ten consecutive passages through malaria mosquitoes. Two isolates of $B$. bassiana that differed in virulence were compared to their respective ancestors, and they were assayed in virulence, fungal outgrowth, mycelial growth rate (MGR), and sporulation. Passage of the entomopathogenic fungi $B$. bassiana through the insect host resulted in an altered capacity to grow on different substrates while maintaining the ability to kill insects.

Chapter 6 presents a discussion on the main findings of this thesis and describes future perspectives to study virulence of the entomopathogenic fungi Beauveria bassiana in the context of biological control of malaria mosquitoes. 


\section{Chapter 1}

\section{References}

Barelli, L., Moonjely, S., Behie, S.W., Bidochka, M.J., 2015. Fungi with multifunctional lifestyles: endophytic insect pathogenic fungi. Plant Mol Biol. doi:10.1007/ s11103-015-0413-z

Bhatt, S., Weiss, D.J., Cameron, E., Bisanzio, D., Mappin, B., Dalrymple, U., Battle, K.E., Moyes, C.L., Henry, A., Eckhoff, P.A., Wenger, E.A., Briet, O., Penny, M.A., Smith, T.A., Bennett, A., Yukich, J., Eisele, T.P., Griffin, J.T., Fergus, C.A., Lynch, M., Lindgren, F., Cohen, J.M., Murray, C.L.J., Smith, D.L., Hay, S.I., Cibulskis, R.E., Gething, P.W., 2015. The effect of malaria control on Plasmodium falciparum in Africa between 2000 and 2015. Nature 526, 207-211. doi:10.1038/nature15535

Blanford, S., Chan, B.H.K., Jenkins, N., Sim, D., Turner, R.J., Read, A.F., Thomas, M.B., 2005. Fungal pathogen reduces potential for malaria transmission. Science 308, 1638-41. doi:10.1126/science. 1108423

Boomsma, J.J., Jensen, A.B., Meyling, N.V., Eilenberg, J., 2014. Evolutionary interaction networks of insect pathogenic fungi. Annu Rev Entomol 59, 467-485. doi:10.1146/annurev-ento-011613-162054

Bos, L., Parlevliet, J.E., 1995. Concepts and Terminology on Plant Pest Relationships: Toward Consensus in Plant Pathology and Crop Protection. Annu Rev Phytopathol 33, 69-102.

Clarkson, J.M., Charnley, A.K., 1996. New insights into the mechanisms of fungal pathogenesis in insects. Trends Microbiol 4, 197-203.

Coetzee, M., 2004. Distribution of the African malaria vectors of the Anopheles gambiae complex. Am J Trop Med Hyg 70, 103-4. doi:70/2/103 [pii]

Coetzee, M., Hunt, R.H., Wilkerson, R., Torre, A. Della, Coulibaly, M.B., Besansky, N.J., 2013. Anopheles coluzzii and Anopheles amharicus, new members of the Anopheles gambiae complex. Zootaxa 3619, 246-274. doi:10.11646/zootaxa.3619.3.2

de Faria, M.R., Wraight, S.P., 2007. Mycoinsecticides and Mycoacaricides: A comprehensive list with worldwide coverage and international classification of formulation types. Biol Control 43, 237-256. doi:10.1016/j. biocontrol.2007.08.001

Farenhorst, M., Mouatcho, J.C., Kikankie, C.K., Brooke, B.D., Hunt, R.H., Thomas, M.B., Koekemoer, L.L., Knols, B.G.J., Coetzee, M., 2009. Fungal infection counters insecticide resistance in African malaria mosquitoes. Proc Natl Acad Sci U S A 106, 1744317447. doi:10.1073/pnas.0908530106

Fillinger, U., Lindsay, S.W., 2006. Suppression of exposure to malaria vectors by an order of magnitude using microbial larvicides in rural Kenya. Trop Med Int Heal 11,
1629-1642. doi:10.1111/j.1365-3156.2006.01733.x

Goettel, M.S., Poprawski, T.J., Vandenberg, J.. D., Li, Z., Roberts, D.W., 1990. Safety to nontarget invertebrates of fungal biocontrol agents. Saf Microb Insectic 209-231.

Hemingway, J., Hawkes, N.J., McCarroll, L., Ranson, H., 2004. The molecular basis of insecticide resistance in mosquitoes. Insect Biochem Mol Biol 34, 653-665. doi:10.1016/j.ibmb.2004.03.018

Howard, A.F. V, Koenraadt, C.J.M., Farenhorst, M., Knols, B.G.J., Takken, W., 2010. Pyrethroid resistance in Anopheles gambiae leads to increased susceptibility to the entomopathogenic fungi Metarhizium anisopliae and Beauveria bassiana. Malar J 9, 168. doi:10.1186/14752875-9-168

Howard, A.F. V, N'Guessan, R., Koenraadt, C.J.M., Asidi, A., Farenhorst, M., Akogbéto, M., Knols, B.G.J., Takken, W., 2011. First report of the infection of insecticide-resistant malaria vector mosquitoes with an entomopathogenic fungus under field conditions. Malar J 10, 24. doi:10.1186/1475-2875-10-24

Imbahale, S.S., Githeko, A., Mukabana, W.R., Takken, W., 2012. Integrated mosquito larval source management reduces larval numbers in two highland villages in western Kenya. BMC Public Health 12, 362. doi:10.1186/14712458-12-362

Killeen, G.F., McKenzie, F.E., Foy, B.D., Schieffelin, C., Billingsley, P.F., Beier, J.C., 2000. A simplified model for predicting malaria entomologic inoculation rates based on entomologic and parasitologic parameters relevant to control. Am J Trop Med Hyg 62, 535-44.

Kirby, M.J., West, P.A., Green, C., Jasseh, M., Lindsay, S.W., 2008. Risk factors for house-entry by culicine mosquitoes in a rural town and satellite villages in The Gambia. Parasites and Vectors 1, 41. doi:10.1186/17563305-1-41

Knols, B.G.J., Bukhari, T., Farenhorst, M., 2010. Entomopathogenic fungi as the next-generation control agents against malaria mosquitoes. Future Microbiol 5, 339-341. doi: $10.2217 / \mathrm{fmb} .10 .11$

Kusumawathie, P.H., Wickremasinghe, A.R., Karunaweera, N.D., Wijeyaratne, M.J., 2008. Larvivorous potential of the guppy, Poecilia reticulata, in anopheline mosquito control in riverbed pools below the Kotmale dam, Sri Lanka. Asia Pac J Public Heal 20, 56-63. doi:10.1177/1010539507308507

Lofgren, C.S., Dame, D.A., Breeland, S.G., Weidhaas, D.E., Jeffery, G., Kaiser, R., Ford, H.R., Boston, M.D., Baldwin, K.F., 1974. Release of chemosterilized males for the control of Anopheles albimanus in El Salvador. III. Field methods and population control. Am J Trop Med Hyg 23, 288-297.

Mabaso, M.L.H., Sharp, B., Lengeler, C., 2004. Historical 
review of malarial control in southern African with emphasis on the use of indoor residual house-spraying. Trop Med Int Heal 9, 846-856. doi:10.1111/j.13653156.2004.01263.x

Manguin, S., Carnevale, P., Mouchet, J., Coosemans, M., Julvez, J., Richard-Lenoble, D., Sircoulon, J., 2008. Biodiversity of malaria in the world. John Libbey Eurotext.

Mathenge, E.M., Gimnig, J.E., Kolczak, M., Ombok, M., Irungu, L.W., Hawley, W. a, 2001. Effect of permethrinimpregnated nets on exiting behavior, blood feeding success, and time of feeding of malaria mosquitoes (Diptera: Culicidae) in western Kenya. J Med Entomol 38, 531-536. doi:10.1603/0022-2585-38.4.531

Mbogo, C.N., Baya, N.M., Ofulla, A. V, Githure, J.I., Snow, R.W., 1996. The impact of permethrin-impregnated bednets on malaria vectors of the Kenyan coast. Med Vet Entomol 10, 251-9. doi:10.1111/j.1365-2915.1996. tb00739.x

McGraw, E. A., O’Neill, S.L., 2013. Beyond insecticides: new thinking on an ancient problem. Nat Rev Microbiol 11, 181-93. doi:10.1038/nrmicro2968

Menger, D.J., Otieno, B., de Rijk, M., Mukabana, W.R., van Loon, J.J., Takken, W., 2014. A push-pull system to reduce house entry of malaria mosquitoes. Malar J 13, 119. doi:10.1186/1475-2875-13-119

Miller, L.H., Baruch, D.I., Marsh, K., Doumbo, O.K., 2002. The pathogenic basis of malaria. Nature 415, 673679. doi:10.1038/415673a

Mnyone, L.L., Lyimo, I.N., Lwetoijera, D.W., Mpingwa, M.W., Nchimbi, N., Hancock, P.A., Russell, T.L., Kirby, M.J., Takken, W., Koenraadt, C.J.M., 2012. Exploiting the behaviour of wild malaria vectors to achieve high infection with fungal biocontrol agents. Malar J 11, 87. doi:10.1186/1475-2875-11-87

Molnár, I., Gibson, D.M., Krasnoff, S.B., 2010. Secondary metabolites from entomopathogenic Hypocrealean fungi. Nat Prod Rep 27, 1241-1275. doi:10.1039/ c001459c

Okumu, F.O., Killeen, G.F., Ogoma, S., Biswaro, L., Smallegange, R.C., Mbeyela, E., Titus, E., Munk, C., Ngonyani, H., Takken, W., Mshinda, H., Mukabana, W.R., Moore, S.J., 2010. Development and field evaluation of a synthetic mosquito lure that is more attractive than humans. PLoS One 5. doi:10.1371/ journal.pone.0008951

Ondiaka, S.N., Masinde, E.W., Koenraadt, C.J., Takken, W., Mukabana, W.R., 2015. Effects of fungal infection on feeding and survival of Anopheles gambiae (Diptera: Culicidae) on plant sugars. Parasit Vectors 8, 1-11. doi:10.1186/s13071-015-0654-3

Ortiz-Urquiza, A., Keyhani, N.O., 2013. Action on the surface: Entomopathogenic fungi versus the insect cuticle. Insects 4, 357-374. doi:10.3390/insects4030357

Pedrini, N., Crespo, R., Juárez, M.P., 2007. Biochemistry of insect epicuticle degradation by entomopathogenic fungi. Comp Biochem Physiol C Toxicol Pharmacol 146, 124-137. doi:10.1016/j.cbpc.2006.08.003

Pluess, B., Tanser, F.C., Lengeler, C., Sharp, B.L., 2010. Indoor residual spraying for preventing malaria, in: Lengeler, C. (Ed.), Cochrane Database of Systematic Reviews. John Wiley \& Sons, Ltd, Chichester, UK. doi:10.1002/14651858.CD006657.pub2

Raghavendra, K., Barik, T.K., Reddy, B.P.N., Sharma, P., Dash, A.P., 2011. Malaria vector control: from past to future. Parasitol Res 108, 757-779. doi:10.1007/ s00436-010-2232-0

Ranson, H., Lissenden, N., 2016. Insecticide Resistance in African Anopheles Mosquitoes: A Worsening Situation that Needs Urgent Action to Maintain Malaria Control. Trends Parasitol 32, 187-196. doi:10.1016/j. pt.2015.11.010

Ranson, H., N'Guessan, R., Lines, J., Moiroux, N., Nkuni, Z., Corbel, V., 2011. Pyrethroid resistance in African anopheline mosquitoes: What are the implications for malaria control? Trends Parasitol 27, 91-98. doi:10.1016/j.pt.2010.08.004

Read, A.F., Lynch, P. a, Thomas, M.B., 2009. How to make evolution-proof insecticides for malaria control. PLoS Biol 7, e1000058. doi:10.1371/journal.pbio. 1000058

Reddy, M.R., Overgaard, H.J., Abaga, S., Reddy, V.P., Caccone, A., Kiszewski, A.E., Slotman, M. a, 2011. Outdoor host seeking behaviour of Anopheles gambiae mosquitoes following initiation of malaria vector control on Bioko Island, Equatorial Guinea. Malar J 10, 184. doi:10.1186/1475-2875-10-184

Roberts, D.W., Humber, R.A., 1981. Biology of Conidial Fungi, Biology of Conidial Fungi. Elsevier. doi:10.1016/ B978-0-12-179502-3.50014-5

Scholte, E.-J., Knols, B.G.J., Takken, W., 2006. Infection of the malaria mosquito Anopheles gambiae with the entomopathogenic fungus Metarhizium anisopliae reduces blood feeding and fecundity. J Invertebr Pathol 91, 43-9. doi:10.1016/j.jip.2005.10.006

Scholte, E.-J., Ng'habi, K., Kihonda, J., Takken, W., Paaijmans, K., Abdulla, S., Killeen, G.F., Knols, B.G.J., 2005. An entomopathogenic fungus for control of adult African malaria mosquitoes. Science (80- ) 308, 1641-2. doi:10.1126/science.1108639

Shapiro-Ilan, D.I., Bruck, D.J., Lacey, L.A., 2012. Insect Pathology. Elsevier. doi:10.1016/B978-0-12-3849847.00003-8

Shapiro-Ilan, D.I., Fuxa, J.R., Lacey, L.A., Onstad, D.W., 


\section{Chapter 1}

Kaya, H.K., 2005. Definitions of pathogenicity and virulence in invertebrate pathology. J Invertebr Pathol 88, 1-7. doi:10.1016/j.jip.2004.10.003

Sung, J.-M., Lee, J.-O., Humber, R.A., Sung, G.-H., Shrestha, B., 2006. Cordyceps bassiana and Production of Stromata in vitro Showing Beauveria Anamorph in Korea. Mycobiology 34, 1. doi:10.4489/MYCO.2006.34.1.001

Takken, W., 2002. Do insecticide treated bednets have an effect on malaria vectors? Trop Med Int Heal 7, 10221030.

Takken, W., Knols, B.G.J., 2009. Malaria vector control: current and future strategies. Trends Parasitol 25, 101-4. doi:10.1016/j.pt.2008.12.002

Thomas, M.B., Read, A.F., 2007. Can fungal biopesticides control malaria? Nat Rev Microbiol 5, 377-383. doi: $10.1038 /$ nrmicro 1638

Thomas, S.R., Elkinton, J.S., 2004. Pathogenicity and virulence. J Invertebr Pathol. doi:10.1016/j. jip.2004.01.006

White, N.J., Pukrittayakamee, S., Hien, T.T., Faiz, M.A., Mokuolu, O.A., Dondorp, A.M., 2014. Malaria. Lancet 383, 723-735. doi:10.1016/S0140-6736(13)60024-0

World Health Organization, 2015. World Malaria Report 2014 Summary.

Zimmermann, G., 2007. Review on safety of the entomopathogenic fungi Beauveria bassiana and Beauveria brongniartii. Biocontrol Sci Technol 17, 553596. doi:10.1080/09583150701309006 


\section{Chapter 2}

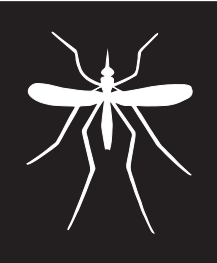

\section{Genes involved in virulence of the entomopathogenic fungus Beauveria bassiana}

Claudio A. Valero-Jiménez ${ }^{1,2}$, Harm Wiegers ${ }^{1}$, Bas J. Zwaan ${ }^{1}$, Constantianus J.M. Koenraadt ${ }^{2}$ and Jan A.L. van Kan ${ }^{3}$

${ }^{1}$ Laboratory of Genetics, Wageningen University, P.O. Box 16, 6700 AA Wageningen, The Netherlands ${ }^{2}$ Laboratory of Entomology, Wageningen University, P.O. Box 16, 6700 AA Wageningen, The Netherlands

${ }^{3}$ Laboratory of Phytopathology, Wageningen University, P.O. Box 16, 6700 AA Wageningen, The Netherlands 


\title{
Chapter 2
}

\begin{abstract}
Pest insects cause severe damage to global crop production and pose a threat to human health by transmitting diseases. Traditionally, chemical pesticides (insecticides) have been used to control such pests and have proven to be effective only for a limited amount of time because of the rapid spread of genetic insecticide resistance. The basis of this resistance is mostly caused by (co) dominant mutations in single genes, which explains why insecticide use alone is an unsustainable solution. Therefore, robust solutions for insect pest control need to be sought in alternative methods such as biological control agents for which single-gene resistance is less likely to evolve. The entomopathogenic fungus Beauveria bassiana has shown potential as a biological control agent of insects, and insight into the mechanisms of virulence is essential to show the robustness of its use. With the recent availability of the whole genome sequence of $B$. bassiana, progress in understanding the genetics that constitute virulence toward insects can be made more quickly. In this review we divide the infection process into distinct steps and provide an overview of what is currently known about genes and mechanisms influencing virulence in $B$. bassiana. We also discuss the need for novel strategies and experimental methods to better understand the infection mechanisms deployed by entomopathogenic fungi. Such knowledge can help improve biocontrol agents, not only by selecting the most virulent genotypes, but also by selecting the genotypes that use combinations of virulence mechanisms for which resistance in the insect host is least likely to develop.
\end{abstract}

\section{Keywords}

Entomopathogenic fungi; Beauveria bassiana; Virulence genes; Virulence factors 


\section{Introduction}

Arthropod pests are a nuisance for human health and global production of crops used for food, biofuel, and fibers. Different insect species act as vectors that transmit lethal diseases, including malaria and dengue. Malaria has the highest death rate of all vector-borne diseases, with 584,000 estimated mortalities in 2013 (WHO, 2014). On the other hand, dengue is the fastest increasing vector-borne disease, with a global estimate of 390 million new infections annually (Bhatt et al., 2013). Control of disease vectors is an important means to prevent infection and spreading of diseases because these vectors can be the most vulnerable or preventable stage of human disease development (Zaim and Guillet, 2002). Other insect pests cause serious damage to crops grown throughout the world. Reduction of the annual global crop yield by insect pests was estimated to amount to $18 \%$ a decade ago, apart from loss of stored grains (Bergvinson and García-Lara, 2004 and Oerke and Dehne, 2004). A more recent study estimated a loss of about $8 \%$ of the major crops in Brazil due to pests, in spite of control measures (Oliveira et al., 2014).

Though chemical pesticides are widely applied in agriculture since many decades, several complications undermine their applicability, such as acquired insecticide resistance, serious cases of pesticide poisoning, and environmental pollution (Oliveira et al., 2014 and Zaim and Guillet, 2002). There is a growing urgency to develop alternative pesticides, and biopesticides are of promising potential. Various entomopathogenic fungi, such as Beauveria bassiana and Metarhizium spp., are currently used as biological control agents for different arthropods, both to fight the dispersal of diseases and to reduce the loss of crops or harvested produce (de Faria and Wraight, 2007).

The cosmopolitan ascomycete fungus B. bassiana is capable of exploiting a range of environments, including soil, plants and insects; the fungus is able to live as a saprophyte in the soil, as an endophyte in plants, or as an entomopathogen affecting a broad range of arthropods (Boomsma et al., 2014). It can reproduce sexually in a teleomorphic state called Cordyceps bassiana (Sung et al., 2006). Conidia can start an infection upon contact with a compatible insect host, then colonize the host and ultimately kill it. B. bassiana insect hosts include crop pests (e.g. whiteflies and borers, (Brownbridge et al., 2001 and Cruz et al., 2006)), ecologically hazardous pests (e.g. termites, (Jones et al., 1996)) and insect disease vectors, including mosquitoes and ticks (Blanford et al., 2005 and Kirkland et al., 2004). Its wide host range makes B. bassiana a suitable organism to control a variety of pests (Zhang et al., 2011b), as long as specific delivery tools are used to target only the pest species.

To further assess whether a biocontrol approach that makes use of entomopathogenic fungi 


\section{Chapter 2}

is a robust and sustainable solution to arthropod pests, we need a better understanding of the life history of the biocontrol agent as well as the mechanisms and modes of action in killing the pest. With the recent availability of the whole genome sequence of $B$. bassiana (Xiao et al., 2012), rapid progress can be made in understanding the genetics that constitute virulence toward insects. This review provides an overview of our current knowledge of the genes contributing to virulence in entomopathogenic fungi, with an emphasis on $B$. bassiana. Current insights in virulence mechanisms are discussed, implicating possibilities to increase the efficiency and killing speed of $B$. bassiana strains in order to improve their potential as a biopesticide in the fight against crop loss and disease dispersal by insects.

\section{Insect infection cycle by $B$. bassiana}

This review focuses on the infection cycle of $B$. bassiana in insects, which can be divided into seven steps (Fig. 1). To discuss the life cycle of B. bassiana is beyond the scope of this article, but see Boomsma et al. (2014) for a complete overview. To enable infection, conidia must adhere to the host cuticle (step 1) and subsequently germinate to form a germ tube and in particular cases an appressorium (2). The cuticle can then be breached using a combination of mechanical pressure and enzymatic degradation (3), enabling hyphae to grow through the cuticle and invade the insect haemocoel, where they switch to growth as single-celled blastospore (4). These cells circulate freely through the haemocoel, where they exploit the haemolymph for nutrition and secrete toxins that eventually kill the host (5). Successful invasion requires that the fungal cells evade or overcome immune responses (6), both during growth through the cuticle and especially in the haemocoel. When the host is killed, the cuticle must be breached again from the inside to allow escape from the insect body and sporulate on the cadaver (7), enabling conidia to be dispersed and start a new infection. We will discuss each of the steps in more detail and describe what is currently known about genes that play a role in each of the steps of the B. bassiana infection cycle (summarized in Table 1). Most of this information results from the phenotypic characterization of targeted knock-out mutants. Due to the complex life history of B. bassiana, many of the virulence genes studied are involved in more than one step of the infection cycle, or they are relevant for fundamental processes such as conidiation and cell wall formation. In cases where the deletion of a candidate virulence gene visibly affects the growth rate and cellular development of the fungus in vitro, it is difficult to draw conclusions about the specific function of a gene in the infection process, since developmental defects may impinge on the virulence in an indirect manner. For completeness, we have nevertheless included information on pleiotropic developmental genes in our list of "virulence genes". 


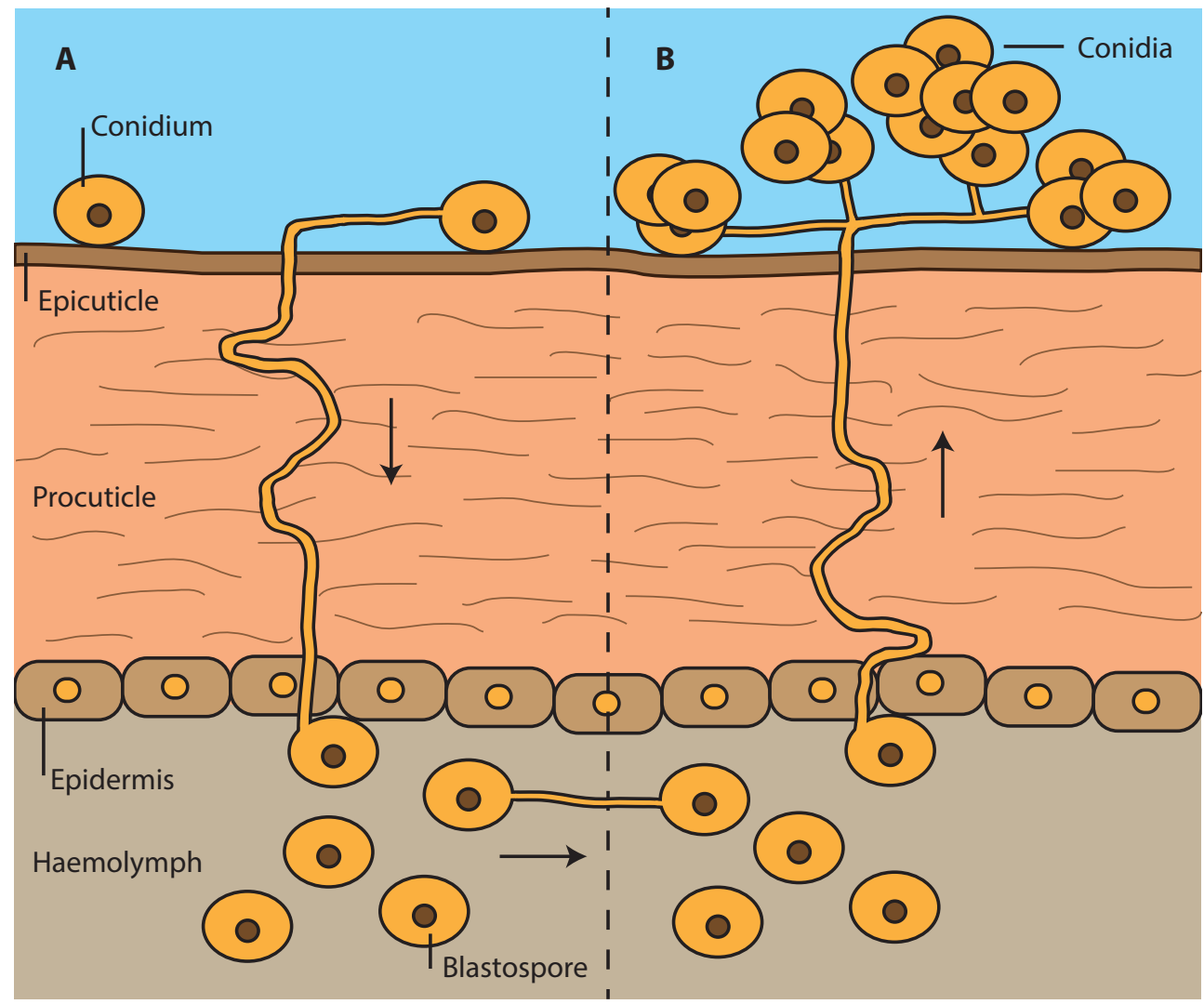

Figure 1. Infection cycle of Beauveria bassiana in insects (adapted from Clarkson and Charnley, 1996). (A) A conidium must adhere to the insect host to induce germination (step 1). This is followed by germination and production of a germ tube (step 2). Mechanical pressure and secretion of enzymes are employed to breach the cuticle (step 3). The fungus switches to blastospore growth to colonize the host haemocoel (step 4), where it secretes toxins and feeds on sugars in the haemolymph (step 5). The blastospores evade or overcome host immune responses and secrete toxins that facilitate killing of the host (step 6). (B) After the host has died, the fungus breaches the cuticle again from the inside and sporulates on the cadaver (step 7). The arrows indicate the direction of fungal growth.

\subsection{Host adherence}

The primary route for successful infection is adherence of a conidium to the host. B. bassiana aerial conidia have a hydrophobic coating and adhere to an insect cuticle through hydrophobic surface interactions. Passive adhesion can be mediated by hydrophobins (Holder and Keyhani, 2005). B. bassiana expresses two hydrophobin genes, hyd 1 and $h y d 2$, encoding proteins that accumulate and covalently aggregate on the surface of conidia. The $\Delta$ hyd1 1 hyd 2 double knockout mutants showed reduced conidial adhesion and decreased virulence, though both genes have different functions. Whereas the $\Delta h y d 2$ knock-out mutant conidia showed reduced surface adhesion but unchanged virulence, the $\Delta h y d 1$ knock-out mutant displayed decreased virulence 


\section{Chapter 2}

but unchanged surface adhesion (Zhang et al., 2011b).

Apart from hydrophobicity, electrical charge of the conidia is thought to affect adhesion. Conidial surfaces have a net negative charge that attracts them toward positively charged surfaces. However, neutralization of the conidial surface charge did not inhibit binding of conidia to the insect cuticle (Boucias et al., 1988).

The compositions of cuticle and conidial surfaces influence adhesion potential. For example, fatty amides in the cuticle of the insect host Liposcelis bostrychophila reduce conidial adhesion, by decreasing electrical charge and hydrophobicity (Lord and Howard, 2004). In a similar way, the composition of proteins and sugars on the conidial surface can affect their adhesion through charge and hydrophobicity. MAD1 and MAD2 are two adhesin-like proteins identified in Metarhizium anisopliae. These proteins, present on the conidial surface, were found to be required for adhesion to insect cuticle and plant epidermis, respectively. In addition, MAD1 influences germination (step 2), blastospore formation (step 5) and, ultimately, virulence (Wang and St. Leger, 2007a). Homologs have been found in B. bassiana, though their functions remain to be elucidated (Xiao et al., 2012).

\subsection{Germination and penetration peg development}

Once aerial conidia have adhered to the host cuticle, they can germinate when the right conditions are met. These conditions include favourable temperature and humidity, and the presence of an exogenous carbon source (Bidochka et al., 2000). Germinated conidia grow germ tubes, that develop into a penetration peg that perforates the cuticle to invade the host. Little is known about genes that are involved in host sensing and regulating the differentiation of penetration pegs. In some strains, it was reported that germlings differentiate into appressoria, although the formation of an appressorium structure is not required for a successful infection (Wagner and Lewis, 2000). In B. bassiana strain Bb0062, the MAP kinase gene Bbmpk1 was shown to be required for appressorium formation and penetration on cicada wings ( $Z$ hang et al., 2010). Also, the disruption of the MAP kinase gene Bbhog1 resulted in reduced frequency of appressorium formation and reduced virulence (Zhang et al., 2009). MAP kinases affect a number of processes in entomopathogenic fungi, including conidial adhesion (step 1), appressorium formation and virulence. Regulators, such as MAP kinases, affect the expression of several downstream genes, and their disruption has pleiotropic effects. Such studies therefore do not elucidate which target genes are specifically involved in appressorium formation. More targeted studies are required to unravel how appressoria (if they are formed) contribute to virulence. 
Although appressorium formation in B. bassiana is not prevalent and their role in virulence is not clear, the formation of an appressorium in Metarhizium species is clearly established and several studies have been performed on genes involved in their formation. The gene mpll (Metarhizium perilipin-like protein) was shown to function in the storage of lipids in droplets, especially in appressoria. Delayed appressorium formation and reduced turgor pressure were observed in knock-out mutants in Mapls1, a gene highly expressed in appressoria (Luo et al., 2013). Appressoria of the $\Delta m p l l$ knock-out mutant showed strongly reduced turgor pressure and impaired ability to breach the cuticle (Wang and St. Leger, 2007b). Knock-out mutants in the response regulator $M r s k n 7$ also were impaired in virulence, resulting from inability to form appressoria (Shang et al., 2015). Deletion of the gene Magas1 resulted in reduced cuticle penetration, though appressorium formation was unaffected (Cao et al., 2012). A recurring finding in these studies was that the reduction of virulence was only observed upon topical application, but not upon intrahaemocoel application of conidia, indicating fundamental roles of these genes in cuticle penetration.

\subsection{Enzymatic cuticle degradation}

Germ tubes on the cuticle are subject to conditions of environmental stress, such as high temperature and UV exposure, and to insect defence responses in the cuticle. Accelerated breaching of the cuticle may reduce the time of exposure to these conditions and thereby increase the chance of successful infection, thus positively contributing to the virulence of the fungus (Zhang et al., 2010). Penetration of the cuticle is not enabled solely by mechanical pressure, but is also facilitated by simultaneous enzymatic degradation of the cuticle.

The cuticle consists mainly of a framework of chitin, supporting proteins and lipids. The proportions of these three components in the migratory grasshopper Melanoplus sanguinipes were circa 30\%, $61 \%$ and $7.4 \%$, respectively (Bidochka and Khachatourians, 1992). Entomopathogenic fungi secrete various enzymes to degrade these cuticle components, mainly proteases and chitinases (Charnley and St. Leger, 1991). The cuticle is degraded both to obtain nutrients and to weaken the structural barrier, enabling host invasion.

The outermost, waxy layer of the epicuticle is rich in lipids, including fatty acids and hydrocarbons. The hydrocarbons present in the epicuticle mainly include alkenes, methyl-branched chains and $\mathrm{n}$-alkanes, the latter of which are sufficient to serve as a carbon source for the fungus. Insects can change the hydrocarbon content of the cuticle in response to fungal infection, which may influence the success of infection, as different hydrocarbons can either inhibit or stimulate conidial adhesion, germination and growth (Pedrini et al., 2013). 


\section{Chapter 2}

Table 1. Overview of discussed B. bassiana genes involved in virulence, in order of the main affected infection step $(1=$ host adhesion, $2=$ germination, $3=$ cuticle degradation, $4=$ growth as blastospores, 5 = host colonization and killing, $6=$ immune response interactions, $7=$ hyphal extrusion and conidiation).

\begin{tabular}{|c|c|c|c|c|}
\hline Gene name & Step & Characteristics & Knock-out mutant phenotype & References \\
\hline hyd1 & 1 & Hydrophobin & Reduced virulence & Zhang et al. (2011b) \\
\hline hyd2 & 1 & Hydrophobin & Reduced host adherence & Zhang et al. (2011b) \\
\hline Bbhog1 & $1,2,6,7$ & MAP kinase & $\begin{array}{l}\text { Reduced virulence, conidial yield, host adherence, } \\
\text { appressoria and stress-tolerance }\end{array}$ & Zhang et al. (2009) \\
\hline Bbmpk1 & $1,2,7$ & MAP kinase & $\begin{array}{c}\text { No appressoria, reduced cuticle breaching, host } \\
\text { adherence and conidiation }\end{array}$ & Zhang et al. (2010) \\
\hline Bbsnf1 & $2,4,7$ & Protein kinase & $\begin{array}{l}\text { Reduced virulence, acidification, conidiation and } \\
\text { blastospore yield }\end{array}$ & Wang et al. (2014c) \\
\hline Bbcyp $52 \times 1$ & $2,3,7$ & Cytochrome P450 & $\begin{array}{c}\text { Reduced virulence, germination and cuticle } \\
\text { breaching }\end{array}$ & Zhang et al. (2012) \\
\hline cdep 1 & 3,7 & $\begin{array}{l}\text { Subtilisin-like } \\
\text { protease }\end{array}$ & - & Fang et al. (2008) \\
\hline $\operatorname{Pr} 1, \operatorname{Pr} 2$ & 3,7 & $\begin{array}{l}\text { Subtilisin-like } \\
\text { protease }\end{array}$ & - & $\begin{array}{l}\text { Joshi et al., } 1995 \text { and } \\
\text { Dias et al., } 2008\end{array}$ \\
\hline Bbchit1 & 3,7 & Chitinase & Overexpression increased infection efficiency & Fang et al. (2005) \\
\hline $\begin{array}{l}\text { Chi1, Chi2, } \\
\text { ChsA2 }\end{array}$ & 3,7 & Chitinase & - & Liu et al. (2013) \\
\hline $\operatorname{cdc} 25$ & $4,6,7$ & $\begin{array}{l}\text { Regulates Cdk1 } \\
\text { activity }\end{array}$ & $\begin{array}{l}\text { Altered cell morphology, reduced virulence, } \\
\text { conidiation and stress-tolerance }\end{array}$ & Qiu et al. (2015) \\
\hline wee1 & $4,6,7$ & $\begin{array}{l}\text { Regulates Cdk1 } \\
\text { activity }\end{array}$ & $\begin{array}{l}\text { Altered cell morphology, reduced virulence, } \\
\text { conidiation and stress-tolerance }\end{array}$ & Qiu et al. (2015) \\
\hline Fkh2 & $4,6,7$ & Transcription factor & $\begin{array}{l}\text { Altered cell morphology, reduced virulence, } \\
\text { conidiation and stress-tolerance }\end{array}$ & Wang et al. (2015) \\
\hline Bbagt 1 & 5,7 & $\alpha$-glucose transporter & Reduced virulence, germination and conidial yield & Wang et al. (2013c) \\
\hline Bbmpd & 5,6 & $\begin{array}{l}\text { Mannitol-1-phos- } \\
\text { phate dehydrogenase }\end{array}$ & $\begin{array}{l}\text { Reduced virulence, stress-tolerance and trehalase } \\
\text { expression }\end{array}$ & Wang et al. (2012) \\
\hline Bbmtd & 5,6 & $\begin{array}{l}\text { Mannitol dehydro- } \\
\text { genase }\end{array}$ & $\begin{array}{l}\text { Reduced virulence, stress-tolerance and trehalase } \\
\text { expression }\end{array}$ & Wang et al. (2012) \\
\hline $\mathrm{BbCreA}$ & $3,5,7$ & $\begin{array}{l}\text { Regulates CreA } \\
\text { activity }\end{array}$ & Reduced virulence, conidiation and nutrient uptake & Luo et al. (2014) \\
\hline Bbcsa1 & 5 & $\begin{array}{l}\text { Calcium sensor, } \\
\text { acidification regulator }\end{array}$ & Reduced extracellular acidification & Fan et al. (2012b) \\
\hline Bbbeas & 5 & Beauvericin toxin & Reduced virulence and beauvericin production & Xu et al. (2008) \\
\hline Bbbsls & 5 & Bassianolide toxin & Reduced virulence and bassianolide production & Xu et al. (2009) \\
\hline $\begin{array}{l}\text { (geneID } \\
\text { not known) }\end{array}$ & 5 & Bassiacridin & (Purified protein showed activity specific to locusts) & $\begin{array}{l}\text { Quesada-Moraga and } \\
\text { Vey (2004) }\end{array}$ \\
\hline ktr1 & 6 & $\underset{\text { ferase }}{\alpha-1,2 \text {-mannosyltrans- }}$ & Reduced conidial thermotolerance & Wang et al. (2014b) \\
\hline ktr2, ktr4 & 6,7 & $\begin{array}{l}\alpha-1,2 \text {-mannosyltrans- } \\
\text { ferase }\end{array}$ & Reduced conidiation and stress-tolerance & Wang et al. (2014b) \\
\hline $\operatorname{ras} 1, \operatorname{ras} 2, \operatorname{ras} 3$ & $2,6,7$ & $\begin{array}{l}\text { GTPase, signal } \\
\text { transduction }\end{array}$ & $\begin{array}{l}\text { Reduced virulence, germination, conidiation, } \\
\text { stress-tolerance }\end{array}$ & $\begin{array}{l}\text { Xie et al., } 2013 \text { and } \\
\text { Guan et al., } 2015\end{array}$ \\
\hline Bbac & 6,7 & cAMP signaling & Reduced virulence, conidiation and stress-tolerance & Wang et al. (2014a) \\
\hline $\operatorname{cdc} 14$ & $4,6,7$ & Cytokinesis & $\begin{array}{l}\text { Reduced blastospore production, conidial yield and } \\
\text { stress-tolerance }\end{array}$ & Wang et al. (2013a) \\
\hline Bbmsn2 & $3,6,7$ & Transcription factor & Reduced conidial yield and stress-tolerance & Liu et al. (2013) \\
\hline
\end{tabular}




\begin{tabular}{|c|c|c|c|c|}
\hline $\operatorname{sod} 1-5$ & 6 & $\begin{array}{l}\text { Superoxide } \\
\text { dismutases }\end{array}$ & Reduced stress-tolerance & Li et al. (2014) \\
\hline vcx $1 \mathrm{~A}-\mathrm{E}$ & 6 & $\begin{array}{l}\text { Vacuolar calcium } \\
\text { exchangers }\end{array}$ & Reduced virulence and stress-tolerance & Hu et al. (2014) \\
\hline catA-D, catP & 6 & Catalase & Reduced virulence, stress-tolerance & Wang et al. (2013d) \\
\hline $\operatorname{trx} 1-6$ & 6 & $\begin{array}{l}\text { Thioredoxin antiox- } \\
\text { idants }\end{array}$ & $\begin{array}{l}\text { Reduced virulence, germination, conidiation and } \\
\text { stress-tolerance }\end{array}$ & Zhang et al. (2015) \\
\hline $\begin{array}{l}\text { Mdr1, Mrp1, } \\
\text { Pdr1, Pdr2, Pdr5 }\end{array}$ & 6 & $\mathrm{ABC}$ transporters & Reduced stress-tolerance & Song et al. (2013) \\
\hline $\begin{array}{l}\mathrm{CnA} 1, \mathrm{CnA} 2 \\
\mathrm{CnB}, \mathrm{Crz} 1\end{array}$ & 6,7 & $\begin{array}{l}\text { Calcineuring-mediat- } \\
\text { ed phosphatase }\end{array}$ & Reduced virulence, stress-tolerance and conidiation & Li et al. (2015) \\
\hline Bbrgs1 & 7 & G-protein signaling & $\begin{array}{l}\text { Reduced conidial yield, thermotolerance and } \\
\text { viability }\end{array}$ & Fang et al. (2008) \\
\hline BbGPCR3 & 4,7 & $\begin{array}{l}\text { G-protein coupled } \\
\text { receptor }\end{array}$ & $\begin{array}{c}\text { Reduced conidiation, virulence and transition to } \\
\text { blastospores }\end{array}$ & Ying et al. (2013) \\
\hline Bbslt2 & 7 & MAP kinase & $\begin{array}{l}\text { Reduced conidial yield and viability, cell growth and } \\
\text { cell wall sensitivity }\end{array}$ & Luo et al. (2012) \\
\hline BbmtrA & 7 & $\begin{array}{l}\text { Putative } \\
\text { methyltransferase }\end{array}$ & $\begin{array}{l}\text { Reduced germination, growth, conidiation and } \\
\text { virulence }\end{array}$ & Qin et al. (2014) \\
\hline Bbgas 1 & 2,7 & $\begin{array}{l}\text { Cell-wall anchored } \\
\text { enzyme }\end{array}$ & Reduced virulence and thermotolerance & Zhang et al. (2011a) \\
\hline Bbjen1 & 7 & $\begin{array}{l}\text { Carboxylate } \\
\text { transporter }\end{array}$ & Reduced virulence, increased conidial yield & Jin et al. (2010) \\
\hline Bbpmr1 & 7 & ATPase, signaling & Reduced conidiation & Wang et al. (2013b) \\
\hline
\end{tabular}

A cytochrome P450 gene, Bbcyp52x1, was shown to function in enzymatic breakdown of lipids in the waxy layer. Conidia of a $\triangle B b c y p 52 \times 1$ knock-out mutant showed impaired germination on grasshopper wings. This knock-out mutant also showed reduced virulence on the Greater Wax moth Galleria mellonella upon topical application of conidia, though not upon intrahemocoel injection. Similar to Magas1 (step 2) this suggests that the Bbcyp52x1 gene promotes virulence specifically via its function in cuticle penetration (Zhang et al., 2012). B. bassiana is known to secrete lipases (Leopold and Samšiňáková, 1970), which are thought to play a role in degradation of the waxy layer. However, a study by Feng (1998) indicated that the total lipase activity of various strains had little correlation to their virulence.

As indicated above, proteins are the main component of the insect cuticle and assays with cuticles from which proteins had been removed indicated that proteins are the most important nutrient source for germination and hyphal growth on the cuticle (Bidochka and Khachatourians, 1992). Therefore, not surprisingly, extracellular proteases are important for cuticle breakdown by entomopathogenic fungi. Specifically in B. bassiana, subtilisin-like ( $\operatorname{Pr} 1)$ and trypsin-like $(\operatorname{Pr} 2)$ cuticle-degrading proteases were shown to be involved, as these genes were induced by the presence of cuticle compounds of different insects (Joshi et al., 1995). The $\operatorname{Pr} 1$ gene $c d e p 1$ also showed involvement in cuticle breakdown in B. bassiana (Fang et al., 2008). 


\section{Chapter 2}

The insect chitin framework can be degraded by secreted chitinases. Several B. bassiana chitinases were studied with respect to their function in this process. Fang et al. (2005) experimentally supported the involvement of a B. bassiana chitinase in virulence on aphids, Myzus persicae. Overexpression of chitin gene Bbchit1 increased virulence, both by increasing the infection efficiency and accelerating the infection (Fang et al., 2005). Recently, Liu et al. (2013) reported that a $\triangle B b m s n 2$ knock-out mutant displayed lower transcript levels of the three chitinase genes Chi1, Chi2 and ChsA2, and showed reduced virulence (Liu et al., 2013).

A study into the protein kinase gene Bbsnf1 showed a reduction of virulence in the $\triangle B b s n f 1$ knock-out mutant, and the reduction was more pronounced upon topical application then upon intrahemocoel injection. This suggests a role for this regulator in cuticle penetration, though the exact mode of action of BbSNF1 is currently not known (Wang et al., 2014c).

\subsection{Transition to blastospore growth}

Hyphae that have successfully grown through the cuticle and reached the haemocoel can switch to a yeast-like growth mode. The fungus forms single-cell hyphal bodies called blastospores (in vivo blastospores), which have a unique brush-like outermost structure (Wanchoo et al., 2009). This is a cryptic growth form, and this unique structure is thought to help blastospores avoid detection by the host immune system. Blastospores proliferate via budding and use the circulating haemolymph as a 'vehicle' for colonization of the host as well as a source of nutrition (Lewis et al., 2009).

The identities of genes specifically regulating the developmental transition from hyphal growth to blastospores remains unknown in B. bassiana, though certain regulatory genes of many biological processes were shown to be involved in blastospore development. For example, analysis of the role of the weel gene and the $c d c 25$ gene (which both regulate the cell cycle-required cyclindependent kinase $1 \mathrm{Cdk1}$ ), showed that blastospore size was reduced in the knock-out mutants in these genes (Qiu et al., 2015). The forkhead transcription factor Fkh2 is also involved in the regulation of the cell cycle, and the deletion of $F k h 2$ produced smaller blastospores as well, but twice as many blastospores compared the wild-type (Wang et al., 2015). Moreover, disruption of the Bbsnf1 gene, a protein kinase involved in fungal development, led to lower blastospore yield (Wang et al., 2014c).

\subsection{Colonization and killing of the host}

Different virulence determinants play a role during host colonization, both for growth in the insect and to mediate the death of the host. During infection, the insect body is the source 
of all nutrition for the fungus, and especially the disaccharide trehalose, which is the most abundant carbohydrate in the insect hemolymph. The fungus either secretes trehalase to hydrolyze the circulating trehalose and take up the resulting glucose, or imports trehalose to process it intracellularly (Xia et al., 2002). The $\alpha$-glucoside transporter gene Bbagt1 was shown to be necessary for trehalose uptake, and its deletion resulted in reduced virulence (Wang et al., 2013c). In addition to trehalose utilization, it is likely that virulence is correlated with the metabolism and biosynthesis of other sugars. One of the few detailed studies on this topic shows that the mannitol dehydrogenase genes Bbmpd and Bbmtd, involved in mannitol biosynthesis, are also required for full virulence. Interestingly, the knock-out mutant strains of each gene also showed a decreased trehalase expression (Wang et al., 2012). Another example is the $B b C r e A$ regulator that plays an important role in nutrient utilization. The $\triangle B b C r e A$ knock-out mutant showed a reduced growth in carbohydrate substrates, improper assimilation of some amino acids as well as reduced virulence and conidiation (Luo et al., 2014).

During infection, the fungal cells encounter different $\mathrm{pH}$ values, depending on the environment. The $\mathrm{pH}$ inside a host varies between insect species and tissues, and it changes with age (Schultz and Lechowicz, 1986). Secreted enzymes have a $\mathrm{pH}$ at which their activity is optimal, and therefore gene expression must be adapted to the ambient $\mathrm{pH}$. In Metarhizium anisopliae, several genes encoding cuticle degrading enzymes showed highest transcript levels around their optimal $\mathrm{pH}$ (St Leger et al., 1998). The $\mathrm{pH}$-dependent regulation of expression is mediated by response elements in the genome. The transcription factor $B b p a c C$ is one such element that enables $B$. bassiana to adapt its gene expression to ambient $\mathrm{pH}$ (Peñalva et al., 2008). Alkaline $\mathrm{pH}$ strongly induces $B b p a c C$, leading to upregulation of alkaline-expressed genes and repression of acidexpressed genes (Zhou et al., 2014).

The ambient $\mathrm{pH}$ can also be regulated by secretion of compounds that acidify or alkalify the surrounding microenvironment (St Leger et al., 1999). Progress of the infection can be stimulated by acidification of the host tissue and haemolymph. To this end the fungus secretes acidic metabolites such as oxalic acid (Bidochka and Khachatourians, 1991). A factor involved in acidification is the $B b c s a 1$ gene (calcium sensor and acidification regulator). Deletion of the Bbcsal gene resulted in reduced acidification accompanied by reduced virulence (Fan et al., 2012b).

In addition to acidifying compounds, the fungus can secrete toxic metabolites to speed up the death of the host. B. bassiana produces beauvericin, which has insecticidal, antifungal and cytotoxic properties. Beauvericin synthase (Bbbeas) was found to be important, though not 


\section{Chapter 2}

essential for virulence, as different insect hosts infected with the $\triangle$ Bbbeas knock-out mutant showed significantly reduced mortality rates (Xu et al., 2008). The cytotoxic cyclodepsipeptide bassianolide synthase $(B b b s l s)$ has been detected in cadavers of silkworms killed by B. bassiana, suggesting that production of this toxin coincides with infection and insect death. $\Delta B b b s l s$ knockout mutant strains showed decreased virulence on different hosts (Xu et al., 2009). Another study showed secretion of the toxin bassiacridin, which increases virulence specifically on locusts (Quesada-Moraga and Vey, 2004).

\subsection{Interactions with the insect immune system}

From cuticle adhesion onwards, the fungus has to survive the insect immune responses and including defense mechanisms such as toxins, protease inhibitors or other infection-inhibiting compounds that can be present in the cuticle (for a review on this topic, see Ortiz-Urquiza and Keyhani (2013)). The fungus must either evade or overcome the host defense mechanisms (e.g. by secreting compounds that modulate the insect response or by morphological structures in blastospores). Details are scarce on the genetic regulation of the responses of entomopathogenic fungi to the host defence mechanisms.

The insect innate immune responses are regulated by the Toll and Imd (Immune deficiency) pathways. The Toll signalling pathway is activated by the ligand Spätzle, which can be activated via serine protease cascades by microbial compounds such as fungal $\beta$-1,3-glucans (Valanne et al., 2011). The insect immune system can be divided in a humoral and a cellular immune response. A humoral response includes the release of a range of anti-microbial peptides (AMPs) into the haemolymph (Tanji et al., 2007), the release of reactive oxygen species (ROS) and a complex enzymatic cascade that regulate clotting or melanization (Jiravanichpaisal et al., 2006). Activation of a melanization response leads to the deposition of melanin on the surface of parasites or wound surface. This results in the release of ROS and encapsulation of the fungal cells (Pham et al., 2007 and Yassine et al., 2012). Consequently, mortality of Anopheles gambiae larvae infected with the mold Lagenidium giganteum was reduced by melanization of germ tubes (Golkar et al., 1993).

In contrast, the cellular response is governed by different types of haemocytes, which can encapsulate, phagocytize and kill invading cells (Pham et al., 2007). Blastospores of B. bassiana phagocytized by Spodoptera exigua granulocytes were found to escape by growing a germ tube out of the encapsulation, where they started budding to form new blastospores (Hung et al., 1993). In addition, haemocyte production in infected larvae was reduced (caused by immunosuppressive compounds) and the haemocytes that were produced showed reduced efficacy in encapsulating fungal cells (Hung and Boucias, 1992). It is unknown which fungal genes contribute to 
overcoming immune responses in the insect host, but it is tempting to speculate the involvement of effector proteins (small secreted cysteine-rich proteins) that are able to suppress the immune response, as extensively studied in plant pathogenic fungi (Stergiopoulos and de Wit, 2009). Next to the escape reaction, hyphal bodies seem able to simply evade recognition by the absence of antigenic compounds on the cell surface, such as chitin and galactomannan (Pendland et al., 1993). In M. anisopliae, the MCL1 protein was found to mask compounds on the cell surface and thereby enables hyphal bodies to avoid detection by haemocytes in the insect haemolymph. The hydrophilic properties and negative charge of MCL1 proteins reduce the adhesion of hyphal bodies to haemocytes (Wang and St Leger, 2006). No homolog of MCL1 has been detected in the B. bassiana genome and it is unknown whether a different protein fulfils an analogous function to the MCL1 protein.

Insect hosts can increase haemolymph ROS concentrations in response to infection. This increases survival after infection, as shown in An. gambiae infected with bacteria or Plasmodium (MolinaCruz et al., 2008). The host employs enzymes to protect itself against ROS, among which are catalases and thioredoxin peroxidases to detoxify $\mathrm{H}_{2} \mathrm{O}_{2}$, and superoxide dismutases (SODs) to detoxify $\mathrm{O}_{2}$ - superoxide (Molina-Cruz et al., 2008). For successful infection, the invading fungus must likewise protect itself against exposure to ROS and concomitant cellular stress. A number of genes were found to be involved in ROS protection and stress tolerance, which are not only relevant for infection but also for other life stages of the fungus. Those genes are mostly regulators that determine virulence by affecting multiple interconnected downstream processes, among which are conidiation, multi-stress responses, germination, and growth.

As examples of such regulators, the genes Ras 1 , Ras 2 and Ras3 influence virulence by affecting multi-stress tolerance, germination and growth, among others (Guan et al., 2015 and Xie et al., 2013). The adenylate cyclase gene Bbac, operating in cAMP signalling, was also found to contribute to virulence, by regulating multi-stress responses and conidiation. The $\triangle B b a c$ knockout mutant was highly sensitive to stress by high osmolarity, oxidation, cell wall damage and different chemicals. Bbac, similar to other regulators, seems to improve tolerance to oxidation by positively regulating antioxidation (Wang et al., 2014a). All these genes have regulatory functions and more detailed studies are required to reveal their downstream target genes and specific roles.

Additional interesting examples of virulence genes functioning in stress responses are calcineurin subunits $C n A 1, C n A 2$ and $C n B$ and their downstream transcription factor $C r z 1$ (Li et al., 2015), the required for cytokinesis gene $C d c 14$ (Wang et al., 2013a), the zinc finger transcription factor Bbmsn2 (Liu et al., 2013), superoxide dismutases sod1-5 (Li et al., 2014), vacuolar calcium 


\section{Chapter 2}

exchangers $v c x 1 A-E$ (Hu et al., 2014), catalases $\operatorname{cat} A-D$ and $\operatorname{cat} P$ (Wang et al., 2013d), the $\alpha-1,2-$ mannosyltransferases Ktr1, Ktr2 and Ktr4 (Wang et al., 2014b), the thioredoxin antioxidants trx1-6 (Zhang et al., 2015), and the ATP-binding cassette (ABC) transporters Mdr1, Mrp1, Pdr1, Pdr2, and Pdr5 (Song et al., 2013). For a more specific review on factors contributing to (environmental) stress tolerance in entomopathogenic fungi, see Ortiz-Urquiza and Keyhani (2014).

\subsection{Hyphal extrusion and conidiation}

When the nutrients in the host are depleted and the host has died, the fungus must grow out of the insect to produce and disperse its conidia (Fig. 1B). Blastospores still circulating in the haemocoel must revert to growth as hyphae, which have to breach the cuticle again - this time from the inside out - to enable hyphal extrusion followed by conidiation on the host surface. It is likely that the same factors are involved here as in breaching the cuticle from the outside, as discussed in infection step 2 .

In the previously discussed $\triangle B b m p k 1$ knock-out mutant, the expression of several protease and chitinase genes was unaltered but the fungus failed to breach the cuticle from the inside and did not proceed to conidiation. This indicates that $B b M P K 1$ has a vital role not only for appressorium formation and conidiation but also is crucial for penetration of the insect cuticle both from the outside inwards and from the inside outwards (Zhang et al., 2010). Similar indications that the same factors are involved in inward and outward cuticle penetration were made in Metarhizium acridum for the MaPls1 gene (Luo et al., 2013). Hyphae that have escaped the insect body can proceed to the production of conidia. This process is induced by sensing of favourable environmental conditions via $G$ protein-mediated signalling in filamentous fungi. Regulators of G-protein signalling (RGS) are also involved in other processes, such as germination, stress tolerance and secretion of toxins (Fang et al., 2008). The RGS gene Bbrgs 1 was shown to be involved in conidiation, as gene disruption reduced conidial yield, as well as conidial viability and thermotolerance. Remarkably, virulence was unaffected both upon topical application and intrahaemocoel injection (Fang et al., 2008). Involvement of RGS genes in conidiation of Metarhizium has also been confirmed (Fang et al., 2007). In addition, a G-protein coupled receptor (GPCR) BbGPCR3 was shown to be involved in conidiation, nutrient sensing and stress response. A $\triangle B b G P C R 3$ knock-out mutant showed reduced conidiation and transition to blastospores, and a decreased virulence in topical applications (Ying et al., 2013).

Genes specifically acting in conidiation are not well studied in B. bassiana, but the involvement of various regulators has been reported. Effects on conidiation often manifested in a reduction of 
conidial yield and viability, and/or altered morphology of conidia-bearing aerial hyphae. Studies on regulatory genes indicate that conidiation is connected with other developmental processes. Reduced conidiation, along with disturbance of other developmental processes, was observed upon disruption of the MAP kinase Bbslt2 (Luo et al., 2012) and the previously discussed Bbmpk1 (Zhang et al., 2010) and Bbhog1 (Zhang et al., 2009). The cell wall anchored enzyme Bbgas 1 proved to be involved in conidial thermotolerance and virulence. Conidia of the $\triangle B b g a s 1$ knock-out mutant showed reduced virulence to $G$. mellonella upon both topical application and intrahemocoel injection, and heat-shock treated conidia showed decreased ability to germinate (Zhang et al., 2011a). The putative methyltransferase BbmtrA was shown to be involved in conidial viability, fungal growth and virulence (Qin et al., 2014). The carboxylate transporter $B b j e n 1$ also is involved in conidiation and virulence, as the $\triangle B b j e n 1$ knock-out mutant displayed reduced virulence but increased conidial yield (Jin et al., 2010).

More regulators are known to affect conidiation. Some examples are the previously discussed Bbac (Wang et al., 2014a) and Bbmsn2 (Liu et al., 2013), a knock-out mutant of the gene $A 43$ had a reduced conidial yield (Wu et al., 2008) and the P-type Ca2+-ATPase Bbpmr1 regulates conidiation by functioning in different signalling cascades (Wang et al., 2013b).

Studies on regulators make it clear that different developmental processes are connected via signaling pathways. One study established the role of the $O h m m 1$ gene, which was downregulated in $\triangle B b h o g 1$. A $\triangle O h m m 1$ knock-out mutant showed increased virulence and oxidative stress tolerance, and reduced conidiation (He et al., 2015). Additional studies that focus on the downstream targets of regulators are required to reveal the roles of specific genes and to obtain a more detailed understanding of conidiation and its impact on virulence in B. bassiana.

\section{Conclusions and perspective}

Infection of an insect by B. bassiana comprises numerous complex developmental transitions, which require intricate genetic regulation. Specific roles of a number of regulatory genes in B. bassiana have been established, while the roles of others have yet to be unraveled. A better understanding of the network of genes that contribute to virulence could ideally be used for selecting strains with the most virulent genotype. Insight in virulence genes (see Table 1 for an overview) can contribute to improving the efficiency of biocontrol products, which can serve as alternatives to chemical pesticides to prevent disease spreading and crop loss. Although, substantial knowledge has been gained on genes influencing virulence, the distinction between genes specifically involved in entomopathogenic virulence versus general biological processes is insufficiently addressed. Previous studies have often focused on the role of global regulatory genes 


\section{Chapter 2}

that control the expression of many downstream genes. Knocking-out a regulatory gene results in pleiotropic loss of fitness, and inevitably culminates in reduction of virulence on insects. Therefore the focus on dissecting the factors uniquely contributing to virulence on insects is a necessity. More specific virulence factors could be discovered via comparative gene expression analyses of B. bassiana strains with marked differences in virulence (Valero-Jiménez et al., 2014). Another strategy may be the use of an experimental evolution approach with the aim to identify genes and mechanisms underpinning virulence (unpublished data). Experimental evolution in combination with whole genome sequencing is a powerful tool since it could link genotype and phenotype (e.g. increased virulence, (Long et al., 2015)). An important additional aspect is that all conducted experiments should be based on a common methodology that comprises using similar target insects and a natural infection method with a constant concentration of spores and same time of exposure. Once specific virulence factors are identified, it will be important to explore the natural variation (in nucleotide sequence and expression of these genes) in the fungal population. It is also highly relevant to anticipate how insects can undergo adaptation to evade the action of fungal virulence factors and thereby develop resistance to the biocontrol agent.

Ultimately, these approaches could collectively increase the potential of biopesticides, through strain improvement in B. bassiana. It is also worth noting that other approaches that increase the potential applications of $B$. bassiana as a biocontrol agent have been suggested. For instance, transgenic strategies have been employed to express endogenous B. bassiana chitinases (Fang et al., 2005), or heterologous toxins (Lu et al., 2008), or to express host molecules (Fan et al., 2012a), all in order to shift the balance of the interaction in favour of the pathogen, and thereby increasing efficacy in virulence. However, the use of genetically modified organisms has numerous hurdles that limit their applications in the field. 


\section{References}

Bergvinson, D., García-Lara, S., 2004. Genetic approaches to reducing losses of stored grain to insects and diseases. Curr Opin Plant Biol 7, 480-485. doi:10.1016/j. pbi.2004.05.001

Bhatt, S., Gething, P.W., Brady, O.J., Messina, J.P., Farlow, A.W., Moyes, C.L., Drake, J.M., Brownstein, J.S., Hoen, A.G., Sankoh, O., Myers, M.F., George, D.B., Jaenisch, T., Wint, G.R.W., Simmons, C.P., Scott, T.W., Farrar, J.J., Hay, S.I., 2013. The global distribution and burden of dengue. Nature 496, 504-7. doi:10.1038/ nature 12060

Bidochka, M., Kamp, A., de Croos, J.N.A., 2000. Insect Pathogenic Fungi: From Genes to Populations, in: Kronstad, J.W. (Ed.), Fungal Pathology. Springer Netherlands, pp. 171-193. doi:10.1007/978-94-0159546-9_7

Bidochka, M.J., Khachatourians, G.G., 1992. Growth of the Entomopathogenic Fungus Beauveria bassiana on Cuticular Components From the Migratory Grasshopper, Melanoplus sanguinipes. J Invertebr Pathol 59, 165-173. doi:10.1016/0022-2011(92)90028-3

Bidochka, M.J., Khachatourians, G.G., 1991. The implication of metabolic acids produced by Beauveria bassiana in pathogenesis of the migratory grasshopper, Melanoplus sanguinipes. J Invertebr Pathol 58, 106-117. doi:10.1016/0022-2011(91)90168-P

Blanford, S., Chan, B.H.K., Jenkins, N., Sim, D., Turner, R.J., Read, A.F., Thomas, M.B., 2005. Fungal pathogen reduces potential for malaria transmission. Science 308 , 1638-41. doi:10.1126/science. 1108423

Boomsma, J.J., Jensen, A.B., Meyling, N.V., Eilenberg, J., 2014. Evolutionary interaction networks of insect pathogenic fungi. Annu Rev Entomol 59, 467-485. doi:10.1146/annurev-ento-011613-162054

Boucias, D.G., Pendland, J.C., Latge, J.P., 1988. Nonspecific factors involved in attachment of entomopathogenic deuteromycetes to host insect cuticle. Appl Environ Microbiol 54, 1795-1805.

Brownbridge, M., Costa, S., Jaronski, S.T., 2001. Effects of in vitro passage of Beauveria bassiana on virulence to Bemisia argentifolii. J Invertebr Pathol 77, 280-283. doi:10.1006/jipa.2001.5020

Cao, Y., Zhu, X., Jiao, R., Xia, Y., 2012. The Magas1 gene is involved in pathogenesis by affecting penetration in Metarhizium acridum. J Microbiol Biotechnol 22, 889893. doi:10.4014/jmb.1111.11055

Charnley, A.K., St. Leger, R.J., 1991. The Role of CuticleDegrading Enzymes in Fungal Pathogenesis in Insects, in: Cole, G., Hoch, H. (Eds.), The Fungal Spore and Disease Initiation in Plants and Animals. Springer US, pp. 267-286. doi:10.1007/978-1-4899-2635-7_12

Clarkson, J.M., Charnley, A.K., 1996. New insights into the mechanisms of fungal pathogenesis in insects. Trends Microbiol 4, 197-203.

Cruz, L.P., Gaitan, A.L., Gongora, C.E., 2006. Exploiting the genetic diversity of Beauveria bassiana for improving the biological control of the coffee berry borer through the use of strain mixtures. Appl Microbiol Biotechnol 71, 918-926. doi:10.1007/s00253-005-0218-0

de Faria, M.R., Wraight, S.P., 2007. Mycoinsecticides and Mycoacaricides: A comprehensive list with worldwide coverage and international classification of formulation types. Biol Control 43, 237-256. doi:10.1016/j. biocontrol.2007.08.001

Fan, Y., Borovsky, D., Hawkings, C., Ortiz-Urquiza, A., Keyhani, N., 2012a. Exploiting host molecules to augment mycoinsecticide virulence. Nat Biotechnol 30, 35-7. doi:10.1038/nbt.2080

Fan, Y., Ortiz-Urquiza, A., Kudia, R. a., Keyhani, N.O., 2012b. A fungal homologue of neuronal calcium sensor-1, Bbcsa1, regulates extracellular acidification and contributes to virulence in the entomopathogenic fungus Beauveria bassiana. Microbiol (United Kingdom) 158, 1843-1851. doi:10.1099/mic.0.058867-0

Fang, W., Leng, B., Xiao, Y., Jin, K., Ma, J., Fan, Y., Feng, J., Yang, X., Zhang, Y., Pei, Y., 2005. Cloning of Beauveria bassiana chitinase gene Bbchit1 and its application to improve fungal strain virulence. Appl Environ Microbiol 71, 363-370. doi:10.1128/AEM.71.1.363-370.2005

Fang, W., Pei, Y., Bidochka, M.J., 2007. A regulator of a $\mathrm{G}$ protein signalling (RGS) gene, cag8, from the insect-pathogenic fungus Metarhizium anisopliae is involved in conidiation virulence and hydrophobin synthesis. Microbiology 153, 1017-1025. doi:10.1099/ mic.0.2006/002105-0

Fang, W., Scully, L.R., Zhang, L., Pei, Y., Bidochka, M.J., 2008. Implication of a regulator of $G$ protein signalling (BbRGS1) in conidiation and conidial thermotolerance of the insect pathogenic fungus Beauveria bassiana. FEMS Microbiol Lett 279, 146-156. doi:10.1111/ j.1574-6968.2007.00978.x

Golkar, L., LeBrun, R.A., Ohayon, H., Gounon, P., Papierok, B., Brey, P.T., 1993. Variation of larval susceptibility to Lagenidium giganteum in three mosquito species. J Invertebr Pathol. doi:10.1006/jipa.1993.1066

Guan, Y., Wang, D.-Y., Ying, S.-H., Feng, M.-G., 2015. A novel Ras GTPase (Ras3) regulates conidiation, multistress tolerance and virulence by acting upstream of Hog1 signaling pathway in Beauveria bassiana. Fungal Genet Biol 82, 85-94. doi:10.1016/j.fgb.2015.07.002

He, Z., Zhang, S., Keyhani, N.O., Song, Y., Huang, S., Pei, Y., Zhang, Y., 2015. A novel mitochondrial membrane 


\section{Chapter 2}

protein, Ohmm, limits fungal oxidative stress resistance and virulence in the insect fungal pathogen Beauveria bassiana. Environ Microbiol. doi:10.1111/14622920.12713

Holder, D.J., Keyhani, N.O., 2005. Adhesion of the entomopathogenic fungus Beauveria (Cordyceps) bassiana to substrata. Appl Environ Microbiol 71, 5260-5266. doi:10.1128/AEM.71.9.5260

Hu, Y., Wang, J., Ying, S.-H., Feng, M.-G., 2014. Five vacuolar $\mathrm{Ca} 2+$ exchangers play different roles in calcineurin-dependent $\mathrm{Ca} 2+/ \mathrm{Mn} 2+$ tolerance, multistress responses and virulence of a filamentous entomopathogen. Fungal Genet Biol 73, 12-19. doi:10.1016/j.fgb.2014.09.005

Hung, S.-Y., Boucias, D.G., 1992. Influence of Beauveria bassiana on the Cellular Defense Response of the Beet Armyworm, Spodopfera exigua. J Invertebr Pathol 60, $152-158$.

Hung, S.-Y., Boucias, D.G., Vey, A.J., 1993. Effect of Beauveria bassiana and Candida albicans on the cellular defense response of Spodoptera exigua. J Invertebr Pathol. doi:S0022-2011(83)71032-3 [pii] \r10.1006/ jipa.1993.1032

Jin, K., Zhang, Y., Fang, W., Luo, Z., Zhou, Y., Pei, Y., 2010. Carboxylate transporter gene JEN1 from the entomopathogenic fungus Beauveria bassiana is involved in conidiation and virulence. Appl Environ Microbiol 76, 254-263. doi:10.1128/AEM.00882-09

Jiravanichpaisal, P., Lee, B.L., Söderhäll, K., 2006. Cellmediated immunity in arthropods: Hematopoiesis, coagulation, melanization and opsonization. Immunobiology 211, 213-236. doi:10.1016/j. imbio.2005.10.015

Jones, W.E., Grace, J.K., Tamashiro, M., 1996. Virulence of Seven Isolates of Beauveria bassiana and Metarhizium anisopliae to Coptotermes formosanus (Isoptera: Rhinoterrnitidae). Environ Entomol 25, 481-487.

Joshi, L., St Leger, R.J., Bidochka, M.J., 1995. Cloning of a cuticle-degrading protease from the entomopathogenic fungus, Beauveria bassiana. FEMS Microbiol Lett 125, 211-217. doi:10.1016/0378-1097(94)00500-Q

Kirkland, B.H., Westwood, G.S., Keyhani, N.O., 2004. Pathogenicity of entomopathogenic fungi Beauveria bassiana and Metarhizium anisopliae to Ixodidae tick species Dermacentor variabilis, Rhipicephalus sanguineus, and Ixodes scapularis. J Med Entomol 41, 705-711. doi:10.1603/0022-2585-41.4.705

Leopold, J., Samšiňáková, A., 1970. Quantitative estimation of chitinase and several other enzymes in the fungus Beauveria bassiana. J Invertebr Pathol 15, 34-42. doi:10.1016/0022-2011(70)90095-9

Lewis, M.W., Robalino, I. V., Keyhani, N.O., 2009.
Uptake of the fluorescent probe FM4-64 by hyphae and haemolymph-derived in vivo hyphal bodies of the entomopathogenic fungus Beauveria bassiana. Microbiology 155, 3110-3120. doi:10.1099/ mic.0.029165-0

Li, F., Shi, H.-Q., Ying, S.-H., Feng, M.-G., 2014. Distinct contributions of one $\mathrm{Fe}$ - and two $\mathrm{Cu} / \mathrm{Zn}$-cofactored superoxide dismutases to antioxidation, UV tolerance and virulence of Beauveria bassiana. Fungal Genet Biol. doi:10.1016/j.fgb.2014.09.006

Li, F., Wang, Z.-L., Zhang, L.-B., Ying, S.-H., Feng, M.G., 2015. The role of three calcineurin subunits and a related transcription factor (Crz1) in conidiation, multistress tolerance and virulence in Beauveria bassiana. Appl Microbiol Biotechnol 99, 827-840. doi:10.1007/ s00253-014-6124-6

Liu, Q., Ying, S.-H., Li, J.-G., Tian, C.-G., Feng, M.-G., 2013. Insight into the transcriptional regulation of Msn2 required for conidiation, multi-stress responses and virulence of two entomopathogenic fungi. Fungal Genet Biol 54, 42-51. doi:10.1016/j.fgb.2013.02.008

Long, A., Liti, G., Luptak, A., Tenaillon, O., 2015. Elucidating the molecular architecture of adaptation via evolve and resequence experiments. Nat Publ Gr 16, 567-582. doi: $10.1038 / \operatorname{nrg} 3937$

Lord, J.C., Howard, R.W., 2004. A proposed role for the cuticular fatty amides of Liposcelis bostrychophila (Psocoptera: Liposcelidae) in preventing adhesion of entomopathogenic fungi with dry-conidia. Mycopathologia 158, 211-217. doi:10.1023/ B:MYCO.0000041837.29478.78

Lu, D., Pava-Ripoll, M., Li, Z., Wang, C., 2008. Insecticidal evaluation of Beauveria bassiana engineered to express a scorpion neurotoxin and a cuticle degrading protease. Appl Microbiol Biotechnol 81, 515-22. doi:10.1007/s00253-008-1695-8

Luo, S., He, M., Cao, Y., Xia, Y., 2013. The tetraspanin gene MaPls1 contributes to virulence by affecting germination, appressorial function and enzymes for cuticle degradation in the entomopathogenic fungus, Metarhizium acridum. Environ Microbiol 15, 2966 2979. doi:10.1111/1462-2920.12166

Luo, X., Keyhani, N.O., Yu, X., He, Z., Luo, Z., Pei, Y., Zhang, Y., 2012. The MAP kinase Bbslt2 controls growth, conidiation, cell wall integrity, and virulence in the insect pathogenic fungus Beauveria bassiana. Fungal Genet Biol 49, 544-55. doi:10.1016/j.fgb.2012.05.002

Luo, Z., Qin, Y., Pei, Y., Keyhani, N.O., 2014. Ablation of the creA regulator results in amino acid toxicity, temperature sensitivity, pleiotropic effects on cellular development and loss of virulence in the filamentous fungus Beauveria bassiana. Environ Microbiol 16, 1122 36. doi:10.1111/1462-2920.12352 
Molina-Cruz, A., DeJong, R.J., Charles, B., Gupta, L., Kumar, S., Jaramillo-Gutierrez, G., Barillas-Mury, C., 2008. Reactive oxygen species modulate Anopheles gambiae immunity against bacteria and Plasmodium. J Biol Chem 283, 3217-3223. doi:10.1074/jbc. M705873200

Oerke, E.C., Dehne, H.W., 2004. Safeguarding production - Losses in major crops and the role of crop protection. Crop Prot 23, 275-285. doi:10.1016/j. cropro.2003.10.001

Oliveira, C.M., Auad, A.M., Mendes, S.M., Frizzas, M.R., 2014. Crop losses and the economic impact of insect pests on Brazilian agriculture. Crop Prot 56, 50-54. doi:10.1016/j.cropro.2013.10.022

Ortiz-Urquiza, A., Keyhani, N.O., 2014. Stress response signaling and virulence: insights from entomopathogenic fungi. Curr Genet. doi:10.1007/s00294-014-0439-9

Ortiz-Urquiza, A., Keyhani, N.O., 2013. Action on the surface: Entomopathogenic fungi versus the insect cuticle. Insects 4, 357-374. doi:10.3390/insects4030357

Pedrini, N., Ortiz-Urquiza, A., Huarte-Bonnet, C., Zhang, S., Keyhani, N.O., 2013. Targeting of insect epicuticular lipids by the entomopathogenic fungus Beauveria bassiana: hydrocarbon oxidation within the context of a host-pathogen interaction. Front Microbiol 4, 24. doi: $10.3389 /$ fmicb.2013.00024

Peñalva, M.A., Tilburn, J., Bignell, E., Arst, H.N., 2008. Ambient $\mathrm{pH}$ gene regulation in fungi: making connections. Trends Microbiol 16, 291-300. doi:10.1016/j.tim.2008.03.006

Pendland, J.C., Hung, S.Y., Boucias, D.G., 1993. Evasion of host defense by in vivo-produced protoplast-like cells of the insect mycopathogen Beauveria bassiana. J Bacteriol 175, 5962-5969.

Pham, L.N., Dionne, M.S., Shirasu-Hiza, M., Schneider, D.S., 2007. A specific primed immune response in Drosophila is dependent on phagocytes. PLoS Pathog 3. doi:10.1371/journal.ppat.0030026

Qin, Y., Ortiz-Urquiza, a., Keyhani, N.O., 2014. A putative methyltransferase, $m \operatorname{tr} A$, contributes to development, spore viability, protein secretion and virulence in the entomopathogenic fungus Beauveria bassiana. Microbiology 160, 2526-2537. doi:10.1099/ mic.0.078469-0

Qiu, L., Wang, J.-J., Ying, S.-H., Feng, M.-G., 2015. Wee1 and Cdc25 control morphogenesis, virulence and multistress tolerance of Beauveria bassiana by balancing cell cycle-required cyclin-dependent kinase 1 activity. Environ Microbiol 17, 1119-1133. doi:10.1111/14622920.12530

Quesada-Moraga, E., Vey, A., 2004. Bassiacridin, a protein toxic for locusts secreted by the entomopathogenic fungus Beauveria bassiana. Mycol Res 108, 441-452. doi: $10.1017 /$ S0953756204009724

Schultz, J.C., Lechowicz, M.J., 1986. Hostplant, larval age, and feeding behavior influence midgut $\mathrm{pH}$ in the gypsy moth (Lymantria dispar). Oecologia 71, 133-137. doi:10.1007/BF00377332

Shang, Y., Chen, P., Chen, Y., Lu, Y., Wang, C., 2015. MrSkn7 Controls Sporulation, Cell Wall Integrity, Autolysis, and Virulence in Metarhizium robertsii. Eukaryot Cell 14, 396-405. doi:10.1128/EC.00266-14

Song, T.-T., Zhao, J., Ying, S.-H., Feng, M.-G., 2013 Differential contributions of five $\mathrm{ABC}$ transporters to mutidrug resistance, antioxidion and virulence of Beauveria bassiana, an entomopathogenic fungus. PLoS One 8, e62179. doi:10.1371/journal.pone.0062179

St Leger, R.J., Joshi, L., Roberts, D., 1998. Ambient pH is a major determinant in the expression of cuticledegrading enzymes and hydrophobin by Metarhizium anisopliae. Appl Environ Microbiol 64, 709-713.

St Leger, R.J., Nelson, J.O., Screen, S.E., 1999. The entomopathogenic fungus Metarhizium anisopliae alters ambient $\mathrm{pH}$, allowing extracellular protease production and activity. Microbiology 145 ( Pt 1, 2691-2699.

Stergiopoulos, I., de Wit, P.J.G.M., 2009. Fungal effector proteins. Annu Rev Phytopathol 47, 233-263. doi:10.1146/annurev.phyto.112408.132637

Sung, J., Lee, J., Humber, R., Sung, G., Shrestha, B., 2006. Cordyceps bassiana and production of stromata in vitro showing Beauveria anamorph in Korea. Mycobiology 34, $1-6$.

Tanji, T., Hu, X., Weber, A.N.R., Ip, Y.T., 2007. Toll and IMD pathways synergistically activate an innate immune response in Drosophila melanogaster. Mol Cell Biol 27, 4578-4588. doi:10.1128/MCB.01814-06

Valanne, S., Wang, J.-H., Rämet, M., 2011. The Drosophila Toll signaling pathway. J Immunol 186, 649-56. doi:10.4049/jimmunol.1002302

Valero-Jiménez, C.A., Debets, A.J.M., van Kan, J.A.L., Schoustra, S.E., Takken, W., Zwaan, B.J., Koenraadt, C.J.M., 2014. Natural variation in virulence of the entomopathogenic fungus Beauveria bassiana against malaria mosquitoes. Malar J 13, 479. doi:10.1186/14752875-13-479

Wagner, B.L., Lewis, L.C., 2000. Colonization of Corn, Zea mays, by the Entomopathogenic Fungus Beauveria bassiana. Appl Environ Microbiol 66, 3468-3473. doi:10.1128/AEM.66.8.3468-3473.2000

Wanchoo, A., Lewis, M.W., Keyhani, N.O., 2009. Lectin mapping reveals stage-specific display of surface carbohydrates in vitro and haemolymph-derived cells of the entomopathogenic fungus Beauveria 


\section{Chapter 2}

bassiana. Microbiology 155, 3121-3133. doi:10.1099/ mic.0.029157-0

Wang, C., St Leger, R.J., 2006. A collagenous protective coat enables Metarhizium anisopliae to evade insect immune responses. Proc Natl Acad Sci USA 103, 66476652. doi: $10.1073 /$ pnas.0601951103

Wang, C., St. Leger, R.J., 2007a. The MAD1 adhesin of Metarhizium anisopliae links adhesion with blastospore production and virulence to insects, and the MAD2 adhesin enables attachment to plants. Eukaryot Cell 6, 808-816. doi:10.1128/EC.00409-06

Wang, C., St. Leger, R.J., 2007b. The Metarhizium anisopliae perilipin homolog MPL1 regulates lipid metabolism, appressorial turgor pressure, and virulence. J Biol Chem 282, 21110-21115. doi:10.1074/jbc. M609592200

Wang, J., Liu, J., Hu, Y., Ying, S.-H., Feng, M.-G., 2013 a. Cytokinesis-required $\mathrm{Cdc1} 4$ is a signaling hub of asexual development and multi-stress tolerance in Beauveria bassiana. Sci Rep 3, 3086. doi:10.1038/srep03086

Wang, J., Zhou, G., Ying, S.H., Feng, M.G., 2014. Adenylate cyclase orthologues in two filamentous entomopathogens contribute differentially to growth, conidiation, pathogenicity, and multistress responses. Fungal Biol 118, 422-431. doi:10.1016/j. funbio.2014.03.001

Wang, J., Zhou, G., Ying, S.-H., Feng, M.-G., 2013b. P-type calcium ATPase functions as a core regulator of Beauveria bassiana growth, conidiation and responses to multiple stressful stimuli through cross-talk with signalling networks. Environ Microbiol 15, 967-79. doi:10.1111/1462-2920.12044

Wang, J.-J., Qiu, L., Cai, Q., Ying, S.-H., Feng, M.-G., 2015. Transcriptional control of fungal cell cycle and cellular events by $F k h 2$, a forkhead transcription factor in an insect pathogen. Sci Rep 5, 10108. doi:10.1038/ srep 10108

Wang, J.-J., Qiu, L., Cai, Q., Ying, S.-H., Feng, M.-G., 2014. Three $\alpha-1,2$-mannosyltransferases contribute differentially to conidiation, cell wall integrity, multistress tolerance and virulence of Beauveria bassiana. Fungal Genet Biol 70, 1-10. doi:10.1016/j.fgb.2014.06.010

Wang, X.-X., He, P.-H., Feng, M.-G., Ying, S.-H., 2014. BbSNF1 contributes to cell differentiation, extracellular acidification, and virulence in Beauveria bassiana, a filamentous entomopathogenic fungus. Appl Microbiol Biotechnol 98, 8657-8673. doi:10.1007/s00253-0145907-0

Wang, X.-X., Ji, X.-P., Li, J.-X., Keyhani, N.O., Feng, M.-G., Ying, S.-H., 2013. A putative $\alpha$-glucoside transporter gene BbAGT1 contributes to carbohydrate utilization, growth, conidiation and virulence of filamentous entomopathogenic fungus Beauveria bassiana. Res Microbiol 164, 480-9. doi:10.1016/j. resmic.2013.02.008

Wang, Z.-L., Lu, J., Feng, M.-G., 2012. Primary roles of two dehydrogenases in the mannitol metabolism and multi-stress tolerance of entomopathogenic fungus Beauveria bassiana. Environ Microbiol 14, 2139-50. doi:10.1111/j.1462-2920.2011.02654.x

Wang, Z.L., Zhang, L. Bin, Ying, S.H., Feng, M.G., 2013. Catalases play differentiated roles in the adaptation of a fungal entomopathogen to environmental stresses. Environ Microbiol 15, 409-418. doi:10.1111/j.14622920.2012.02848.x

WHO, 2014. Malaria. World Health Organization, Geneva.

Wu, J., Ridgway, H.J., Carpenter, M.A., Glare, T.R., 2008. Identification of novel genes associated with conidiation in Beauveria bassiana with suppression subtractive hybridization. Mycologia 100, 20-30. doi:10.3852/ mycologia.100.1.20

Xia, Y., Clarkson, J.M., Charnley, a. K., 2002. Trehalosehydrolysing enzymes of Metarhizium anisopliae and their role in pathogenesis of the tobacco hornworm, Manduca sexta. J Invertebr Pathol 80, 139-147. doi:10.1016/ S0022-2011(02)00105-2

Xiao, G., Ying, S.-H., Zheng, P., Wang, Z.-L., Zhang, S., Xie, X.-Q., Shang, Y., St Leger, R.J., Zhao, G.-P., Wang, C., Feng, M.-G., 2012. Genomic perspectives on the evolution of fungal entomopathogenicity in Beauveria bassiana. Sci Rep 2, 483. doi:10.1038/srep00483

Xie, X.-Q., Guan, Y., Ying, S.-H., Feng, M.-G., 2013. Differentiated functions of Ras 1 and Ras 2 proteins in regulating the germination, growth, conidiation, multistress tolerance and virulence of Beauveria bassiana. Environ Microbiol 15, 447-62. doi:10.1111/j.14622920.2012.02871.x

$\mathrm{Xu}, \quad$ Y., Orozco, R., Wijeratne, E.M.K., EspinosaArtiles, P., Gunatilaka, A.A.L., Stock, S.P., Molnár, I., 2009. Biosynthesis of the cyclooligomer depsipeptide bassianolide, an insecticidal virulence factor of Beauveria bassiana. Fungal Genet Biol 46, 353-364. doi:10.1016/j. fgb.2009.03.001

$\mathrm{Xu}$, Y., Orozco, R., Wijeratne, E.M.K., Gunatilaka, A.A.L., Stock, S.P., Molnár, I., 2008. Biosynthesis of the Cyclooligomer Depsipeptide Beauvericin, a Virulence Factor of the Entomopathogenic Fungus Beauveria bassiana. Chem Biol 15, 898-907. doi:10.1016/j. chembiol.2008.07.011

Yassine, H., Kamareddine, L., Osta, M.A., 2012. The mosquito melanization response is implicated in defense against the entomopathogenic fungus Beauveria bassiana. PLoS Pathog 8, e1003029. doi:10.1371/journal. 
ppat. 1003029

Ying, S.-H., Feng, M.-G., Keyhani, N.O., 2013. A carbon responsive G-protein coupled receptor modulates broad developmental and genetic networks in the entomopathogenic fungus, Beauveria bassiana. Environ Microbiol 15, 2902-2921. doi:10.1111/14622920.12169

Zaim, M., Guillet, P., 2002. Alternative insecticides: An urgent need. Trends Parasitol 18, 161-163. doi:10.1016/ S1471-4922(01)02220-6

Zhang, L.-B., Tang, L., Ying, S.-H., Feng, M.-G., 2015. Subcellular localization of six thioredoxins and their antioxidant activity and contributions to biological control potential in Beauveria bassiana. Fungal Genet Biol 76, 1-9. doi:10.1016/j.fgb.2015.01.008

Zhang, S., Widemann, E., Bernard, G., Lesot, A., Pinot, F., Pedrini, N., Keyhani, N.O., 2012. CYP52X1, representing new cytochrome P450 subfamily, displays fatty acid hydroxylase activity and contributes to virulence and growth on insect cuticular substrates in entomopathogenic fungus Beauveria bassiana. J Biol Chem 287, 13477-86. doi:10.1074/jbc.M111.338947

Zhang, S., Xia, Y., Keyhani, N.O., 2011a. Contribution of the gasl gene of the entomopathogenic fungus Beauveria bassiana, encoding a putative glycosylphosphatidylinositol-anchored beta-1,3glucanosyltransferase, to conidial thermotolerance and virulence. Appl Environ Microbiol 77, 2676-84. doi:10.1128/AEM.02747-10

Zhang, S., Xia, Y.X., Kim, B., Keyhani, N.O., 2011 b. Two hydrophobins are involved in fungal spore coat rodlet layer assembly and each play distinct roles in surface interactions, development and pathogenesis in the entomopathogenic fungus, Beauveria bassiana. Mol Microbiol 80, 811-826. doi:10.1111/j.13652958.2011.07613.x

Zhang, Y., Zhang, J., Jiang, X., Wang, G., Luo, Z., Fan, Y., Wu, Z., Pei, Y., 2010. Requirement of a mitogenactivated protein kinase for appressorium formation and penetration of insect cuticle by the entomopathogenic fungus Beauveria bassiana. Appl Environ Microbiol 76, 2262-2270. doi:10.1128/AEM.02246-09

Zhang, Y., Zhao, J., Fang, W., Zhang, J., Luo, Z., Zhang, M., Fan, Y., Pei, Y., 2009. Mitogen-activated protein kinase hogl in the entomopathogenic fungus Beauveria bassiana regulates environmental stress responses and virulence to insects. Appl Environ Microbiol 75, 37873795. doi:10.1128/AEM.01913-08

Zhou, Y.-H., Hou, L., Zhang, Y.-J., Fan, Y.-H., Luo, Z.-B., Jin, D., Zhou, Q.-S., Li, Y.-J., Wang, Y., Pei, Y., 2014. Expression and promoter characterization of $B b P a c C$, a $\mathrm{pH}$ response transcription factor gene of the entomopathogenic fungus Beauveria bassiana.
Microbiology 160, 353-61. doi:10.1099/mic.0.0711590 



\section{Chapter 3}

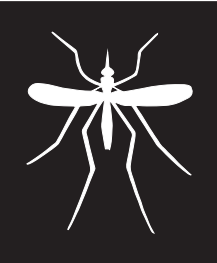

\section{Natural variation in virulence of the entomopathogenic fungus Beauveria bassiana against malaria mosquitoes}

Claudio A. Valero-Jiménez ${ }^{1,2}$, Alfons J.M. Debets ${ }^{1}$, Jan A.L. van Kan ${ }^{3}$, Sijmen E. Schoustra ${ }^{1}$, Willem Takken ${ }^{2}$, Bas J. Zwaan ${ }^{1}$ and Constantianus J.M. Koenraadt ${ }^{2}$

${ }^{1}$ Laboratory of Genetics, Wageningen University, P.O. Box 16, 6700 AA Wageningen, The Netherlands ${ }^{2}$ Laboratory of Entomology, Wageningen University, P.O. Box 16, 6700 AA Wageningen, The Netherlands

${ }^{3}$ Laboratory of Phytopathology, Wageningen University, P.O. Box 16, 6700 AA Wageningen, The Netherlands 


\title{
Chapter 3
}

\begin{abstract}
Insecticide resistance is greatly hampering current efforts to control malaria and therefore alternative methods are needed. Entomopathogenic fungi have been proposed as an alternative with a special focus on the cosmopolitan species Beauveria bassiana. However, few studies have analysed the effects of natural variation within fungal isolates on mosquito survival, and the implications and possible exploitation for malaria control. Laboratory bioassays were performed on adult female mosquitoes (Anopheles coluzzii) with spores from 29 isolates of B. bassiana, originating from different parts of the world. In addition, phenotypic characteristics of the fungal isolates such as sporulation, spore size and growth rate were studied to explore their relationship with virulence. All tested isolates of $B$. bassiana killed An. coluzzii mosquitoes, and the rate at which this happened differed significantly among the isolates. The risk of mosquitoes dying was around ten times higher when they were exposed to the most virulent as compared to the least virulent isolate. There was significant variation among isolates in spore size, growth rate and sporulation, but none of these morphological characteristics were correlated, and thus predictive, for the ability of the fungal isolate to kill malaria mosquitoes. This study shows that there is a wide natural variation in virulence of isolates of $B$. bassiana, and that selecting an appropriate fungal isolate is highly relevant in killing and thus controlling malaria mosquitoes, particularly if used as part of an integrated vector management strategy. Also, the wide variation observed in virulence offers the opportunity to better understand the molecular and genetic mechanisms that drive this variation and thus to address the potential development of resistance against entomopathogenic fungi.
\end{abstract}

\section{Keywords}

Entomopathogenic fungi; Beauveria bassiana; Vector control; Virulence 


\section{Natural variation}

\section{Introduction}

Although globally malaria mortality rates have fallen by $45 \%$ between 2000 and 2012, malaria is still killing an estimated 627,000 people each year (WHO, 2014). An effective way to alleviate the burden of malaria is to control its vector (anopheline mosquitoes) using insecticides. This can be achieved either through the use of insecticide-treated bed nets (ITNs) or through indoor residual spraying of insecticides (IRS). However, because of rapidly expanding insecticide resistance, there is a need to find alternatives to control the mosquitoes (WHO, 2014). Entomopathogenic fungi have been proposed as novel biological control agent to kill malaria mosquitoes (Kanzok and Jacobs-Lorena, 2006; Knols et al., 2010; Thomas and Read, 2007). Such fungi have already been used on a wide scale to control beetles (Castrillo et al., 2010), locusts (Lomer and Bateman, 2001) and other pest insects in agriculture.

Spores of hypocrealean entomopathogenic fungi are able to infect insects, including mosquitoes, via attachment to the insect's epicuticle (Clarkson and Charnley, 1996). Spores will penetrate the insect's cuticle by forming a germ tube and appressorium. The latter structure uses mechanical pressure and produces cuticle-degrading enzymes for further penetration (Pedrini et al., 2007; St Leger et al., 1998). Once the fungal structures reach the haemocoel, they are able to use the insect nutrients and grow. If the fungi are able to overcome the host immune defences, the host will die and saprophytic growth starts subsequently from the dead host. Finally, sporulation of the fungus takes place a few days later, depending on environmental conditions (Boomsma et al., 2014).

More specifically, the hypocrealean entomopathogenic fungi Beauveria bassiana and Metarhizium anisopliae can reduce the lifespan of mosquitoes under laboratory and field conditions (Mnyone et al., 2012), and they are also equally effective in killing insecticide-resistant mosquitoes (Farenhorst et al., 2009; Howard et al., 2011; Howard et al., 2010a). Besides the lethal effects, fungal infections reduce rodent Plasmodium sporozoite levels (Blanford et al., 2005), female fecundity and feeding propensity (Blanford et al., 2011; Scholte et al., 2006). Most likely, the latter is the result of entomopathogenic fungi that reduce the host-seeking behaviour of mosquitoes by lowering the response of olfactory receptor neurons exposed to odour cues, as demonstrated for Anopheles stephensi (George et al., 2011).

Entomopathogenic fungi kill mosquitoes relatively slowly compared to insecticides (1-2 weeks vs 1-2 days). This is potentially beneficial for controlling malaria, because the Plasmodium development time in the mosquito is about ten to 14 days (Killeen et al., 2000). Because of the delayed mortality, fungal pathogens specifically kill those mosquitoes that are old enough to transmit the parasite. Therefore, the selective pressure for survival in mosquitoes is reduced, and 


\section{Chapter 3}

thus, the probability of developing resistance against fungal infections is much lower (Read et al., 2009).

Beauveria bassiana is a cosmopolitan fungus from which more than thousand isolates have been collected from different locations and different points in time worldwide (Rehner et al., 2011). Phylogenetic analysis has revealed at least 18 different clades (S A Rehner, pers comm). However, to date it is not clear how much these fungal isolates vary in their ability to kill mosquitoes. The majority of mosquito control studies has focused on one isolate, namely IMI 391510 (Blanford et al., 2012, 2011; Farenhorst et al., 2009; Howard et al., 2010b) (except Blanford et al., 2005). It is important to study the natural variation of virulence against mosquitoes because this has an impact on the choices for selecting the optimal fungal agents for controlling malaria vectors and other mosquitoes and their diseases. The natural occurring variation in virulence can also be used to uncover the mechanisms that underpin it, which will allow the estimation of the potential for resistance development in the vector. For example, the whole genome of isolates with contrasting variation in virulence could be sequenced, and pairwise polymorphisms, deletions and Single Nucleotide Polymorphisms (SNPs) in structural and regulatory parts of genes could be analysed. Furthermore, transcriptome analysis of contrasting isolates could be performed, and linking the DNA-sequence variation to variation in gene expression could provide unique data on the fungal genes involved in insect infection and the molecular genetic mechanisms which influence virulence. This, in turn, will increase the potential for the development of entomopathogenic fungi as a biological control method against mosquitoes.

On the other hand, previous studies have shown that morphological and physiological characteristics of fungi are related to their virulence, such as hyphal growth rate, conidial viability, conidia production, conidia size, enzyme secretion among other factors (Liu et al., 2003; Quesada-Moraga and Vey, 2003; Talaei -Hassanloui et al., 2006; Varela and Morales, 1996; Zhang et al., 2011a). For instance, fungal isolates with rapid germination and high hyphal growth rate could be desirable because such fungi could infect the host more rapidly (Liu et al., 2003). Such characteristics could be used as criteria for isolate selection in addition to their virulence towards mosquitoes.

Therefore, in the current study, the virulence of 29 isolates of B. bassiana was evaluated on the malaria mosquito Anopheles coluzzii (formerly: Anopheles gambiae sensu stricto). Phenotypic characteristics of the fungal isolates such as sporulation, spore size and growth rate were measured and their correlation with virulence analysed. 


\section{Natural variation}

Table 1. Details of the tested Beauveria bassiana isolates against Anopheles coluzzii, including information from which host insect it was isolated, geographic origins and morphological characteristics.

\begin{tabular}{|c|c|c|c|c|c|}
\hline Isolate & Host insect & Geographic origins & $\begin{array}{c}\text { Size }(\mu \mathrm{M}) \\
( \pm \text { SEM })\end{array}$ & $\begin{array}{l}\text { Growth }(\mathrm{mm} / \\
\text { day })( \pm \text { SEM })\end{array}$ & $\begin{array}{c}\text { Sporulation }\left(\times 10^{6}\right. \\
\left.\text { conidia/ } / \mathrm{cm}^{2}\right)( \pm \text { SEM })\end{array}$ \\
\hline ARSEF 220 & & $\begin{array}{l}\text { Commonwealth of } \\
\text { Independent States }\end{array}$ & $2.57( \pm 0.02)$ & $2.22( \pm 0.01)$ & $3.42( \pm 1.79)$ \\
\hline ARSEF 502 & $\begin{array}{l}\text { Lepidoptera; Pyralidae; } \\
\text { Ostrinia nubilalis }\end{array}$ & China & $2.75( \pm 0.03)$ & $2.38( \pm 0.01)$ & $9.75( \pm 0.32)$ \\
\hline ARSEF 714 & $\begin{array}{l}\text { Homoptera; Delphacidae; } \\
\text { Nilaparvata lugens }\end{array}$ & China: Wuhan, Hupei & $2.34( \pm 0.00)$ & $2.54( \pm 0.07)$ & $14.69( \pm 2.00)$ \\
\hline ARSEF 721 & $\begin{array}{c}\text { Coleoptera; Chrysomelidae; } \\
\text { Diabrotica sp. }\end{array}$ & $\begin{array}{l}\text { Colombia: Cali, Valle del } \\
\text { Cauca }\end{array}$ & $2.45( \pm 0.03)$ & $2.14( \pm 0.02)$ & $4.75( \pm 1.63)$ \\
\hline ARSEF 1149 & $\begin{array}{l}\text { Lepidoptera; Noctuidae; } \\
\text { Helicoverpa armigera }\end{array}$ & Spain: Córdoba & $2.47( \pm 0.02)$ & $2.36( \pm 0.05)$ & $16.52( \pm 1.07)$ \\
\hline ARSEF 1514 & $\begin{array}{l}\text { Diptera; Muscidae; } \\
\text { Musca autumnalis }\end{array}$ & $\begin{array}{l}\text { France: Le Trait, } \\
\text { Seine-Maritime }\end{array}$ & $2.52( \pm 0.03)$ & $1.85( \pm 0.03)$ & $1.51( \pm 0.36)$ \\
\hline ARSEF 1520 & $\begin{array}{l}\text { Hemiptera; Miridae; } \\
\text { Lygus sp. }\end{array}$ & $\begin{array}{l}\text { France: Prades, } \\
\text { Pyrénées-Orientales }\end{array}$ & $2.45( \pm 0.01)$ & $2.41( \pm 0.02)$ & $6.83( \pm 0.36)$ \\
\hline ARSEF 1816 & $\begin{array}{l}\text { Coleoptera; Curculionidae; } \\
\text { Sitona discoideus }\end{array}$ & Morocco: Hauteban & $2.49( \pm 0.02)$ & $2.45( \pm 0.01)$ & $31.72( \pm 9.84)$ \\
\hline ARSEF 2075 & $\begin{array}{l}\text { Coleoptera; Chrysomelidae; } \\
\text { Ceratoma arcuata }\end{array}$ & $\begin{array}{l}\text { Brazil: Ribeira do Pombal, } \\
\text { Bahia }\end{array}$ & $2.55( \pm 0.01)$ & $2.45( \pm 0.03)$ & $9.37( \pm 2.12)$ \\
\hline ARSEF 2427 & $\begin{array}{l}\text { Homoptera; Delphacidae; } \\
\text { Nilaparvata lugens }\end{array}$ & $\begin{array}{c}\text { Indonesia: Cikampek, Java } \\
\text { Barat, Java }\end{array}$ & $2.40( \pm 0.01)$ & $2.60( \pm 0.02)$ & $24.64( \pm 4.28)$ \\
\hline ARSEF 2571 & $\begin{array}{l}\text { Lepidoptera; Lymantriidae; } \\
\text { Lymantria dispar }\end{array}$ & USA: Delaware & $2.39( \pm 0.02)$ & $2.23( \pm 0.08)$ & $13.46( \pm 1.93)$ \\
\hline ARSEF 2597 & $\begin{array}{c}\text { Lepidoptera; Hyblaeidae; } \\
\text { Hyblaea puer }\end{array}$ & India & $2.37( \pm 0.01)$ & $2.26( \pm 0.01)$ & $3.02( \pm 0.31)$ \\
\hline ARSEF 2861 & $\begin{array}{l}\text { Homoptera; Aphididae; } \\
\text { Diuraphis noxia }\end{array}$ & USA: Parma, Idaho & $2.38( \pm 0.01)$ & $2.19( \pm 0.05)$ & $4.16( \pm 1.09)$ \\
\hline ARSEF 4135 & $\begin{array}{l}\text { Coleoptera; Scarabaeidae; } \\
\text { Adoryphorus coulonii }\end{array}$ & Australia: Newtown, Tasmania & $2.47( \pm 0.01)$ & $2.30( \pm 0.05)$ & $1.97( \pm 0.62)$ \\
\hline ARSEF 4305 & Soil & $\begin{array}{c}\text { Australia: Epping Forest, } \\
\text { Tasmania }\end{array}$ & $2.43( \pm 0.02)$ & $2.46( \pm 0.09)$ & $14.74( \pm 1.78)$ \\
\hline ARSEF 4396 & Soil & Japan: Sapporo, Hokkaido & $2.37( \pm 0.02)$ & $2.71( \pm 0.02)$ & $23.11( \pm 0.54)$ \\
\hline ARSEF 4672 & $\begin{array}{l}\text { Lepidoptera; Hepialidae; } \\
\text { Oncopera intricata }\end{array}$ & Australia: Plenty, Tasmania & $2.23( \pm 0.01)$ & $2.08( \pm 0.02)$ & $1.08( \pm 0.08)$ \\
\hline ARSEF 5078 & $\begin{array}{l}\text { Lepidoptera; Pyralidae; } \\
\text { Galleria mellonella }\end{array}$ & USA: Grayland, Washington & $2.41( \pm 0.03)$ & $2.34( \pm 0.07)$ & $2.83( \pm 0.44)$ \\
\hline ARSEF 5641 & $\begin{array}{l}\text { Orthoptera; Acrididae; } \\
\text { Schistocerca gregaria }\end{array}$ & Ethiopia: Eritrea, Shelsela & $2.20( \pm 0.01)$ & $2.27( \pm 0.03)$ & $3.03( \pm 0.96)$ \\
\hline ARSEF 5642 & $\begin{array}{l}\text { Orthoptera; Acrididae; } \\
\text { Schistocerca gregaria }\end{array}$ & Ethiopia: Eritrea, Shelsela & $2.20( \pm 0.02)$ & $2.16( \pm 0.03)$ & $5.08( \pm 0.68)$ \\
\hline ARSEF 5769 & $\begin{array}{l}\text { Homoptera; Adelgidae; } \\
\text { Adelges tsugae }\end{array}$ & USA: Lovingston, Virginia & $2.23( \pm 0.01)$ & $1.79( \pm 0.05)$ & $24.82( \pm 5.89)$ \\
\hline ARSEF 6686 & Coleoptera; Scarabaeidae & $\begin{array}{l}\text { Ethiopia: Tikur Inchini, } \\
\text { Western Shoa }\end{array}$ & $2.46( \pm 0.01)$ & $2.04( \pm 0.03)$ & $4.83( \pm 1.26)$ \\
\hline ARSEF 6907 & $\begin{array}{l}\text { Isoptera; Rhinotermitidae; } \\
\text { Coptotermes formosanus }\end{array}$ & USA: Lake Charles, Louisiana & $1.95( \pm 0.01)$ & $2.45( \pm 0.05)$ & $10.29( \pm 1.51)$ \\
\hline ARSEF 8028 & $\begin{array}{l}\text { Hemiptera: Anthocoridae, } \\
\text { Anthocoris nemorum }\end{array}$ & $\begin{array}{l}\text { Denmark: Bakkegården, } \\
\text { Copenhagen, Tåstrup, Zealand }\end{array}$ & $2.26( \pm 0.01)$ & $1.77( \pm 0.03)$ & $0.74( \pm 0.08)$ \\
\hline ARSEF 8034 & $\begin{array}{l}\text { Lepidoptera; Pyralidae; } \\
\text { Galleria mellonella }\end{array}$ & $\begin{array}{l}\text { Denmark: Bakkegården, } \\
\text { Copenhagen, Tåstrup, Zealand }\end{array}$ & $2.62( \pm 0.05)$ & $1.77( \pm 0.06)$ & $0.97( \pm 0.28)$ \\
\hline ARSEF 8414 & $\begin{array}{l}\text { Coleoptera; Cerambycidae, } \\
\text { Anoplophora glabripennis }\end{array}$ & China: Wuhe, Anhui & $2.28( \pm 0.02)$ & $2.27( \pm 0.10)$ & $8.78( \pm 0.82)$ \\
\hline ARSEF 8854 & $\begin{array}{l}\text { Coleoptera; Scarabaeidae. } \\
\text { Rhopaea magnicornis }\end{array}$ & $\begin{array}{l}\text { Australia: Condong, New } \\
\text { South Wales }\end{array}$ & $2.48( \pm 0.03)$ & $2.86( \pm 0.07)$ & $13.44( \pm 3.37)$ \\
\hline ARSEF 9595 & Hymenoptera; Apoidea & China: Guizhou Province & $2.55( \pm 0.02)$ & $1.65( \pm 0.10)$ & $0.35( \pm 0.07)$ \\
\hline IMI 391510 & Coleoptera: Chrysomelidae & USA & $2.34( \pm 0.01)$ & $2.37( \pm 0.07)$ & $6.82( \pm 0.84)$ \\
\hline
\end{tabular}




\section{Chapter 3}

\section{Material and Methods}

\section{Mosquito rearing}

Anopheles coluzzii mosquitoes used in the experiments originated from Suakoko, Liberia (courtesy of the late Prof. M. Coluzzi). Larvae were reared in plastic trays of $10 \times 25 \times 8 \mathrm{~cm}$, filled with 11 of tap water at densities of approximately 0.3 larvae $/ \mathrm{cm}^{2}$. Larvae were fed on Tetramin ${ }^{\otimes}$ fish food (Tetra A G, Melle, Germany) daily, using $0.1 \mathrm{mg} /$ larva for first instar larvae and $0.3 \mathrm{mg} / \mathrm{larva}$ for the other three larval stages. Pupae were collected daily and transferred to holding cages of $30 \times$ $30 \times 30 \mathrm{~cm}$ in which adults were maintained in climate controlled rooms $\left(27 \pm 1^{\circ} \mathrm{C}, 80 \pm 10 \%\right.$ $\mathrm{RH}$ and a $12 \mathrm{hr} \mathrm{L}: \mathrm{D})$ and fed ad libitum on a $6 \%$ glucose solution.

\section{Production of Beauveria bassiana}

All isolates of $B$. bassiana used in this study were obtained from the USDA-ARS Collection of Entomopathogenic Fungal Cultures (ARSEF) (Table 1) except for isolate IMI 391510, which was kindly provided by the Bioprocess Engineering Department (Wageningen University, The Netherlands). Isolates were selected randomly from the ARSEF collection taking into account a wide geographical distribution as well as a broad array of hosts, including isolates that were found in soil. Initially the fungi were grown on Sabouraud Dextrose Agar with $1 \%$ yeast extract (SDAY) for 14 days at $27^{\circ} \mathrm{C}$ and spores were harvested with a $0.05 \%$ Tween 80 solution to make a spore suspension and kept at $-80^{\circ} \mathrm{C}$ until used.

To have sufficient spores for the experiments, all isolates were grown on solid-state fermenters, with a final yield of around 1-3 g of air-dry spores per isolate. In brief, $50 \mathrm{~g}$ hemp was weighed and $200 \mathrm{ml}$ of distilled water was added, mixed and sterilized for $30 \mathrm{~min}$ at $121^{\circ} \mathrm{C}$. Then $10 \mathrm{~g}$ of peptone and yeast extract were added and sterilized again. Once this mixture was cold, $200 \mathrm{ml}$ of a pre-autoclaved solution of glucose $(36.5 \mathrm{w} / \mathrm{w} \%)$ was added. This mixture was inoculated with $1 \mathrm{ml}$ of spore suspension $\left(1 \times 10^{8}\right.$ spores $\left./ \mathrm{ml}\right)$. It was incubated overnight at room temperature on a roller-bank. Then, the hemp was transferred into a 0.21 glass tube $(4.6 \mathrm{~cm}$ diameter; $30 \mathrm{~cm}$ height) and placed in a climate chamber set at $25^{\circ} \mathrm{C}$. After 21 days, spores were dried for four days by blowing dry air through the glass tube that contained the mixture. Spores were then harvested with sieves of various sizes $(125 \mu \mathrm{m}-1 \mathrm{~mm})$, and stored in $50 \mathrm{ml}$ tubes. The spores were kept at $4^{\circ} \mathrm{C}$ in the dark prior to use.

\section{Bioassays}

The virulence of 29 isolates of $B$. bassiana was checked by doing seven bioassays. Between three and six isolates were simultaneously compared in each bioassay. The seventh bioassay included four randomly chosen isolates to check for temporal experimental variation. In every bioassay, 


\section{Natural variation}

fungal spores of each isolate were suspended in Shellsol $\mathrm{T}^{\oplus}$ oil (Shell, The Netherlands) and standardized to $1 \times 10^{9}$ spores/ml by adjusting spore concentration after counting the suspension with a Bürker-Türk counting chamber. Spore viability was checked on SDAY plates after 18-20 hrs at $27^{\circ} \mathrm{C}$, and spores with a detectable germ tube were considered viable. Of the standardized spore solution, $0.9 \mathrm{ml}$ was applied to an A4-sized proofing paper one day before exposure. Coated papers were left to dry in a fume hood (Farenhorst and Knols, 2010). These papers were then placed in PVC tubes $(15 \mathrm{~cm}$ height and $8 \mathrm{~cm}$ diameter) and sealed with cling film on both ends. For each isolate tested, three PVC tubes (replicates) were prepared in this way. As a control, papers were coated with Shellsol $\mathrm{T}^{\bullet}$ oil only. Thirty An. coluzzii female mosquitoes (3-5 days old) were transferred with an aspirator to each PVC tube with the coated papers and exposed for three hours. After this, mosquitoes were transferred to plastic buckets $(25 \mathrm{~cm}$ height and $20 \mathrm{~cm}$ diameter), which were sealed with a nylon sock. All buckets were kept in a climate controlled chamber $\left(27 \pm 1^{\circ} \mathrm{C}, 80 \pm 10 \% \mathrm{RH}, 12 \mathrm{~h} \mathrm{~L}: \mathrm{D}\right)$, and daily mortality was checked for 14 days. Mosquitoes were fed ad libitum with a $6 \%$ glucose solution on a cotton plug. Fungal infection of dead mosquitoes was checked by dipping them for $5 \mathrm{sec}$ in $70 \%$ ethanol, incubating them on moist filter paper in sealed petri dishes at $25^{\circ} \mathrm{C}$ for five to seven days, and inspecting them for visible fungal growth.

\section{Phenotypic characterization of fungal isolates}

To determine spore size, isolates were grown on SDAY petri dishes for 14 days and spores were harvested with a $0.05 \%$ Tween 80 solution to make a spore suspension. A 1:50 dilution was made and size was measured for four replicates per fungal isolate with a Coulter Counter Z2 (Beckman Coulter). Linear growth rate was measured using 'race tubes' (Davis and Serres, 1970). The tubes were filled with $23 \mathrm{ml}$ of SDAY medium. Three tubes were inoculated for each isolate and growth was measured every week for three months. To determine sporulation, spores were harvested from a 14-day-old culture on SDAY with $0.05 \%$ Tween 80 solution and counted on a haemocytometer chamber using an optical microscope (400X). Two areas of $1 \mathrm{~mm}^{2}$ were counted for each plate, and three plates in total were counted for each isolate.

\section{Statistical analysis}

Mosquito survival was analysed using Kaplan-Meier survival analysis in SPSS (v.19) with significant differences between different isolates estimated using a Log Rank Test. Differences between the control and the infected mosquitoes were examined using a Cox Regression analysis in SPSS. Hazard ratios (HR; the daily chance of death) in comparison with the isolate IMI391510 were calculated. One-way ANOVA was conducted in R (2.12.2) to detect differences in conidia size, sporulation and linear growth rate, with the sporulation data being Log transformed before 


\section{Chapter 3}
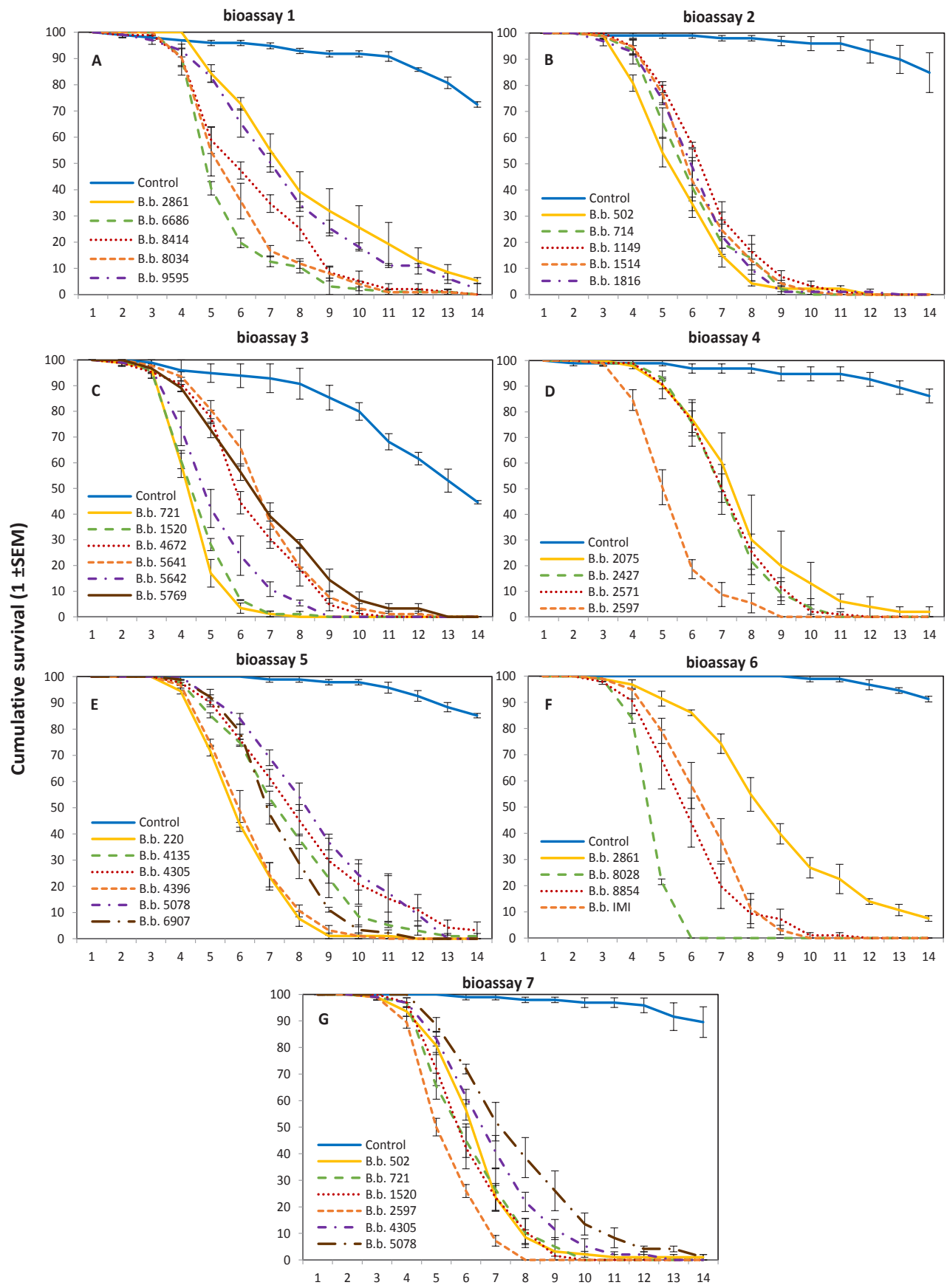

Day after exposure 
Figure 1. Cumulative daily proportional survival of Anopheles coluzzii exposed to spores of different Beauveria bassiana isolates. Control (dark blue) were exposed to only Shellsol $\mathrm{T}^{\oplus}$ oil. Exposures were carried out in seven bioassays (A-G) and 29 isolates were tested in total. Data show means \pm SEM from three replicates of 30 female mosquitoes.

analysis. A General Lineal Model was used to analyse the correlation between phenotypic characteristics of isolates and their virulence on mosquitoes using $\mathrm{R}$ (2.12.2). To further check for any correlation among the phenotypic characteristics themselves, a Pearson's Correlation analysis was done. Mosquito survival exposed to the same fungal isolates at different time points were analysed using Kaplan-Meier survival analysis in SPSS (v.19) and significant differences were estimated using a Log Rank Test.

\section{Results}

\section{Bioassays on Anopheles coluzzii}

All B. bassiana isolates tested were pathogenic to An. coluzzii with mortalities of at least $92.5 \%$ by day 14 (Figure 1). Survival curves for all mosquitoes infected with the fungus were significantly different from the respective controls for each of the seven bioassays (Figure 1). Furthermore, $74-100 \%$ of the dead mosquitoes that were exposed to fungus-coated paper showed evidence of fungal infection in the form of sporulation after five to seven days. The viability of fungal conidia of each fungal isolate was always higher than $70 \%$ at the start of the experiment. Although all bioassays were conducted in the same manner, not all controls behaved similarly in all bioassays. For unknown reasons, control mosquitoes from bioassay 1 and 3 had a significantly higher mortality compared to the controls from the other five bioassays $(F=96.09$, d.f. $=6, p<0.001$; Figure $1 \mathrm{~A}, 1 \mathrm{C})$. Therefore, these two bioassays were not considered for further analysis.

Hazard ratios (HR) were estimated relative to the mortality pattern of the reference isolate, IMI391510. This isolate was tested in detail during earlier laboratory and field trials (Farenhorst and Knols, 2010; Mnyone et al., 2012), and thus constituted a relevant reference point. Virulence was classified in three groups: (1) isolates for which the HR was significantly higher than the HR of the reference isolate (these were termed 'highly lethal' (HL)); (2) isolates that were not significantly different compared the reference isolate (these were termed 'lethal' (L)); and, (3) fungal isolates that had significantly lower HR than the reference isolate (these were termed 'slightly lethal' (SL)). The majority of the 29 isolates was classified as lethal, although at least three were HL isolates and five were SL isolates (Figure 2). Virulence varied widely, as depicted by the extremes: isolate 8028 was on average 3.7 times more virulent and isolate 2861 was on average 2.7 times less virulent than the reference isolate IMI391510. All of these isolates (8028, IMI391510 and 2861) were tested within the same bioassay (Figure 1F) removing any possibility 


\section{Chapter 3}

of temporal bias.

Isolates 502, 2597, 4305, and 5078 were randomly selected for the seventh bioassay to assess the presence of temporal variation in virulence. The two replicates of 2597 and 5078 were not significantly different from each other between the two time points and therefore were classified in the same virulence group (HL and SL, respectively). However, isolates 502 and 4305 showed significantly different HR between the temporal replicates $\left(x^{2}=10.33\right.$, d.f. $=1, \mathrm{p}=0.001 ; x^{2}=$ 6.56, d.f. $=1, p=0.010$ ), and thus, their virulence status changed. Isolate 502 was considered $\mathrm{HL}$ in the first trial, and then $\mathrm{L}$ in the second trial. The opposite effect was observed for isolate 4305 .

\section{Phenotypic characterization of fungal isolates}

Conidia of the 29 isolates of $B$. bassiana had significant differences in size, ranging from 1.95 to $2.75 \mu \mathrm{m}(\mathrm{F}=67.33$, d.f. $=28, \mathrm{p}<0.0001$, Table 1$)$. Conidia of isolates 502 and 8034 were the largest in size, whereas conidia of isolate 6907 were the smallest. Sporulation ranged from $3.5 \times 10^{5}$ to $3.17 \times 10^{7}$ spores $/ \mathrm{cm}^{2}$, and growth rate from 1.77 to $2.71 \mathrm{~mm} /$ day, with isolates differing significantly for both traits $(\mathrm{F}=10.08$, d.f. $=28, \mathrm{p}<0.0001$, and $\mathrm{F}=11.48$, d.f. $=28$, $\mathrm{p}<0.0001$, respectively). The isolate that grew fastest was 4396 and the isolate that produced the most conidia was 1816 . Nevertheless, none of these phenotypic characteristics of $B$. bassiana that were studied were correlated to the ability to kill malaria mosquitoes (i.e. virulence): sporulation $(\mathrm{F}=1.17$, d.f. $=28, \mathrm{p}=0.791)$, growth rate $(\mathrm{F}=25.47$, d.f. $=28, \mathrm{p}=0.195)$ and spore size $(\mathrm{F}$ $=22.74$, d.f. $=28, \mathrm{p}=0.169)$. Additionally, a Pearson's Correlation analysis was performed to further check for correlation among the phenotypic characteristics themselves, but none was found, except for a positive correlation between grow rate and sporulation $(r=0.704, p<0.001)$.

\section{Discussion}

Under laboratory conditions, all 29 tested isolates of B. bassiana killed An. coluzzii mosquitoes, although the rate at which this happened differed significantly among the isolates. The daily risk of death for mosquitoes when exposed to the most virulent isolate was around ten times higher than when exposed to the least virulent isolate. This observation is a substantial addition to a previous smaller scale study which tested six isolates of B. bassiana and two isolates of Metarhizium anisopliae against An. stephensi mosquitoes (Blanford et al., 2005). In that study, of two isolates of $M$. anisopliae tested, only one was pathogenic to mosquitoes. Similarly, from six B. bassiana isolates, one was not pathogenic whereas the others had different rates of virulence.

Much progress has been made regarding the feasibility of using entomopathogenic fungi as a 
ARSEF 8028

ARSEF $25972^{\text {ND }}$

ARSEF $25971^{\text {ST }}$

ARSEF 714

ARSEF $5021^{\text {ST }}$

ARSEF 220

ARSEF 1520

ARSEF 1816

ARSEF 721

ARSEF 1514

ARSEF 4396

ARSEF $5022^{\text {ND }}$

IMI 391510

ARSEF 1149

ARSEF 2427

ARSEF 2075

ARSEF $43052^{\text {ND }}$

ARSEF 2571

ARSEF 6907

ARSEF 4135

ARSEF $43051^{\text {ST }}$

ARSEF $50782^{\text {ND }}$

ARSEF $50781^{\text {ST }}$

ARSEF 2861

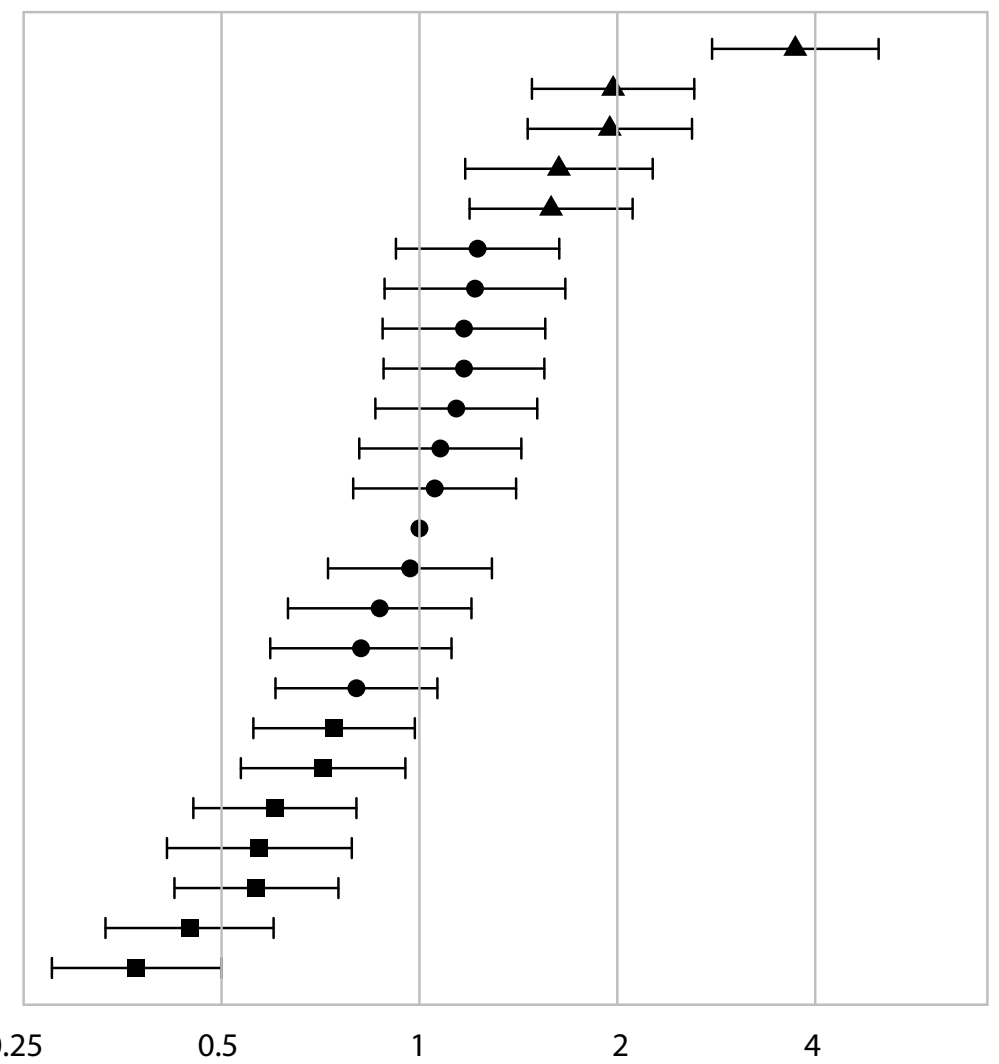

hazard ratio

Figure 2. Hazard ratios for fungal infection using isolate IMI391510 as a baseline. Dot symbols represent the isolates that were not significantly different from the reference isolate IMI391510. Triangle/square symbols show isolates that were more/less virulent than the reference isolate. Whiskers represent the $95 \%$ CIs. 1st or 2 nd indicates the result of the first or second biological replicate of an isolate.

biological control agent for malaria mosquitoes. However, researchers have thus far overlooked the potential of selecting the most suitable isolate from the existing natural variation in fungal characteristics. From the study by Blanford et al., 2005 onwards, isolate IMI391510 was selected in further experiments. This was based on practical considerations and not necessarily because it was the most virulent towards mosquitoes. For instance, this isolate was already evaluated and received regulatory approval for the control of other insects under field conditions. Follow-up studies mostly focused on isolate IMI391510 only or other isolates of $M$. anisopliae. These studies managed to standardize and improve their application under laboratory conditions (Farenhorst and Knols, 2010), test it under field conditions (Scholte et al., 2005), on insecticide-resistance 


\section{Chapter 3}

mosquitoes (Farenhorst et al., 2009; Howard et al., 2010a; Howard et al., 2011), and on different substrates such as cloth, netting, wood, clay tiles, and mud walls (Blanford et al., 2011; Mnyone et al., 2012, 2010). In the present study, it has been shown the potential of selecting the most virulent isolate since this can result in an increased effectiveness of up to ten-fold. Such selection should be dependent on the ecological situation at the location of intended use, as using a highly virulent isolate may potentially lead to an increased selection pressure that can result in resistance in mosquitoes (Thomas and Read, 2007). Additionally, genetic diversity of natural populations of mosquitoes have to be taken into consideration, since some genotypes could be more susceptible to fungal infection than others, and this could lead to an increased selective pressure as well (Mitri and Vernick, 2012). Moreover, it has been shown that there is a dose-dependent effect of spore concentration (Blanford et al., 2011; Farenhorst and Knols, 2010), so when comparing a highly lethal to a lethal isolate, the highly lethal isolate could have a similar effect using a lower concentration. Thus, the amount of spores can potentially be reduced, which would lower the application costs. Nevertheless, other factors need to be further considered since virulence is not the only variable that is important when using a fungus as biological control agent. Viability and persistence for example, can be limitations in tropical (malaria-endemic) climates.

It was hypothesized that phenotypic characteristics of $B$. bassiana potentially correlate with virulence in mosquitoes as previous studies have shown that morphological and physiological traits of B. bassiana are related to virulence in other insects (Liu et al., 2003; Varela and Morales, 1996; Zhang et al., 2011a). For example, fungal isolates with rapid germination and high hyphal growth rate may be advantageous to use as a biological control agent, because such fungi could infect the host more rapidly (Liu et al., 2003). Zhang et al., 2011a found a positive correlation between growth rate, sporulation and germination rate of $B$. bassiana with virulence of red turpentine beetle larvae. Nevertheless, in the current study no relationship could be demonstrated between morphological characteristics and the virulence of B. bassiana isolates to An. coluzzii. This is consistent with earlier work of Talaei-Hassanloui et al., 2006 who did not find any correlation between radial growth rate, spore size, germination or pigmentation and virulence on second instar larvae of Leptinotarsa decemlineata and Plutella xylostella. Ideally, one would like to be able to predict virulence of a fungus from its phenotypic characteristics without conducting bioassays that require many live insects and relatively long experimental duration before results are known. However, no such trend was observed in this study. Therefore, bioassays remain the method of choice to unravel fungal virulence, at least for this fungal-insect system and with this selection of isolates.

The results suggest that other molecular and physiological mechanisms, such as the excretion of 


\section{Natural variation}

chitinases and the ability to avoid and/or counter the insect immune response, could be related to the variation in fungal virulence. Indeed, the wide variation observed in virulence offers a unique opportunity to understand the genetic, molecular and physiological mechanisms that underpin this variation. In $B$. bassiana several proteins have been characterized as relevant for virulence. For instance, proteins of the P450 family have been identified as important for cuticle degradation (Pedrini et al., 2010; Zhang et al., 2012), and Bbslt2, a novel mitogen-activated protein (MAP) kinase, was identified to have a crucial role in regulating fungal development, growth and pathogenicity (Luo et al., 2012). As well as Bbgas1, a gene encoding a putative transferase (Glycosylphosphatidylinositol-Anchored $\beta$-1,3-Glucanosyltransferase) is involved in conidial thermotolerance and virulence (Zhang et al., 2011b). Many more genes have also been linked to virulence, which hint that virulence is a complex process regulated by several pathways

\section{Conclusions}

The results demonstrate that there is much natural variation in virulence of fungal isolates of B. bassiana that can be exploited by choosing the most suitable isolate for controlling malaria mosquitoes. This notion makes the use of entomopathogenic fungi an even more viable option, especially if used as part of an integrated vector management strategy. In addition, the natural variation observed in virulence offers the possibility to focus on the genetic mechanisms that determine this variation and would help in understanding the fungal mechanisms of B. bassiana towards malaria mosquitoes, and provide evidence that this could be an evolution-proof biological control agent. This is where the current research effort is focusing. 


\section{Chapter 3}

\section{References}

Blanford, S., Chan, B.H.K., Jenkins, N., Sim, D., Turner, R.J., Read, A.F., Thomas, M.B., 2005. Fungal pathogen reduces potential for malaria transmission. Science 308, 1638-41. doi:10.1126/science.1108423

Blanford, S., Jenkins, N.E., Christian, R., Chan, B.H.K., Nardini, L., Osae, M., Koekemoer, L., Coetzee, M., Read, A.F., Thomas, M.B., 2012. Storage and persistence of a candidate fungal biopesticide for use against adult malaria vectors. Malar J 11, 354. doi:10.1186/14752875-11-354

Blanford, S., Shi, W., Christian, R., Marden, J.H., Koekemoer, L.L., Brooke, B.D., Coetzee, M., Read, A.F., Thomas, M.B., 2011. Lethal and pre-lethal effects of a fungal biopesticide contribute to substantial and rapid control of malaria vectors. PLoS One 6, e23591. doi:10.1371/journal.pone.0023591

Boomsma, J.J., Jensen, A.B., Meyling, N.V., Eilenberg, J., 2014. Evolutionary interaction networks of insect pathogenic fungi. Annu Rev Entomol 59, 467-485. doi:10.1146/annurev-ento-011613-162054

Castrillo, L.A., Griggs, M.H., Liu, H., Bauer, L.S., Vandenberg, J.D., 2010. Assessing deposition and persistence of Beauveria bassiana GHA (Ascomycota: Hypocreales) applied for control of the emerald ash borer, Agrilus planipennis (Coleoptera: Buprestidae), in a commercial tree nursery. Biol Control 54, 61-67. doi:10.1016/j.biocontrol.2010.03.005

Clarkson, J.M., Charnley, A.K., 1996. New insights into the mechanisms of fungal pathogenesis in insects. Trends Microbiol 4, 197-203.

Davis, R., Serres, F. de, 1970. Genetic and microbiological research techniques for Neurospora crassa. Methods Enzymol 79-143.

Farenhorst, M., Knols, B.G.J., 2010. A novel method for standardized application of fungal spore coatings for mosquito exposure bioassays. Malar J 9, 27. doi:10.1186/1475-2875-9-27

Farenhorst, M., Mouatcho, J.C., Kikankie, C.K., Brooke, B.D., Hunt, R.H., Thomas, M.B., Koekemoer, L.L., Knols, B.G.J., Coetzee, M., 2009. Fungal infection counters insecticide resistance in African malaria mosquitoes. Proc Natl Acad Sci U S A 106, 1744317447. doi:10.1073/pnas.0908530106

George, J., Blanford, S., Domingue, M.J., Thomas, M.B., Read, A.F., Baker, T.C., 2011. Reduction in hostfinding behaviour in fungus-infected mosquitoes is correlated with reduction in olfactory receptor neuron responsiveness. Malar J 10, 219. doi:10.1186/14752875-10-219

Howard, A.F. V, Koenraadt, C.J.M., Farenhorst, M.,
Knols, B.G.J., Takken, W., 2010a. Pyrethroid resistance in Anopheles gambiae leads to increased susceptibility to the entomopathogenic fungi Metarhizium anisopliae and Beauveria bassiana. Malar J 9, 168. doi:10.1186/14752875-9-168

Howard, A.F. V, N'Guessan, R., Koenraadt, C.J.M., Asidi, A., Farenhorst, M., Akogbéto, M., Knols, B.G.J., Takken, W., 2011. First report of the infection of insecticide-resistant malaria vector mosquitoes with an entomopathogenic fungus under field conditions. Malar J 10, 24. doi:10.1186/1475-2875-10-24

Howard, A.F., N'guessan, R., Koenraadt, C.J., Asidi, A., Farenhorst, M., Akogbéto, M., Thomas, M.B., Knols, B.G., Takken, W., 2010b. The entomopathogenic fungus Beauveria bassiana reduces instantaneous blood feeding in wild multi-insecticide-resistant Culex quinquefasciatus mosquitoes in Benin, West Africa. Parasit Vectors 3, 87. doi:10.1186/1756-3305-3-87

Kanzok, S.M., Jacobs-Lorena, M., 2006. Entomopathogenic fungi as biological insecticides to control malaria. Trends Parasitol 22, 49-51. doi:10.1016/j.pt.2005.12.008

Killeen, G.F., McKenzie, F.E., Foy, B.D., Schieffelin, C., Billingsley, P.F., Beier, J.C., 2000. A simplified model for predicting malaria entomologic inoculation rates based on entomologic and parasitologic parameters relevant to control. Am J Trop Med Hyg 62, 535-44.

Knols, B.G.J., Bukhari, T., Farenhorst, M., 2010. Entomopathogenic fungi as the next-generation control agents against malaria mosquitoes. Future Microbiol 5, 339-341. doi:10.2217/fmb.10.11

Liu, H., Skinner, M., Brownbridge, M., Parker, B.L., 2003. Characterization of Beauveria bassiana and Metarhizium anisopliae isolates for management of tarnished plant bug, Lygus lineolaris (Hemiptera: Miridae). J Invertebr Pathol 82, 139-147. doi:10.1016/S0022-2011(03)00018-1

Lomer, C., Bateman, R., 2001. Biological control of locusts and grasshoppers. Annu Rev Entomol 46, 667-702.

Luo, X., Keyhani, N.O., Yu, X., He, Z., Luo, Z., Pei, Y., Zhang, Y., 2012. The MAP kinase Bbslt2 controls growth, conidiation, cell wall integrity, and virulence in the insect pathogenic fungus Beauveria bassiana. Fungal Genet Biol 49, 544-55. doi:10.1016/j.fgb.2012.05.002

Mitri, C., Vernick, K.D., 2012. Anopheles gambiae pathogen susceptibility: the intersection of genetics, immunity and ecology. Curr Opin Microbiol 15, 28591. doi:10.1016/j.mib.2012.04.001

Mnyone, L.L., Kirby, M.J., Lwetoijera, D.W., Mpingwa, M.W., Simfukwe, E.T., Knols, B.G.J., Takken, W., Russell, T.L., 2010. Tools for delivering entomopathogenic fungi to malaria mosquitoes: effects of delivery surfaces on fungal efficacy and persistence. Malar J 9, 246. doi:10.1186/1475-2875-9-246 


\section{Natural variation}

Mnyone, L.L., Lyimo, I.N., Lwetoijera, D.W., Mpingwa, M.W., Nchimbi, N., Hancock, P.A., Russell, T.L., Kirby, M.J., Takken, W., Koenraadt, C.J.M., 2012. Exploiting the behaviour of wild malaria vectors to achieve high infection with fungal biocontrol agents. Malar J 11, 87. doi:10.1186/1475-2875-11-87

Pedrini, N., Crespo, R., Juárez, M.P., 2007. Biochemistry of insect epicuticle degradation by entomopathogenic fungi. Comp Biochem Physiol C Toxicol Pharmacol 146, 124-137. doi:10.1016/j.cbpc.2006.08.003

Pedrini, N., Zhang, S., Juárez, M.P., Keyhani, N.O., 2010. Molecular characterization and expression analysis of a suite of cytochrome P450 enzymes implicated in insect hydrocarbon degradation in the entomopathogenic fungus Beauveria bassiana. Microbiology 156, 2549-57. doi: $10.1099 / \mathrm{mic} .0 .039735-0$

Quesada-Moraga, E., Vey, A., 2003. Intra-specific Variation in Virulence and In Vitro Production of Macromolecular Toxins Active Against Locust Among Beauveria bassiana Strains and Effects of In Vivo and In Vitro Passage on These Factors Intra-specific Var. Biocontrol Sci Technol 13, 323-340.

Read, A.F., Lynch, P. a, Thomas, M.B., 2009. How to make evolution-proof insecticides for malaria control. PLoS Biol 7, e1000058. doi:10.1371/journal.pbio. 1000058

Rehner, S. a, Minnis, A.M., Sung, G.-H., Luangsa-ard, J.J., Devotto, L., Humber, R. a, 2011. Phylogeny and systematics of the anamorphic, entomopathogenic genus Beauveria. Mycologia 103, 1055-73. doi:10.3852/10302

Scholte, E.-J., Knols, B.G.J., Takken, W., 2006. Infection of the malaria mosquito Anopheles gambiae with the entomopathogenic fungus Metarhizium anisopliae reduces blood feeding and fecundity. J Invertebr Pathol 91, 43-9. doi:10.1016/j.jip.2005.10.006

Scholte, E.-J., Ng'habi, K., Kihonda, J., Takken, W., Paaijmans, K., Abdulla, S., Killeen, G.F., Knols, B.G.J., 2005. An entomopathogenic fungus for control of adult African malaria mosquitoes. Science (80) 308, 1641-2. doi:10.1126/science.1108639

St Leger, R.J., Joshi, L., Roberts, D., 1998. Ambient pH is a major determinant in the expression of cuticledegrading enzymes and hydrophobin by Metarhizium anisopliae. Appl Environ Microbiol 64, 709-713.

Talaei -Hassanloui, R., Kharazi-Pakdel, A., Goettel, M., Mozaffari, J., 2006. Variation in virulence of Beauveria bassiana isolates and its relatedness to some morphological characteristics. Biocontrol Sci Technol 16, 525-534. doi:10.1080/09583150500532758

Thomas, M.B., Read, A.F., 2007. Can fungal biopesticides control malaria? Nat Rev Microbiol 5, 377-383. doi: $10.1038 /$ nrmicro 1638
Varela, A., Morales, E., 1996. Characterization of Some Beauveria bassiana Isolates and Their Virulence toward the Coffee Berry Borer Hypothenemus hampei. J Invertebr Pathol 152, 147-152.

WHO, 2014. Malaria. World Health Organization, Geneva.

Zhang, L.-W., Liu, Y.-J., Yao, J., Wang, B., Huang, B., Li, Z.-Z., Fan, M.-Z., Sun, J.-H., 2011a. Evaluation of Beauveria bassiana (Hyphomycetes) isolates as potential agents for control of Dendroctonus valens. Insect Sci 18, 209-216. doi:10.1111/j.1744-7917.2010.01361.x

Zhang, S., Widemann, E., Bernard, G., Lesot, A., Pinot, F., Pedrini, N., Keyhani, N.O., 2012. CYP52X1, representing new cytochrome P450 subfamily, displays fatty acid hydroxylase activity and contributes to virulence and growth on insect cuticular substrates in entomopathogenic fungus Beauveria bassiana. J Biol Chem 287, 13477-86. doi:10.1074/jbc.M111.338947

Zhang, S., Xia, Y., Keyhani, N.O., 2011b. Contribution of the gas 1 gene of the entomopathogenic fungus Beauveria bassiana, encoding a putative glycosylphosphatidylinositol-anchored beta-1,3glucanosyltransferase, to conidial thermotolerance and virulence. Appl Environ Microbiol 77, 2676-84. doi:10.1128/AEM.02747-10 



\section{Chapter 4}

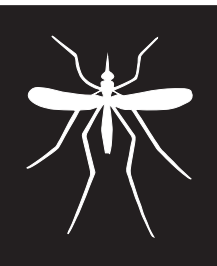

\section{Comparative genomics of Beauveria bassiana: uncovering signatures of virulence against insects}

Claudio A. Valero-Jiménez ${ }^{1,2}$, Luigi Faino ${ }^{3}$, Daphne Spring in 't Veld ${ }^{1,4}$, Sandra Smit ${ }^{4}$, Bas J. Zwaan ${ }^{1}$ and Jan A.L. van Kan ${ }^{3}$

${ }^{1}$ Laboratory of Genetics, Wageningen University, P.O. Box 16, 6700 AA Wageningen, The Netherlands ${ }^{2}$ Laboratory of Entomology, Wageningen University, P.O. Box 16, 6700 AA Wageningen, The Netherlands ${ }^{3}$ Laboratory of Phytopathology, Wageningen University, P.O. Box 16, 6700 AA Wageningen, The Netherlands

${ }^{4}$ Bioinformatics Group, Wageningen University, P.O. Box 16, 6700 AA Wageningen, The Netherlands 


\title{
Chapter 4
}

\begin{abstract}
The use of entomopathogenic fungi such as Beauveria bassiana is a promising tool for biological control of malaria mosquitoes. Indeed, infection with $B$. bassiana reduces the lifespan of mosquitoes in the laboratory and in the field. Moreover, natural isolates of $B$. bassiana show up to 10 -fold differences in virulence between the most virulent isolate and the least virulent isolate. In this study, we sequenced five isolates representing the extremes of low/high virulence, and three RNA libraries to build the transcriptome of Bb8028. Genome comparisons were done to uncover the genetic mechanisms underpinning virulence. A high-quality, near-complete genome assembly was achieved for the highly virulent isolate Bb8028 and it was compared to draft assemblies of four other isolates. Whole genome analysis showed a highly level of genetic diversity between the five isolates (2.85-16.81 SNPs/kb), which grouped into two distinct phylogenetic clusters. Mating type gene analysis revealed the presence of either the MAT 1-1-1 or the MAT 1-2-1 gene, and a putative new MAT gene was detected in the MAT 1-2 locus. Comparative genome analysis revealed that $\mathrm{Bb} 8028$ contains $0.71 \mathrm{Mb}$ (2.03\% of total genome) of unique sequences. Among the 390 unique genes of Bb8028 are a putative Non-ribosomal Peptide Synthetase (NRPS) secondary metabolite gene cluster, and several genes with homology to bacterial toxin proteins. Overall, a survey of candidate virulence genes for $B$. bassiana is presented. The genome sequences of five isolates of $B$. bassiana provides a better understanding of the natural variation in virulence, and will offer a major resource for future research about this important biological control agent.
\end{abstract}

\section{Keywords}

Beauveria bassiana; Comparative genomics; Virulence; Genome sequencing 


\section{Comparative genomics}

\section{Introduction}

Beauveria bassiana is a cosmopolitan entomopathogenic fungus (EPF) with a broad host range covering most insect orders (e.g. Coleoptera, Diptera, Hemiptera, Lepidoptera) and also arthropods (subclass Acari) (de Faria and Wraight, 2007; Kirkland et al., 2004). B. bassiana is currently used commercially as a biological control agent for different arthropod pests in China such as the pine caterpillar (Thaumetopoea pityocampa), corn borer (Ostrinia furnacalis), peach aphid (Myzus persicae), and pine sawyer (Bursaphelenchus xylophilus) (Li et al., 2010). B. bassiana is also being developed as a biological control tool for vectors of human diseases, such as Anopheles mosquitoes that transmit malaria (Blanford et al., 2005; Mnyone et al., 2012), Aedes aegypti mosquitoes, that are vectors of Dengue, Chikungunya and Zika virus (Darbro et al., 2012; de Paula et al., 2008), Triatoma infestans bug, the Chagas disease vector (Forlani et al., 2015) and Ixodes ricinus ticks that transmit Lyme disease (Hartelt et al., 2008).

Infection by $B$. bassiana starts when conidia adhere to the host cuticle via hydrophobic interactions and subsequently germinate. A germ tube is formed that is able to penetrate the cuticle through a combination of mechanical pressure and cuticle degrading enzymes. Once the fungal structures reach the haemocoel, the fungus switches to growth as single-celled blastospores, which are capable of evading the insect immune system, exploit the haemolymph for nutrition, and secrete toxins that eventually will kill the host. Once the host is dead, the fungus breaches the cuticle from the inside outwards, allowing the formation of conidia that upon dispersal can start new infections (Boomsma et al., 2014).

A good understanding on the life history of B. bassiana as well as the genetic mechanisms of virulence is crucial to develop as sustainable biocontrol agent. Consequently, substantial progress has been made in the genetic characterization of the mechanisms involved in the infection cycle of $B$. bassiana inside insects. Many of the recent findings regarding genes involved in virulence were possible due to the development of reliable protocols that allow genetic manipulation (Fang et al., 2004), and the availability of a draft genome sequence (Xiao et al., 2012). For instance, it has been found that B. bassiana expresses two hydrophobin genes, hyd1 and hyd2, that are believed to be involved in cell surface hydrophobicity, adhesion, virulence, and composition of the rodlet layer. The $\Delta h y d 1$ knock-out mutant showed decreased virulence and spore hydrophobicity but unchanged surface adhesion, while the $\Delta$ hyd 2 knock-out mutant showed reduced hydrophobicity and surface adhesion but unchanged virulence (Zhang et al., 2011). Similarly, a cytochrome P450 gene, Bbcyp52x1, was shown to function in enzymatic breakdown of lipids in the waxy layer. The $\triangle B b c y p 52 \times 1$ knock-out mutant showed reduced virulence on Galleria mellonella upon topical application of conidia, though not upon intra-hemocoel injection (Zhang et al., 2012). 


\section{Chapter 4}

Despite all efforts made to understand virulence in B. bassiana (for a review, see (Valero-Jiménez et al., 2016)), many of the genes that were functionally analysed thus far are involved in general biological processes (e.g. conidiation, stress response) that pleiotropically affect virulence. This indicates that on the one hand virulence is tightly connected to the biology and life history of Beauveria and, on the other hand, that this is generally to be expected from gene knock-out approaches. In order to increase the efficacy and specificity of Beauveria against arthropods, we need to identify the genes exclusively implicated in entomopathogenic virulence. Therefore, we need to employ strategies that allow de novo identification of genes and mechanisms specifically related to virulence. Our previous description of ample natural genetic variation in virulence in B. bassiana (Valero-Jiménez et al., 2014) in combination with comparative genomic approaches offers a powerful opportunity to collect comprehensive information of genetic variation and identification of variants of virulence and their evolutionary dynamics. Importantly, knock-out mutant approaches are crucial and will play an important role in verifying the action of the candidate genes that will emerge from comparative genomics of natural isolates that differ in virulence.

The aim of this study was to improve our knowledge in understanding the key factors involved in virulence of $B$. bassiana by a comparative genomics study. B. bassiana is well known for having a broad genetic, host, and virulence diversity. As a consequence, genome structure and DNA sequence variation between any two isolates could be related to many factors in the biology of this fungus (including neutral evolution/genetic drift). Therefore, we sequenced five isolates of contrasting virulence, selected from a panel of 29 B. bassiana isolates of which the natural variation in virulence was characterized (Valero-Jiménez et al., 2014). We assembled a gapless near-complete genome for the most virulent B. bassiana isolate, Bb8028, which provides the community a suitable reference genome to be used for comparison with other isolates.

\section{Materials and Methods}

\section{Fungal isolates and maintenance}

Beauveria bassiana isolates ARSEF 1520 (Bb1520), ARSEF 2597 (Bb2597), ARSEF 4305 (Bb4305), ARSEF 5078 (Bb5078), and ARSEF 8028 (Bb8028) were obtained from the USDAARS Collection of Entomopathogenic Fungal Cultures (ARSEF). These isolates were selected based on their previously characterized virulence against malaria mosquitoes (Valero-Jiménez et al., 2014), in which isolates Bb2597 and Bb8028 are considered highly virulent, Bb1520 intermediate virulence, and $\mathrm{Bb} 4305$ and $\mathrm{Bb} 5078$ are considered to be lowly virulent. Isolates were grown on Sabouraud Dextrose Agar with $1 \%$ yeast extract (SDAY) at $27^{\circ} \mathrm{C}$ before DNA extraction and sequencing. 


\section{Comparative genomics}

\section{Genome and transcriptome sequencing}

All isolates were sequenced with paired-end libraries of $180 \mathrm{bp}$ insert size, and with a read length of 2x100 bp. Isolates Bb1520, Bb2597, and Bb8028 were further sequenced with a mate-pair library of $10 \mathrm{~kb}$ or $6 \mathrm{~kb}$ (Bb8028). Isolate $\mathrm{Bb} 8028$ was selected as a reference based on the quality of the assembly, therefore additional sequencing was performed using two PacBio SMRT cells with an insert size of $20 \mathrm{~kb}$. The paired-end and mate-pair sequencing were performed using Illumina HiSeq2000 technology at BGI Company and PacBio sequencing was performed using the RS II instrument at Keygene N.V. (Wageningen, the Netherlands).

To obtain the transcriptome, RNA from Bb8028 was isolated from three different sources: (1) 5-day old mycelia grown on rice medium; (2) proto fruiting bodies (stromata like-structures) obtained from a cross between Bb8028 and Bb5078 grown for 2 months on rice media following the methods of Lee et al., (2010); and (3) freshly dead infected mosquitoes. The rice medium was composed of $60 \mathrm{gr}$ of brown rice and $10 \mathrm{gr}$ of dried silkworm pupae and $100 \mathrm{ml}$ of distilled water. RNA was sequenced using a paired-end library of 200 bp insert size using Illumina HiSeq2000 technology at BGI Company.

\section{Genome assembly and annotation}

Different assembly strategies were used based on the data generated for each isolate. For isolates Bb4305 and Bb5078, a de novo assembly was performed using Celera assembler (Myers, 2000) with default settings except k-mer size, which was set to 15. Isolate Bb1520, Bb2597 and Bb8028 were assembled using Allpaths-LG (Ribeiro et al., 2012) using default settings. Additionally, PacBio data was used to improve the assembly of Bb8028 using PBJelly (English et al., 2012) and quiver tools (Chin et al., 2013), and erroneously merged contigs were manually corrected. RNASeq libraries were assembled de novo with Oases (Schulz et al., 2012) and Trinity (Grabherr et al., 2011). Also, the RNA-Seq reads were mapped to the Bb8028 assembly using Tophat (Trapnell et al., 2009). Completeness of the genome assemblies was assessed by the Benchmarking Universal Single-Copy Orthologs (BUSCO) v1.1b1 software tool (Simão et al., 2015). RepeatMasker (Smit et al., 2013) was used to search for homologs of known repeats using the library from Repbase (Jurka et al., 2005), and previously unknown repeats were predicted de novo with RepeatScout (Price et al., 2005).

Genome annotation was performed using the MAKER (v.2.31.8) pipeline (Cantarel et al., 2008) with AUGUSTUS (Stanke et al., 2006), SNAP (Korf, 2004), and GeneMark-ES (TerHovhannisyan et al., 2008) as ab initio gene predictors. The assembled RNA-Seq reads and all fungal proteins available in the Uniprot database were used as evidence for gene prediction. 


\section{Chapter 4}

Furthermore, the predicted proteins were manually inspected and curated. The predicted proteins were functionally annotated using BLASTp (Altschul et al., 1990) against the non-redundant database of the National Center of Biotechnology Information (NCBI) and InterProScan (Jones et al., 2014). The BLASTp and InterproScan results were imported into Blast2GO (Conesa et al., 2005) and the Gene Ontology (GO) terms were predicted. Finally, secreted proteins were predicted by SignalP 4.1 (Petersen et al., 2011).

\section{Pan-genome analysis}

The pan-genomes and core genomes at the gene level were estimated using the OrthoMCL (Li, 2003) algorithm implemented in GET_HOMOLOGUES-EST (Contreras-Moreira and Vinuesa, 2013) with parameters: e-value: $1 \mathrm{e}^{-5}$, and coverage: $75 \%$. The genome of $B$. bassiana ARSEF 2860 (Bb2860) (Xiao et al., 2012) was also included in the analysis.

\section{Phylogenetic analysis}

The phylogenetic relationships were determined with the software REALPHY (Bertels et al., 2014) between all isolates of B. bassiana, including Bb2860, and C. militaris (Zheng et al., 2011) as the outgroup of the tree. All genomes were mapped using Bowtie2 (Langmead et al., 2009) on the genome of Bb8028 as a reference. After the alignment, a maximum likelihood phylogenetic tree was inferred with RAxML (Stamatakis, 2006) using the generalised time reversible (GTR) plus GAMMA nucleotide substitution model and 100 rapid bootstraps.

\section{Variant analysis}

To identify single-nucleotide polymorphisms (SNPs), Freebayes (v0.9.21), a Bayesian method based program, was used (Garrison and Marth, 2012) with a minimum alteration fraction of 0.90. All sequences were mapped with BWA (Li and Durbin, 2009) to the genome of isolate Bb8028. To minimize false positives, SNPs called at positions with low coverage $(<10 \mathrm{x})$ were not considered. SNP density and transition/transversion ratios were calculated using VCFtools (Danecek et al., 2011).

\section{Secondary metabolites analysis}

Putative gene clusters that are involved in biosynthesis of secondary metabolites were identified using antiSMASH (antibiotics and Secondary Metabolite Analysis SHell) (Weber et al., 2015).

\section{Mating type gene analysis}

The presence of mating type loci in all isolates of B. bassiana (including Bb2860) were identified by homology to characterized MAT genes of other filamentous fungi using BLASTp. The mating 


\section{Comparative genomics}

type alleles MAT1-1-1 and MAT1-2-1 were also determined by PCR with the primer pairs MAT112.F4/ MAT111.R5 and MAT2.F4/ MAT2.R5 (Meyling et al., 2009), respectively.

\section{Selection analysis}

To detect regions under positive selection, protein-coding DNA alignment based on the orthologous genes were generated with ParaAT. Ratios of non-synonymous $(\mathrm{Ka})$ to synonymous (Ks) nucleotide substitutions rates per site were estimated for each alignment using KaKs_ Calculator 2.0 (Wang et al., 2010). A sliding window of 100 bp was used with a gamma distribution (gMYN) model.

\section{Data availability}

The raw data for this study are deposited in NCBI Sequence Read Archive under BioProject number PRJNA260878. Bb8028: SRR3269141, SRR3269142, SRR3269778, SRR3269779, SRR3269780, SRR3269861, and SRR3269862. Bb1520 data: SRR3269145 and SRR3269146. Bb2597: SRR3269425 and SRR3269550. Bb4305: SRR326914. Bb5078: SRR3269144. This Whole Genome Shotgun project has been deposited at DDBJ/ENA/GenBank under the accessions: JRHA00000000, JTCW00000000, JTCX00000000, JTCY00000000, and JTCZ00000000.

\section{Results}

\section{Genome features}

We performed the sequencing of five $B$. bassiana isolates that were previously phenotyped for virulence against malaria mosquitoes. Isolate $\mathrm{Bb} 8028$, which is consistently the most virulent isolate in our collection (Valero-Jiménez et al., 2014) was selected to serve as a high quality reference genome for the dataset. This isolate was sequenced with both short and long-reads, single molecule sequencing technology, while two isolates were sequenced with short reads on both short and long insert libraries, and two isolates were sequenced with short reads only on short insert libraries. The final assembly sizes were in the range of 34.45-38.83 Mb, slightly larger than the assembly of the previously sequenced isolate of B. bassiana, Bb2860 (Xiao et al., 2012). The final assemblies resulted in 12 to 3,952 contigs (Table 1), and the five isolates were predicted to encode between 10,210 and 10,831 protein-coding genes. The genome assembly of isolate Bb8028 had only 12 contigs with an N50 contig length of $5.69 \mathrm{Mb}$, and there were no gaps present. The 12 contigs covering the genome of $\mathrm{Bb} 8028$ represent four full chromosomes (with telomeric repeats at both ends) and five chromosome ends with a single telomeric repeat. The completeness of all five genomes was assessed by checking for the representation of a set of universal single-copy orthologs for fungi (Simão et al., 2015), and this analysis indicated that 


\section{Chapter 4}

Table 1. Summary of the genomic features of the different B. bassiana isolates.

\begin{tabular}{llllll}
\hline Genome features & $\mathbf{B b 1 5 2 0}$ & $\mathbf{B b 2 5 9 7}$ & $\mathbf{B b 4 3 0 5}$ & $\mathbf{B b 5 0 7 8}$ & $\mathbf{B b 8 0 2 8}$ \\
\hline Total Assembly Size (Mb) & 36.97 & 38.83 & 34.77 & 34.45 & 35.02 \\
Sequencing libraries used & $\mathrm{PE}+\mathrm{MP}$ & $\mathrm{PE}+\mathrm{MP}$ & $\mathrm{PE}$ & $\mathrm{PE}$ & $\mathrm{PE}+\mathrm{MP}+\mathrm{PB}$ \\
Scaffold No. & 251 & 351 & 1,694 & 3,365 & - \\
Scaffold N50 (kb) & 1,560 & 970 & 58 & 31 & - \\
Contig No. & 1,218 & 1,356 & 2,252 & 3,952 & 12 \\
Contig N50 (kb) & 85 & 69 & 35 & 22 & 5,685 \\
GC content (\%) & 49.64 & 49.11 & 49.56 & 51.06 & 48.66 \\
Protein coding genes & 10,599 & 10,753 & 10,513 & 10,831 & 10,210 \\
Predicted secreted proteins & 1097 & 1063 & 1087 & 1069 & 1075 \\
\hline
\end{tabular}

the completeness of each genome was at least 96\% (Table S1), suggesting a high quality of the assemblies.

The pan-genome is here defined as the combination of all the genes that are present in the genomes of all isolates of a particular species, and it includes the core genes, dispensable genes, and isolate-specific genes (Vernikos et al., 2015). A pan-genome analysis for B. bassiana for the five isolates sequenced in this study in combination with the previously sequenced isolate $\mathrm{Bb} 2860$ (Xiao et al., 2012), indicated that the core genome of B. bassiana consists of 7,341 orthologous gene clusters (Fig. 1A). On the other hand, the pan-genome consists of 13,068 orthologous gene clusters (Fig. 1B). This shows that B. bassiana has an open pan-genome, as could be expected for a species that typically colonize multiple environments (Medini et al., 2005).

\section{SNPs and phylogenetic analysis}

The phylogenetic relationships between isolates of B. bassiana were determined using the whole genome. Two distinct clusters were formed (Fig. 2), designated 1 and 2. Cluster 1 contains three isolates (Bb2597, Bb5078, and Bb8028), while the other three isolates (Bb1520, Bb4305, and Bb2860) are grouped in cluster 2. The grouping did not reflect a geographical distribution: the two isolates originating from U.S.A. were assigned to different clusters, as well as the two isolates of European origin. All the read data that were available in this study were mapped to Bb8028 as a reference in order to detect SNPs. Within cluster 1, there were 2.85 and $4.81 \mathrm{SNPs}$ per $\mathrm{kb}$ for isolates $\mathrm{Bb} 5078$ and $\mathrm{Bb} 2597$, respectively. In contrast, the genetic variation from isolates from cluster 2 against Bb8028 was four-fold higher, with an average of 16.63 and 16.81 SNPs per kb, for $\mathrm{Bb} 1520$ and $\mathrm{Bb} 4305$, respectively.

\section{Mating type}

Analysis of orthologous relationships between the genes of all isolates of $B$. bassiana in this study 


\section{Comparative genomics}
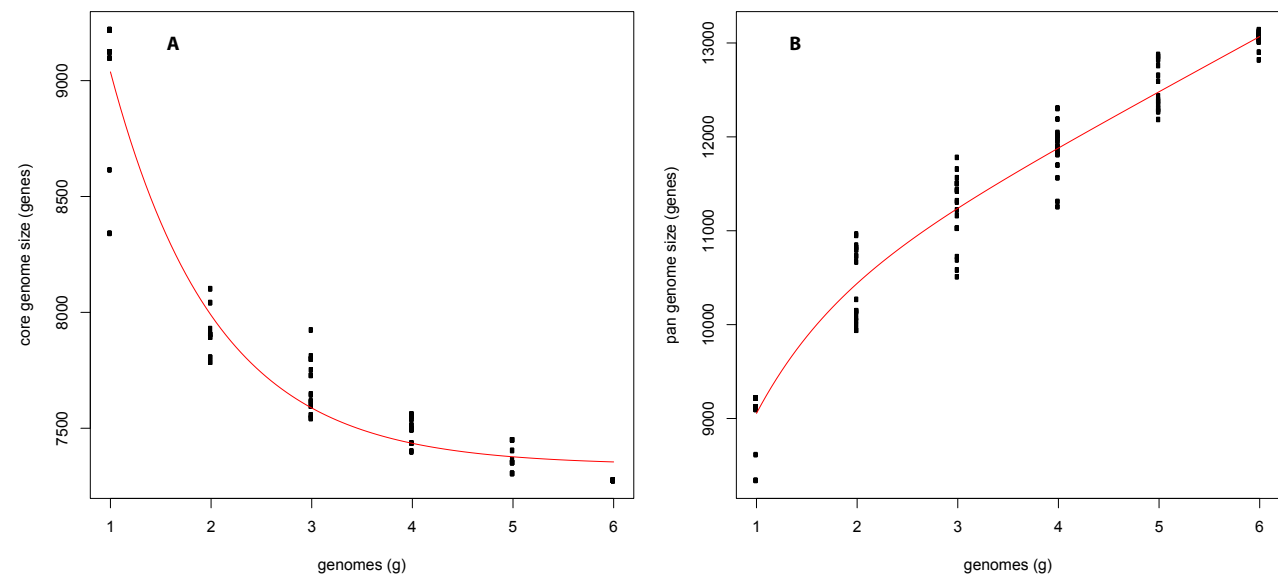

Figure 1. Pan-genome analysis of B. bassiana isolates. (A) Estimation of the B. bassiana core genome, in which the number of shared genes is plotted as a function of the number of isolates sequentially added. (B) Estimation of $B$. bassiana pan-genome size, in which the number of all genes are plotted as a function of the number of isolates sequentially added.

identified the presence of several mating type loci (Fig. 3). We identified two MAT genes, MAT 1-1-1 and MAT 1-1-2, in Bb2597 and Bb5078. Previously, the MAT 1-1-2 gene was not identified in the published genome of $\mathrm{Bb} 2860$, but based on BLAST hits, genes from Bb2597 and Bb5078 have homology to the MAT 1-1-2 gene in C. militaris (Zheng et al., 2011). Further sequence alignment of the putative MAT-1-1-2 gene from Bb2597 and Bb5078 to scaffold BBA_S00027 from $\mathrm{Bb} 2860$ confirmed that this gene is also present in Bb2860 but was not characterized by Xiao et al. (2012). The MAT 1-2-1 locus was identified by homology to the HMG-box domain in $\mathrm{Bb} 1520, \mathrm{Bb} 4305$, and Bb8028. A putative new MAT gene was found next to the MAT 1-2-1 gene, and BLASTp analysis shows that it has partial homology to MAT 1-1-1 and MAT 1-2-1. PCR confirmed the presence of either MAT 1-1-1 or MAT 1-2-1 in each isolate. The presence of each MAT locus in different $B$. bassiana isolates indicates it to be putatively heterothallic.

\section{Isolate specific genes from $\mathrm{Bb8028}$}

Isolate Bb8028 was previously characterized as the most virulent towards malaria mosquitoes (Valero-Jiménez et al., 2014). Therefore, we hypothesised that the high virulence could be due to presence of genes unique to Bb8028 that are related to fungal virulence. In order to detect regions only present in Bb8028, all reads from the other isolates were mapped to the genome assembly of Bb8028 and regions with very few or no read coverage were considered as unique to Bb8028. A total sequence length of $0.71 \mathrm{Mb}$ (2.03\% of total genome) was identified as being unique to Bb8028, and it contained 390 genes (Supplementary Data 2). Functional annotation of the unique genes of $\mathrm{Bb} 8028$ was done to evaluate their potential role in pathogenicity. Of the 


\section{Chapter 4}

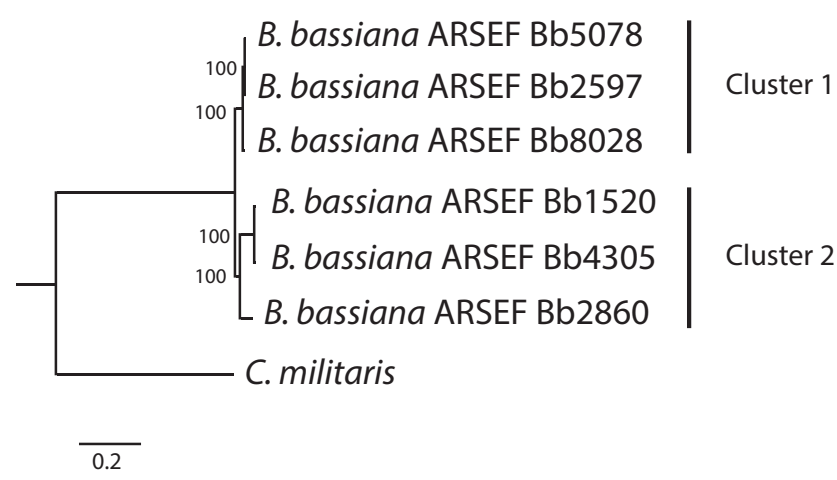

Figure 2. Phylogenetic tree based on whole genome assemblies of the different isolates of B. bassiana, with C. militaris as the outgroup to root the tree. On the right, the different clusters found are highlighted.

390 genes found to be unique to Bb8028, 108 (27.7\%) had homology to genes in the Pathogen Host Interaction Database (PHI-base), 88 (22.6\%) were hypothetical proteins, and 59 (15.1\%) had predicted signal peptides. There was a higher number of genes classified as hypothetical proteins, and with signal peptides compared to the complete gene content $(15.2 \%$ and $10.5 \%$, respectively), but similar number of genes homologous to PHI-base as in the whole gene set (29.5\%). An overrepresentation of gene ontology terms was not detected.

Secondary metabolites play a role in the fitness and virulence of fungal entomopathogens, since they can participate in counteracting host defences and in competition with other microorganisms (Molnár et al., 2010). A putative Non-ribosomal Peptide Synthetase (NRPS) secondary metabolite cluster was found in the unique gene set of Bb8028. The NRPS gene (BB8028_0008g00080) has homology (52-54\%) to several Metarhizium species based on BLASTp results, with the difference that BB8028_0008g00080 has one adenylation domain and is 1059 amino acids in length, while its Metarhizium homologs possess two adenylation domains and encode proteins almost double in size. Interestingly, the third top Blast hit was annotated as Tyrocidine synthase (KJK81377). Since tyrocidine has antibiotic activity, the peptide synthesized by the gene product of KJK81377 could putatively have antibiotic activity.

Seven unique genes of $\mathrm{Bb} 8028$ were homologous to bacterial-like toxin proteins. Six genes were homologous to a heat-labile enterotoxin (IPR001144), and one gene had homology to Deltaendotoxin CytB (IPR001615), which was originally studied in Bacillus thuringiensis as an active toxin against larvae of insects ( $\mathrm{Li}$ et al., 1996). Remarkably, putative enterotoxin genes have homology almost exclusively to Metarhizium and other entomopathogenic fungi, while the putative $\mathrm{CytB}$ endotoxin has homology to a wide range of fungi including Basidiomycetes and 


\section{Comparative genomics}

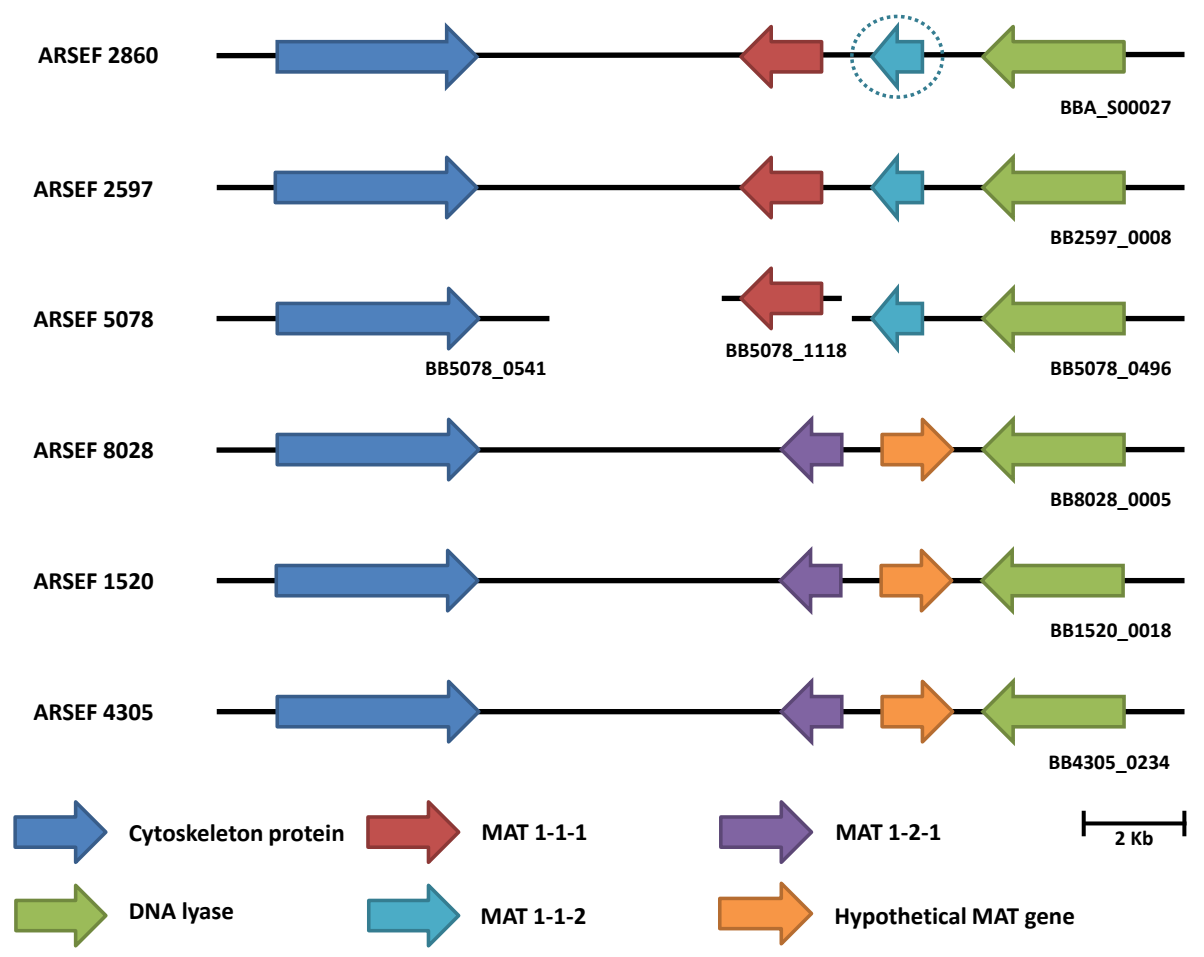

Figure3. Syntenyanalysisofthematingtypegenesand theirflankingregionin B. bassianagenomes. Genesinthesamecolourshow orthologous relationships. The dashed circle represents agene that was not previouslyidentified in thegenomeofARSEF 2860 .

other Ascomycetes not reported to display a pathogenic lifestyle.

Bb8028 contains 18 unique genes involved in transport processes, of which nine belong to the major facilitator superfamily (MFS), four to the family of ATP-binding cassette (ABC) transporters, and the remaining five are general transporters. $\mathrm{ABC}$ transporters can be involved in resistance to fungicides, as well as the secretion of antibiotics and toxins (Del Sorbo et al., 2000). Recently, five ABC transporters were characterized in B. bassiana and were found to be implicated in fungicide resistance, oxidative stress tolerance, and in virulence (Song et al., 2013). Moreover, a number of genes involved in detoxification of compounds, which could be related to interactions to insect hosts, were found uniquely in Bb8028, such as six dehydrogenases, one monooxygenase, one acetyltransferase, and one cytochrome P450 gene. 


\section{Chapter 4}

Although a detailed analysis of differential gene expression could not be done with the RNA data available due to the lack of sample replication, an exploratory examination was done to see whether unique genes of Bb8028 were highly expressed. Specifically, we looked for genes that had at least (1) double the expression (in Fragments Per Kilobase of transcript per Million mapped reads (FPKM)) in the library isolated from infected mosquitoes compared against the fruiting body or mycelia library, and (2) a value of at least one FPKM. Of the 390 genes, only 42 genes fulfilled these criteria. Notably, five of the seven genes described as a toxin were among these 42 genes, and the gene with the highest expression in infected mosquitoes is the Deltaendotoxin gene mentioned above (Supplementary Data 3).

\section{Positive selection}

Genes with a signature of positive selection may contribute to the adaptation to a changing environment and could be related to virulence. Therefore, we investigated which genes were under positive selection in $\mathrm{Bb} 8028$. We calculated the $\mathrm{Ka} / \mathrm{Ks}$ ratio by comparing the orthologs between $\mathrm{Bb} 8028$ and each of the four other isolates (Bb1520, Bb2597, Bb4305, Bb5078) and respectively detected 10, 5, 11, 10 genes of $\mathrm{Bb} 8028$ with a $\mathrm{Ka} / \mathrm{Ks}$ ratio > 1 . In total, 19 genes are considered to be under selection in Bb8028, but intriguingly no gene overlapped among the four gene sets (Supplementary Data 4). One of the genes under positive selection is annotated as an $\mathrm{ABC}$ transporter, while most of the genes found were of unknown function.

\section{Discussion}

In this study, we provide and compare the genomes of five isolates of B. bassiana that were previously characterized in a large-scale analysis of virulence against the malaria mosquito (An. coluzzii). These isolates have different levels of virulence, with Bb8028 being the most virulent isolate, followed by $\mathrm{Bb} 2597, \mathrm{Bb} 1520, \mathrm{Bb} 4305$, and $\mathrm{Bb} 5078$. Comparative genomics at the intraspecific level is an approach to determine the genetic basis of phenotypic variations detected between different isolates. The genome of $B$. bassiana was previously compared to other entomopathogenic fungi (Xiao et al., 2012), and in order to avoid a duplication of this previous work, we focused on comparison of B. bassiana isolates, with a focus on Bb8028, which is the most virulent isolate.

Genome analysis shows that the isolates have a high level of diversity, with 467,292 SNPs between Bb4305 and Bb8028, and 87,119 SNPs between Bb5078 and Bb8028. Previous studies have shown that B. bassiana is not monophyletic, however it is composed of several cryptic species that are morphologically identical (Ghikas et al., 2010; Meyling et al., 2009; Rehner et al., 2006). For instance, Ghikas et al. (2010) described seven phylogenetic clusters in B. bassiana that 


\section{Comparative genomics}

Table 2. Fruiting body formation from isolates of Beauveria bassiana with different mating types. - indicates no fruiting body formation, and $+/$ - indicates fruiting body formation without ascospores.

\begin{tabular}{lll}
\hline Isolates & Bb2597 & Bb5078 \\
\hline Bb1520 & - & - \\
Bb4305 & - & - \\
Bb8028 & - & $+/-$ \\
\hline
\end{tabular}

consisted of isolates which did not show any geographic distribution but had common climate characteristics, and studies from Meyling et al. (2009) found isolates that were distributed in five clusters in a particular hedgerow habitat. Furthermore, a comparative genomic study of seven isolates of the plant pathogen Penicillium expansum showed that the genetic diversity found was between 1.27-7.13 SNPs/kb (Julca et al., 2016), while in two isolates of Aspergillus niger it was on average 7.8 SNPs/kb (Andersen et al., 2011). In both cases, the genetic diversity observed was lower than the level found in this study (2.85-16.81 SNPs/kb). Consequently, is not surprising that based on only six isolates, we could distinguish two clusters that had different geographic and host origins, which corroborates the findings that B. bassiana possess a high genetic diversity, and warrants further studies to elucidate the phylogenetic history of the species.

B. bassiana normally reproduces asexually, and for a long time was believed to be exclusively clonal, but developmental and phylogenetic studies have linked it to the teleomorph Cordyceps bassiana (Rehner and Buckley, 2005; Sung et al., 2006). In the five isolates studied, we identified the MAT1-1 and MAT 1-2 loci based on homology to the typical fungal mating type genes, encoding the alpha domain protein and to the high mobility group box domain, respectively. We attempted to cross different isolates of $B$. bassiana in several combinations to generate fertile fruiting bodies. Based on the mating type, only six possible combinations were possible (Table 2). Unfortunately, none of the crosses generated ascospores, but the cross between Bb8028 and $\mathrm{Bb} 5078$ generated fruiting body-like structures without ascospores, that were used to generate a transcriptome library. When taking into consideration the phylogenetic clusters found, we realized that only Bb8028 and B5078 belong to the same cluster and had the lowest number of SNPs/kb, compared to the other possible combinations. If we take into consideration that B. bassiana could be polyphyletic, and reports have found a single mating type in several populations (Meyling et al., 2009), it is not surprising that B. bassiana is limited mainly to asexual reproduction. The ability to obtain sexual reproduction under lab conditions would have been useful to perform GWAS of quantitative virulence between offspring of two isolates that differ in virulence, e.g. between $\mathrm{Bb} 8028$ and $\mathrm{Bb} 5078$.

In this analysis, we identified a suite of genes, which are unique to Bb8028 of which several 


\section{Chapter 4}

may have putative virulence functions. A more detailed characterization of the unique genes showed that several could have an important role in virulence such as the NRPS cluster found, as well as seven bacterial-like toxins, and 18 transporter genes. The B. bassiana genome contains many more bacterial-like toxins than other fungi (Xiao et al., 2012). Interestingly, we found preliminary evidence that some of the bacterial-like toxins are highly expressed in fungal infection of mosquitoes, which could be crucial for the infection cycle. A recent study (Chu et al., 2016) analysed the gene expression of B. bassiana ARSEF 2860 after infection of Plutella xylostella larvae at three different time points (24h, 36h, 48h). In this study, an enrichment of genes with functions in antioxidant activity, peroxidase activity, and proteolysis was detected among the genes that are highly expressed in B. bassiana during infection of the host insect. Furthermore, KEGG enrichment analyses were performed, and the majority of pathways that are highly expressed in B. bassiana during infection of the host insect were related to energy metabolism and biosynthesis, but there was no clear indication of putative genes involved in virulence (Chu et al., 2016). Mortality of $P$. xylostella larvae infected by B. bassiana at $48 \mathrm{~h}$ was $33 \%$, compared to our infected mosquito RNA library that was sampled at 6 days post infection with a mortality of almost $100 \%$. The infection cycle of $B$. bassiana is a complex process that includes several developmental transitions that requires an intricate genetic regulation (Valero-Jiménez et al., 2016), which could explain why no overlap is found between the studies. Furthermore, $B$. bassiana is capable of infecting a wide range of hosts that have different chemical and physical compositions, which most likely requires a tailored response and host-pathogen interactions.

The availability of a near-complete genome assembly of Bb8028 provided with a manually curated gene set, adds significant value to the previously published genome of $B$. bassiana (Xiao et al., 2012). As argued by Thomma et al. (2015), a high quality assembly including proper assembly of centromeres, telomeres and other repetitive elements provides the ability to characterize structural rearrangements and a better identification of secondary metabolite gene clusters. Therefore, a high quality genome is a key step to uncover the biological complexity of an organism. The Bb8028 genome assembly represents a significant resource for future research into the biology of this entomopathogenic fungus and its relevance as a biological control agent. Understanding the key factors involved in virulence of $B$. bassiana will improve our methods to use this fungus as an effective biological control agent against vectors of human disease. 


\section{Comparative genomics}

\section{References}

Altschul, S.F., Gish, W., Pennsylvania, T., Park, U., 1990. Basic Local Alignment Search Tool. Department of Computer Science 403-410. doi:10.1016/S00222836(05)80360-2

Andersen, M.R., Salazar, M.P., Schaap, P.J., Van De Vondervoort, P.J.I., Culley, D., Thykaer, J., Frisvad, J.C., Nielsen, K.F., Albang, R., Albermann, K., Berka, R.M., Braus, G.H., Braus-Stromeyer, S.A., Corrochano, L.M., Dai, Z., Van Dijck, P.W.M., Hofmann, G., Lasure, L.L., Magnuson, J.K., Menke, H., Meijer, M., Meijer, S.L., Nielsen, J.B., Nielsen, M.L., Van Ooyen, A.J.J., Pel, H.J., Poulsen, L., Samson, R.A., Stam, H., Tsang, A., Van Den Brink, J.M., Atkins, A., Aerts, A., Shapiro, H., Pangilinan, J., Salamov, A., Lou, Y., Lindquist, E., Lucas, S., Grimwood, J., Grigoriev, I. V., Kubicek, C.P., Martinez, D., Van Peij, N.N.M.E., Roubos, J.A., Nielsen, J., Baker, S.E., 2011. Comparative genomics of citric-acid-producing Aspergillus niger ATCC 1015 versus enzyme-producing CBS 513.88. Genome Res 21, 885-897. doi:10.1101/gr.112169.110

Bertels, F., Silander, O.K., Pachkov, M., Rainey, P.B., van Nimwegen, E., 2014. Automated reconstruction of whole genome phylogenies from short sequence reads. Mol Biol Evol 31, 1077-1088. doi:10.1093/molbev/ msu088

Blanford, S., Chan, B.H.K., Jenkins, N., Sim, D., Turner, R.J., Read, A.F., Thomas, M.B., 2005. Fungal pathogen reduces potential for malaria transmission. Science 308, 1638-41. doi:10.1126/science.1108423

Boomsma, J.J., Jensen, A.B., Meyling, N. V, Eilenberg, J., 2014. Evolutionary Interaction Networks of Insect Pathogenic Fungi. Annu Rev Entomol 467-485. doi:10.1146/annurev-ento-011613-162054

Cantarel, B.L., Korf, I., Robb, S.M.C., Parra, G., Ross, E., Moore, B., Holt, C., Alvarado, A.S., Yandell, M., 2008. MAKER: An easy-to-use annotation pipeline designed for emerging model organism genomes. Genome Res 18, 188-196. doi:10.1101/gr.6743907

Chin, C.-S., Alexander, D.H., Marks, P., Klammer, A.A., Drake, J., Heiner, C., Clum, A., Copeland, A., Huddleston, J., Eichler, E.E., Turner, S.W., Korlach, J., 2013. Nonhybrid, finished microbial genome assemblies from long-read SMRT sequencing data. Nat Methods 10, 563-569. doi:10.1038/nmeth.2474

Chu, Z.-J., Wang, Y.-J., Ying, S.-H., Wang, X.-W., Feng, M.-G., 2016. Genome-Wide Host-Pathogen Interaction Unveiled by Transcriptomic Response of Diamondback Moth to Fungal Infection. PLoS One 11, e0152908. doi:10.1371/journal.pone.0152908

Conesa, A., Gotz, S., Garcia-Gomez, J.M., Terol, J., Talon, M., Robles, M., 2005. Blast2GO: a universal tool for annotation, visualization and analysis in functional genomics research. Bioinformatics 21, 3674-3676. doi:10.1093/bioinformatics/bti610

Contreras-Moreira, B., Vinuesa, P., 2013. GET_ HOMOLOGUES, a versatile software package for scalable and robust microbial pangenome analysis. Appl Environ Microbiol 79, 7696-7701. doi:10.1128/ AEM.02411-13

Danecek, P., Auton, A., Abecasis, G., Albers, C.A., Banks, E., DePristo, M.A., Handsaker, R.E., Lunter, G., Marth, G.T., Sherry, S.T., McVean, G., Durbin, R., 2011. The variant call format and VCFtools. Bioinformatics 27, 2156-2158. doi:10.1093/bioinformatics/btr330

Darbro, J.M., Johnson, P.H., Thomas, M.B., Ritchie, S. a, Kay, B.H., Ryan, P. a, 2012. Effects of Beauveria bassiana on survival, blood-feeding success, and fecundity of Aedes aegypti in laboratory and semi-field conditions. Am J Trop Med Hyg 86, 656-64. doi:10.4269/ ajtmh.2012.11-0455

de Faria, M.R., Wraight, S.P., 2007. Mycoinsecticides and Mycoacaricides: A comprehensive list with worldwide coverage and international classification of formulation types. Biol Control 43, 237-256. doi:10.1016/j. biocontrol.2007.08.001

de Paula, A.R., Brito, E.S., Pereira, C.R., Carrera, M.P., Samuels, R.I., 2008. Susceptibility of adult Aedes aegypti (Diptera: Culicidae) to infection by Metarhizium anisopliae and Beauveria bassiana: prospects for Dengue vector control. Biocontrol Sci Technol 18, 1017-1025. doi:10.1080/09583150802509199

Del Sorbo, G., Schoonbeek, H., De Waard, M. A., 2000. Fungal transporters involved in efflux of natural toxic compounds and fungicides. Fungal Genet Biol 30, 1-15. doi:10.1006/fgbi.2000.1206

English, A.C., Richards, S., Han, Y., Wang, M., Vee, V., Qu, J., Qin, X., Muzny, D.M., Reid, J.G., Worley, K.C., Gibbs, R.A., 2012. Mind the Gap: Upgrading Genomes with Pacific Biosciences RS Long-Read Sequencing Technology. PLoS One 7, 1-12. doi:10.1371/journal. pone. 0047768

Fang, W., Zhang, Y., Yang, X., Zheng, X., Duan, H., Li, Y., Pei, Y., 2004. Agrobacterium tumefaciens-mediated transformation of Beauveria bassiana using an herbicide resistance gene as a selection marker. J Invertebr Pathol 85, 18-24. doi:10.1016/j.jip.2003.12.003

Forlani, L., Pedrini, N., Girotti, J.R., Mijailovsky, S.J., Cardozo, R.M., Gentile, A.G., Hernández-Suárez, C.M., Rabinovich, J.E., Juárez, M.P., 2015. Biological control of the Chagas disease vector Triatoma infestans with the entomopathogenic Fungus Beauveria bassiana combined with an aggregation cue: field, laboratory and mathematical modeling assessment. PLoS Negl Trop Dis 9, e0003778. doi:10.1371/journal.pntd.0003778 


\section{Chapter 4}

Garrison, E., Marth, G., 2012. Haplotype-based variant detection from short-read sequencing. arXiv Prepr arXiv12073907 9. doi:arXiv:1207.3907 [q-bio.GN]

Ghikas, D. V, Kouvelis, V.N., Typas, M. A., 2010. Phylogenetic and biogeographic implications inferred by mitochondrial intergenic region analyses and ITS15.8S-ITS2 of the entomopathogenic fungi Beauveria bassiana and B. brongniartii. BMC Microbiol 10, 174. doi:10.1186/1471-2180-10-174

Grabherr, M.G., Haas, B.J., Yassour, M., Levin, J.Z., Thompson, D.A., Amit, I., Adiconis, X., Fan, L., Raychowdhury, R., Zeng, Q., Chen, Z., Mauceli, E., Hacohen, N., Gnirke, A., Rhind, N., di Palma, F., Birren, B.W., Nusbaum, C., Lindblad-Toh, K., Friedman, N., Regev, A., 2011. Full-length transcriptome assembly from RNA-Seq data without a reference genome. Nat Biotechnol 29, 644-52. doi:10.1038/nbt.1883

Hartelt, K., Wurst, E., Collatz, J., Zimmermann, G., Kleespies, R.G., Oehme, R.M., Kimmig, P., Steidle, J.L.M., Mackenstedt, U., 2008. Biological control of the tick Ixodes ricinus with entomopathogenic fungi and nematodes: Preliminary results from laboratory experiments. Int J Med Microbiol 298, 314-320. doi:10.1016/j.ijmm.2007.10.003

Jones, P., Binns, D., Chang, H.Y., Fraser, M., Li, W., McAnulla, C., McWilliam, H., Maslen, J., Mitchell, A., Nuka, G., Pesseat, S., Quinn, A.F., SangradorVegas, A., Scheremetjew, M., Yong, S.Y., Lopez, R., Hunter, S., 2014. InterProScan 5: Genome-scale protein function classification. Bioinformatics 30, 1236-1240. doi:10.1093/bioinformatics/btu031

Julca, I., Droby, S., Sela, N., Marcet-Houben, M., Gabaldón, T., 2016. Contrasting Genomic Diversity in Two Closely Related Postharvest Pathogens: Penicillium digitatum and Penicillium expansum. Genome Biol Evol 8, 218-227. doi:10.1093/gbe/evv252

Jurka, J., Kapitonov, V. V., Pavlicek, A., Klonowski, P., Kohany, O., Walichiewicz, J., 2005. Repbase Update, a database of eukaryotic repetitive elements. Cytogenet Genome Res 110, 462-467. doi:10.1159/000084979

Kirkland, B.H., Westwood, G.S., Keyhani, N.O., 2004. Pathogenicity of entomopathogenic fungi Beauveria bassiana and Metarhizium anisopliae to Ixodidae tick species Dermacentor variabilis, Rhipicephalus sanguineus, and Ixodes scapularis. J Med Entomol 41, 705-711. doi:10.1603/0022-2585-41.4.705

Korf, I., 2004. Gene finding in novel genomes. BMC Bioinformatics 5, 59. doi:10.1186/1471-2105-5-59

Langmead, B., Trapnell, C., Pop, M., Salzberg, S.L., 2009. Ultrafast and memory-efficient alignment of short DNA sequences to the human genome. Genome Biol 10, R25. doi:10.1186/gb-2009-10-3-r25
Lee, J.-O., Shrestha, B., Sung, G.-H., Han, S.-K., Sung, J.M., 2010. Successful Development of Cordyceps bassiana Stromata from Beauveria bassiana. Mycobiology 38, 13. doi:10.4489/MYCO.2010.38.1.013

Li, H., Durbin, R., 2009. Fast and accurate short read alignment with Burrows-Wheeler transform. Bioinformatics 25, 1754-1760. doi:10.1093/ bioinformatics/btp324

Li, J., Koni, P. a, Ellar, D.J., 1996. Structure of the mosquitocidal delta-endotoxin $\mathrm{CytB}$ from Bacillus thuringiensis sp. kyushuensis and implications for membrane pore formation. J Mol Biol 257, 129-152. doi:10.1006/jmbi.1996.0152

Li, L., 2003. OrthoMCL: Identification of Ortholog Groups for Eukaryotic Genomes. Genome Res 13, 2178-2189. doi:10.1101/gr.1224503

Li, Z., Alves, S.B., Roberts, D.W., Fan, M., Delalibera, I., Tang, J., Lopes, R.B., Faria, M., Rangel, D.E.N., 2010. Biological control of insects in Brazil and China: history, current programs and reasons for their successes using entomopathogenic fungi. Biocontrol Sci Technol 20, 117-136. doi:10.1080/09583150903431665

Medini, D., Donati, C., Tettelin, H., Masignani, V., Rappuoli, R., 2005. The microbial pan-genome. Curr Opin Genet Dev 15, 589-594. doi:10.1016/j. gde.2005.09.006

Meyling, N. V, Lübeck, M., Buckley, E.P., Eilenberg, J., Rehner, S. a, 2009. Community composition, host range and genetic structure of the fungal entomopathogen Beauveria in adjoining agricultural and seminatural habitats. Mol Ecol 18, 1282-93. doi:10.1111/j.1365294X.2009.04095.x

Mnyone, L.L., Lyimo, I.N., Lwetoijera, D.W., Mpingwa, M.W., Nchimbi, N., Hancock, P.A., Russell, T.L., Kirby, M.J., Takken, W., Koenraadt, C.J.M., 2012. Exploiting the behaviour of wild malaria vectors to achieve high infection with fungal biocontrol agents. Malar J 11, 87. doi:10.1186/1475-2875-11-87

Molnár, I., Gibson, D.M., Krasnoff, S.B., 2010. Secondary metabolites from entomopathogenic Hypocrealean fungi. Nat Prod Rep 27, 1241-1275. doi:10.1039/ c001459c

Myers, E.W., 2000. A Whole-Genome Assembly of Drosophila. Science (80- ) 287, 2196-2204. doi:10.1126/ science.287.5461.2196

Petersen, T.N., Brunak, S., von Heijne, G., Nielsen, H., 2011. SignalP 4.0: discriminating signal peptides from transmembrane regions. Nat Methods 8, 785-786. doi:10.1038/nmeth.1701

Price, A.L., Jones, N.C., Pevzner, P.A., 2005. De novo identification of repeat families in large genomes. Bioinformatics 21, 351-358. doi:10.1093/ 


\section{Comparative genomics}

bioinformatics/bti1018

Rehner, S. a, Buckley, E., 2005. A Beauveria phylogeny inferred from nuclear ITS and EF1- $\alpha$ sequences: evidence for cryptic diversification and links to Cordyceps teleomorphs. Mycologia 97, 84-98. doi:10.3852/ mycologia.97.1.84

Rehner, S. a, Posada, F., Buckley, E.P., Infante, F., Castillo, A., Vega, F.E., 2006. Phylogenetic origins of African and Neotropical Beauveria bassiana s.l. pathogens of the coffee berry borer, Hypothenemus hampei. J Invertebr Pathol 93, 11-21. doi:10.1016/j.jip.2006.04.005

Ribeiro, F.J., Przybylski, D., Yin, S., Sharpe, T., Gnerre, S., Abouelleil, A., Berlin, A.M., Montmayeur, A., Shea, T.P., Walker, B.J., Young, S.K., Russ, C., Nusbaum, C., Maccallum, I., Jaffe, D.B., 2012. Finished bacterial genomes from shotgun sequence data. Genome Res 22, 11, 2270-7. doi: 10.1101/gr.141515.112

Schulz, M.H., Zerbino, D.R., Vingron, M., Birney, E., 2012. Oases: robust de novo RNA-seq assembly across the dynamic range of expression levels. Bioinformatics 28, 1086-1092. doi:10.1093/bioinformatics/bts094

Simão, F.A., Waterhouse, R.M., Ioannidis, P., Kriventseva, E. V., Zdobnov, E.M., 2015. BUSCO: Assessing genome assembly and annotation completeness with single-copy orthologs. Bioinformatics 31, 3210-3212. doi:10.1093/ bioinformatics/btv351

Smit, A., Hubley, R., Green, P., 2013. RepeatMasker Open-4.0. 2013-2015. http://www.repeatmasker.org.

Song, T.-T., Zhao, J., Ying, S.-H., Feng, M.-G., 2013. Differential contributions of five $\mathrm{ABC}$ transporters to mutidrug resistance, antioxidion and virulence of Beauveria bassiana, an entomopathogenic fungus. PLoS One 8, e62179. doi:10.1371/journal.pone.0062179

Stamatakis, A., 2006. RAxML-VI-HPC: Maximum likelihood-based phylogenetic analyses with thousands of taxa and mixed models. Bioinformatics 22, 2688-2690. doi:10.1093/bioinformatics/btl446

Stanke, M., Schöffmann, O., Morgenstern, B., Waack, S., 2006. Gene prediction in eukaryotes with a generalized hidden Markov model that uses hints from external sources. BMC Bioinformatics 7, 62. doi:10.1186/14712105-7-62

Sung, J.-M., Lee, J.-O., Humber, R.A., Sung, G.-H., Shrestha, B., 2006. Cordyceps bassiana and Production of Stromata in vitro Showing Beauveria Anamorph in Korea. Mycobiology 34, 1. doi:10.4489/MYCO.2006.34.1.001

Ter-Hovhannisyan, V., Lomsadze, A., Chernoff, Y.O., Borodovsky, M., 2008. Gene prediction in novel fungal genomes using an ab initio algorithm with unsupervised training. Genome Res 18, 1979-1990. doi:10.1101/ gr.081612.108
Thomma, B.P.H.J., Seidl, M.F., Shi-Kunne, X., Cook, D.E., Bolton, M.D., van Kan, J.A.L., Faino, L., 2015. Mind the gap; seven reasons to close fragmented genome assemblies. Fungal Genet Biol 90, 24-30. doi:10.1016/j. fgb.2015.08.010

Trapnell, C., Pachter, L., Salzberg, S.L., 2009. TopHat: Discovering splice junctions with RNASeq. Bioinformatics 25, 1105-1111. doi:10.1093/ bioinformatics/btp 120

Valero-Jiménez, C.A., Debets, A.J.M., van Kan, J.A.L., Schoustra, S.E., Takken, W., Zwaan, B.J., Koenraadt, C.J.M., 2014. Natural variation in virulence of the entomopathogenic fungus Beauveria bassiana against malaria mosquitoes. Malar J 13, 479. doi:10.1186/14752875-13-479

Valero-Jiménez, C.A., Wiegers, H., Zwaan, B.J., Koenraadt, C.J.M., van Kan, J.A.L., 2016. Genes involved in virulence of the entomopathogenic fungus Beauveria bassiana. J Invertebr Pathol 133, 41-49. doi:10.1016/j.jip.2015.11.011

Vernikos, G., Medini, D., Riley, D.R., Tettelin, H., 2015. Ten years of pan-genome analyses. Curr Opin Microbiol 23, 148-54. doi:10.1016/j.mib.2014.11.016

Wang, D., Zhang, Y., Zhang, Z., Zhu, J., Yu, J., 2010. KaKs_Calculator 2.0: A Toolkit Incorporating GammaSeries Methods and Sliding Window Strategies. Genomics, Proteomics Bioinforma 8, 77-80. doi:10.1016/S1672-0229(10)60008-3

Weber, T., Blin, K., Duddela, S., Krug, D., Kim, H.U., Bruccoleri, R., Lee, S.Y., Fischbach, M. a., Müller, R., Wohlleben, W., Breitling, R., Takano, E., Medema, M.H., 2015. antiSMASH 3.0 - a comprehensive resource for the genome mining of biosynthetic gene clusters. Nucleic Acids Res 43, W237-W243. doi:10.1093/nar/gkv437

Xiao, G., Ying, S.-H., Zheng, P., Wang, Z.-L., Zhang, S., Xie, X.-Q., Shang, Y., St Leger, R.J., Zhao, G.-P., Wang, C., Feng, M.-G., 2012. Genomic perspectives on the evolution of fungal entomopathogenicity in Beauveria bassiana. Sci Rep 2, 483. doi:10.1038/srep00483

Zhang, S., Widemann, E., Bernard, G., Lesot, A., Pinot, F., Pedrini, N., Keyhani, N.O., 2012. CYP52X1, representing new cytochrome P450 subfamily, displays fatty acid hydroxylase activity and contributes to virulence and growth on insect cuticular substrates in entomopathogenic fungus Beauveria bassiana. J Biol Chem 287, 13477-86. doi:10.1074/jbc.M111.338947

Zhang, S., Xia, Y.X., Kim, B., Keyhani, N.O., 2011. Two hydrophobins are involved in fungal spore coat rodlet layer assembly and each play distinct roles in surface interactions, development and pathogenesis in the entomopathogenic fungus, Beauveria bassiana. Mol Microbiol 80, 811-826. doi:10.1111/j.13652958.2011.07613.x 


\section{Chapter 4}

Zheng, P., Xia, Y., Xiao, G., Xiong, C., Hu, X., Zhang, S., Zheng, H., Huang, Y., Zhou, Y., Wang, S., Zhao, G.-P., Liu, X., St Leger, R.J., Wang, C., 2011. Genome sequence of the insect pathogenic fungus Cordyceps militaris, a valued traditional Chinese medicine. Genome Biol 12, R116. doi:10.1186/gb-2011-12-11-r116 


\section{Comparative genomics}

\section{Supplementary materials}

Table S1. Summary of the genome quality benchmarks performed with BUSCO.

\begin{tabular}{lcllll}
\hline BUSCO genome benchmarks & Bb1520 & Bb2597 & Bb4305 & Bb5078 & Bb8028 \\
\hline Complete single-copy BUSCOs & $1404(97.64 \%)$ & $1407(97.84 \%)$ & $1397(97.15 \%)$ & $1385(96.31 \%)$ & $1392(96.8 \%)$ \\
Complete duplicated BUSCOs & $78(5.42 \%)$ & $78(5.42 \%)$ & $97(6.75 \%)$ & $76(5.29 \%)$ & $66(4.59 \%)$ \\
Fragmented BUSCOs & $23(1.60 \%)$ & $26(1.81 \%)$ & $34(2.36 \%)$ & $46(3.20 \%)$ & $39(2.71 \%)$ \\
Missing BUSCOs & $11(0.76 \%)$ & $5(0.35 \%)$ & $7(0.49 \%)$ & $7(0.49 \%)$ & $7(0.49 \%)$ \\
\hline
\end{tabular}

Table S2. Summary of the unique genes of Bb8028.

\begin{tabular}{|c|c|c|c|}
\hline Gene name & Description & Length & Signal Peptide \\
\hline BB8028_0001g00810.1 & serine threonine- kinase Sgk2, partial & 790 & $\mathrm{~N}$ \\
\hline BB8028_0001g03220.1 & subtilisin-like serine protease PR1C, partial & 819 & $\mathrm{Y}$ \\
\hline BB8028_0001g03600.1 & hypothetical protein HIM_11279 & 530 & $\mathrm{~N}$ \\
\hline BB8028_0001g03610.1 & ---NA--- & 91 & $\mathrm{~N}$ \\
\hline BB8028_0001g03620.1 & hypothetical protein BBA_06653 & 346 & $\mathrm{~N}$ \\
\hline BB8028_0001g03630.1 & casein kinase I isoform delta & 312 & $\mathrm{~N}$ \\
\hline BB8028_0001g03640.1 & hypothetical protein BBA_10183 & 295 & $\mathrm{~N}$ \\
\hline BB8028_0001g04930.1 & oligopeptide transporter & 796 & $\mathrm{~N}$ \\
\hline BB8028_0001g06000.1 & polyketide synthase & 2521 & $\mathrm{~N}$ \\
\hline BB8028_0001g06010.1 & chalcone synthase B & 381 & $\mathrm{~N}$ \\
\hline BB8028_0001g08740.1 & hypothetical protein BBAD15_g5815 & 776 & Y \\
\hline BB8028_0001g10430.1 & hypothetical protein BBAD15_g11146 & 385 & $\mathrm{~N}$ \\
\hline BB8028_0001g11020.1 & eukaryotic aspartyl protease & 951 & Y \\
\hline BB8028_0001g11820.1 & hypothetical protein FOXG_12643 & 59 & $\mathrm{~N}$ \\
\hline BB8028_0001g11830.1 & isoleucyl-tRNA synthetase & 1070 & $\mathrm{~N}$ \\
\hline BB8028_0001g11840.1 & glutathione-dependent formaldehyde-activating, GFA & 198 & $\mathrm{~N}$ \\
\hline BB8028_0001g11850.1 & secreted aspartic ase, partial & 441 & Y \\
\hline BB8028_0001g11890.1 & integral membrane & 393 & $\mathrm{~N}$ \\
\hline BB8028_0001g11980.1 & phosphotransferase enzyme family & 509 & $\mathrm{~N}$ \\
\hline BB8028_0001g11990.1 & hypothetical protein BBAD15_g12205 & 127 & $\mathrm{~N}$ \\
\hline BB8028_0001g12000.1 & neutral alpha-glucosidase ab precursor & 862 & $\mathrm{~N}$ \\
\hline BB8028_0001g12280.1 & fungal specific transcription factor & 493 & $\mathrm{~N}$ \\
\hline BB8028_0001g12290.1 & FAD-binding domain & 409 & $\mathrm{~N}$ \\
\hline BB8028_0001g12300.1 & C4-dicarboxylate transport mae1 & 428 & $\mathrm{~N}$ \\
\hline BB8028_0001g12310.1 & alpha beta hydrolase & 306 & $\mathrm{~N}$ \\
\hline BB8028_0001g12320.1 & Cyanamide hydratase & 238 & $\mathrm{~N}$ \\
\hline BB8028_0001g12330.1 & MFS transporter, partial & 275 & Y \\
\hline BB8028_0001g12340.1 & MFS transporter, partial & 242 & $\mathrm{~N}$ \\
\hline BB8028_0001g12350.1 & TPA: Zn 2Cys6 transcription factor & 305 & $\mathrm{Y}$ \\
\hline BB8028_0001g12360.1 & Cyanamide hydratase & 244 & $\mathrm{~N}$ \\
\hline
\end{tabular}




\section{Chapter 4}

BB8028_0001g12370.1 Digeranylgeranylglyceryl phosphate synthas

BB8028_0001g12380.1 hypothetical protein BBA_09710

BB8028_0001g12390.1 Heat shock Hsp70, partial

BB8028_0001g12400.1 hypothetical protein BBA_09708

BB8028_0001g12410.1 hypothetical protein BBAD15_g6952

BB8028_0001g12580.1 geranylgeranyl transferase type-2 subunit alpha

BB8028_0001g13210.1 serine threonine kinase

BB8028_0001g14300.1 Checkpoint serine threonine- kinase bub1

BB8028_0001g14310.1 DNA lyase, partial

BB8028_0001g14340.1 ATP phosphoribosyltransferase

BB8028_0001g14420.1 Prefoldin subunit 1

BB8028_0001g14490.1 indoleamine 2,3-dioxygenase

BB8028_0001g14590.1 developmental regulator

BB8028_0001g14600.1 Diacylglycerol O-acyltransferase 2A

BB8028_0001g15640.1 Aerolysin-like toxin, beta complex domain

BB8028_0001g16090.1 Intracellular serine protease

BB8028_0001g16100.1 NACHT and WD40 domain

BB8028_0001g16540.1 hypothetical protein BBAD15_g11212

BB8028_0001g16580.1 arginine deiminase

BB8028_0002g00010.1 nitrate assimilation regulatory nirA

BB8028_0002g00020.1 beta-tubulin

BB8028_0002g00030.1 hypothetical protein BBAD15_g12118

BB8028_0002g00040.1 cAMP-regulated phospho family Igo1, partial

BB8028_0002g00170.1 tat pathway signal sequence

BB8028_0002g00190.1 MFS general substrate transporter

BB8028_0002g00200.1 SAM-dependent methyltransferase

BB8028_0002g00210.1 canalicular multispecific organic anion transporter 1

BB8028_0002g01470.1 thioesterase superfamily

BB8028_0002g01650.1 hypothetical protein BBAD15_g12184

BB8028_0002g03090.1 Ankyrin repeat-containing

$111 \mathrm{~N}$

BB8028_0002g03100.1 Ankyrin repeat domain-containing 50

BB8028_0002g04590.1 hypothetical protein PVAR5_1172

BB8028_0002g04700.1 hypothetical protein BBA_01736

BB8028_0002g05580.1 oxalate decarboxylase family bicupin

BB8028_0002g05590.1 DUF1275 domain

BB8028_0002g05600.1 major facilitator superfamily transporter

BB8028_0002g05610.1 GCN5 family acetyltransferase

BB8028_0002g05620.1 domain-containing, partial

$181 \quad \mathrm{~N}$

$848 \quad \mathrm{~N}$

BB8028_0002g05630.1 NACHT and Ankyrin domain, partial

BB8028_0002g05710.1 serine threonine- kinase Sgk2 


\section{Comparative genomics}

\begin{tabular}{|c|c|c|}
\hline BB8028_0002g05760.1 & myb family transcription factor & 672 \\
\hline BB8028_0002g07440.1 & GRF zinc finger domain containing, partial & 437 \\
\hline BB8028_0002g08400.1 & $\mathrm{ABC}$ multidrug transporter & 1422 \\
\hline BB8028_0002g10070.1 & Ubiquinone biosynthesis coq9 & 257 \\
\hline BB8028_0002g10080.1 & Structural maintenance of chromosomes 6 & 1164 \\
\hline BB8028_0002g10320.1 & $\begin{array}{l}\text { 2-oxoglutarate dehydrogenase E2 component (dihydrolipoamide } \\
\text { succinyltransferase) }\end{array}$ & 433 \\
\hline BB8028_0002g10330.1 & Inheritance of peroxisomes 1 & 537 \\
\hline BB8028_0002g10380.1 & PCI domain-containing & 382 \\
\hline BB8028_0002g10390.1 & GPI transamidase component GAA1 & 599 \\
\hline BB8028_0002g10400.1 & Rhomboid 2 & 264 \\
\hline BB8028_0002g10410.1 & aconitate hydratase & 787 \\
\hline BB8028_0002g10420.1 & $\mathrm{C} 2 \mathrm{H} 2$ finger domain-containing, partial & 120 \\
\hline BB8028_0002g10430.1 & DASH complex, subunit Dam1, partial & 199 \\
\hline BB8028_0002g10440.1 & Beta-lactamase 2 & 281 \\
\hline BB8028_0002g10450.1 & Ribosome assembly 3 & 82 \\
\hline BB8028_0002g10460.1 & DNA repair rhp54 & 799 \\
\hline BB8028_0002g10470.1 & $\mathrm{NADH}$-ubiquinone oxidoreductase $40 \mathrm{kDa}$ subunit & 375 \\
\hline BB8028_0002g10480.1 & ubiquitin carboxyl-terminal hydrolase & 515 \\
\hline BB8028_0002g10650.1 & Importin subunit beta-3 & 1095 \\
\hline BB8028_0002g10660.1 & glutathione-dependent formaldehyde-activating, partial & 138 \\
\hline BB8028_0002g10670.1 & gelsolin & 394 \\
\hline BB8028_0002g12000.1 & multidrug resistant & 568 \\
\hline BB8028_0002g12010.1 & pyruvate kinase & 540 \\
\hline BB8028_0002g12020.1 & methyltransferase type 12 , partial & 144 \\
\hline BB8028_0002g16740.1 & 2-nitropropane dioxygenase & 366 \\
\hline BB8028_0002g16900.1 & beta-tubulin & 463 \\
\hline BB8028_0002g16910.1 & nitrate assimilation regulatory nirA & 742 \\
\hline BB8028_0002g16920.1 & hypothetical protein BBAD15_g11820 & 156 \\
\hline BB8028_0003g00410.1 & $\mathrm{Na}, \mathrm{H}$ K antiporter P-type ATPase & 1108 \\
\hline BB8028_0003g00510.1 & hypothetical protein BBAD15_g9062 & 101 \\
\hline BB8028_0003g00700.1 & lactoylglutathione lyase & 107 \\
\hline BB8028_0003g01320.1 & hypothetical protein BBAD15_g11055 & 356 \\
\hline BB8028_0003g01340.1 & UDP-N-acetylmuramate--L-alanine ligase & 299 \\
\hline BB8028_0003g01350.1 & C6 zinc finger domain & 546 \\
\hline BB8028_0003g01360.1 & CRAL TRIO domain & 584 \\
\hline BB8028_0003g01370.1 & hypothetical protein BBAD15_g11059 & 320 \\
\hline BB8028_0003g01380.1 & alcohol dehydrogenase & 426 \\
\hline BB8028_0003g05350.1 & Pfs, NACHT and Ankyrin domain & 1266 \\
\hline BB8028_0003g12500.1 & hypothetical protein BBAD15_g1557 & 547 \\
\hline BB8028_0003g13490.1 & $\mathrm{C} 2 \mathrm{H} 2$ transcription factor, & 299 \\
\hline BB8028_0003g14330.1 & HEAT repeat & 1555 \\
\hline BB8028_0003g14700.1 & C6 transcription factor, partial & 264 \\
\hline BB8028_0003g15900.1 & peptidase S9B dipeptidylpeptidase IV domain & 806 \\
\hline BB8028_0003g15920.1 & ---NA--- & 121 \\
\hline BB8028_0003g15930.1 & activating transcription factor $7 \mathrm{a}$ & 267 \\
\hline BB8028_0003g15940.1 & ATP synthase beta chain & 512 \\
\hline BB8028_0003g15950.1 & Mitochondrial carrier & 352 \\
\hline
\end{tabular}




\section{Chapter 4}

BB8028_0003g15960.1 hypothetical protein BBA_10106

BB8028_0003g15970.1 interferon-induced GTP-binding Mx

BB8028_0003g15980.1 myb family transcription factor

BB8028_0003g15990.1 hypothetical protein BBA_08326

BB8028_0003g16000.1 fatty acid desaturase

BB8028_0003g16010.1 hypothetical protein BBA_10316

BB8028_0003g16020.1 Ankyrin repeat

BB8028_0003g16030.1 hypothetical protein CCM_03526

BB8028_0003g16040.1 ---NA---

BB8028_0004g00010.1 kinase domain

BB8028_0004g00810.1 hypothetical protein BBAD15_g8288

BB8028_0004g01620.1 subtilisin-like protease, partial

BB8028_0004g01630.1 ---NA---

BB8028_0004g01640.1 peptidase A4 family

BB8028_0004g01650.1 hypothetical protein BBAD15_g10134

BB8028_0004g01660.1 alpha beta hydrolase,

BB8028_0004g01670.1 C6 transcription factor,

BB8028_0004g01680.1 Major facilitator superfamily domain, general substrate transporter, partial

BB8028_0004g01690.1 extracellular phospholipase C, partial

BB8028_0004g01810.1 hypothetical protein GQ26_0710100

BB8028_0004g02320.1 FAD-binding, type 2

BB8028_0004g02330.1 ABC transporter

BB8028_0004g02960.1 DUF159 domain

BB8028_0004g02970.1 DDHD domain-containing

BB8028_0004g03860.1 hypothetical protein BBAD15_g9416

BB8028_0004g04220.1 hypothetical protein BBAD15_g2733

BB8028_0004g04230.1 sulfur metabolite repression control

BB8028_0004g04240.1 ---NA---

BB8028_0004g04250.1 ABC multidrug transporter

BB8028_0004g04260.1 adenylate cyclase, BB8028_0004g04270.1 AChain A, Crystal Structure Of Engineered Northeast Structural Genomics

BB8028_0004g04290.1 ZZ type zinc finger domain-containing

BB8028_0004g04790.1 hypothetical protein MAA_08203

BB8028_0004g05030.1 Pyrroline-5-carboxylate reductase

BB8028_0004g05040.1 hypothetical protein BBAD15_g11461

BB8028_0004g05050.1 dihydrodipicolinate synthetase family

BB8028_0004g05060.1 General amino acid permease AGP2

BB8028_0004g05070.1 delta-1-pyrroline-5-carboxylate dehydrogenase

BB8028_0004g05080.1 FAD dependent oxidoreductase

BB8028_0004g05090.1 Zn 2Cys6 transcription factor

BB8028_0004g05100.1 glutathione-dependent formaldehyde-activating gfa, partial

BB8028_0004g05110.1 Alpha-keto-acid decarboxylase

$111 \quad \mathrm{Y}$

BB8028_0004g05120.1 subtilisin-like serine protease PR1C

$856 \quad \mathrm{Y}$

BB8028_0004g06800.1 dynamin family

$714 \quad \mathrm{~N}$

BB8028_0004g06870.1 ---NA---

$131 \quad \mathrm{~N}$

BB8028_0004g10550.1 hypothetical protein BBAD15_g7215

$631 \mathrm{~N}$ 


\section{Comparative genomics}

\begin{tabular}{|c|c|c|}
\hline BB8028_0004g10810.1 & Cuticle-degrading protease & 397 \\
\hline BB8028_0004g11740.1 & kinase-like domain & 289 \\
\hline BB8028_0004g11970.1 & alpha beta-hydrolase, partial & 360 \\
\hline BB8028_0004g11980.1 & Pesticidal crystal cry $1 \mathrm{Da}$ & 498 \\
\hline BB8028_0004g13420.1 & hypothetical protein ACRE_047590 & 70 \\
\hline BB8028_0004g13430.1 & phloretin hydrolase & 316 \\
\hline BB8028_0004g13440.1 & kinase-like domain & 194 \\
\hline BB8028_0004g13450.1 & phosphotransferase enzyme family & 272 \\
\hline BB8028_0005g00270.1 & glutamate carboxypeptidase & 738 \\
\hline BB8028_0005g00740.1 & metalloprotease 1 & 613 \\
\hline BB8028_0005g01430.1 & Poly [ADP-ribose] polymerase & 761 \\
\hline BB8028_0005g02300.1 & Ankyrin repeat-containing domain, partial & 239 \\
\hline BB8028_0005g02310.1 & U4 tri-snRNP-associated & 269 \\
\hline BB8028_0005g04070.1 & Malic enzyme, NAD-binding, partial & 707 \\
\hline BB8028_0005g04560.1 & C6 finger domain & 409 \\
\hline BB8028_0005g05110.1 & ---NA--- & 127 \\
\hline BB8028_0005g05120.1 & multidrug resistance MDR, & 1434 \\
\hline BB8028_0005g05900.1 & kinase domain-containing, partial & 445 \\
\hline BB8028_0005g05910.1 & Serine cysteine peptidase, trypsin & 359 \\
\hline BB8028_0005g06050.1 & carotenoid ester lipase precursor & 579 \\
\hline BB8028_0005g06600.1 & integral membrane PTH11 & 382 \\
\hline BB8028_0005g07530.1 & QDE-2-interacting, partial & 110 \\
\hline BB8028_0005g08740.1 & chitinase 18-11 & 507 \\
\hline BB8028_0005g09280.1 & t-SNARE affecting a late Golgi compartment 1 & 239 \\
\hline BB8028_0005g09620.1 & family UPF0405, partial & 312 \\
\hline BB8028_0005g09840.1 & hypothetical protein BBA_06207 & 321 \\
\hline BB8028_0005g09850.1 & Phosphoesterase family & 162 \\
\hline BB8028_0005g09860.1 & hypothetical protein BBAD15_g12513 & 182 \\
\hline BB8028_0005g10380.1 & DNA polymerase zeta subunit & 1678 \\
\hline BB8028_0005g10880.1 & nacht and ankyrin domain containing & 1486 \\
\hline BB8028_0005g11130.1 & hypothetical protein BBA_08804 & 84 \\
\hline BB8028_0005g11140.1 & HAD-like domain & 273 \\
\hline BB8028_0005g11150.1 & tetratricopeptide repeat domain-containing & 448 \\
\hline BB8028_0005g11160.1 & SPR1-exo-1,3-beta-glucanase precursor & 432 \\
\hline BB8028_0005g11170.1 & nacht nucleoside triphosphatase & 1347 \\
\hline BB8028_0005g11400.1 & F-box and leucine-rich repeat GRR1 & 207 \\
\hline BB8028_0005g11410.1 & domain-containing & 793 \\
\hline BB8028_0005g11420.1 & hypothetical protein VHEMI07218 & 157 \\
\hline BB8028_0005g11430.1 & hypothetical protein FOCG_16298 & 420 \\
\hline BB8028_0005g11450.1 & hypothetical protein BBA_07475 & 78 \\
\hline BB8028_0005g11440.1 & hypothetical protein BBAD15_g11824 & 296 \\
\hline BB8028_0005g11460.1 & arginine $\mathrm{N}$-methyltransferase 2 , partial & 230 \\
\hline BB8028_0005g11470.1 & restless-like transposase & 648 \\
\hline BB8028_0005g11480.1 & arginine deiminase type- 4 & 824 \\
\hline BB8028_0005g11490.1 & hypothetical protein BBA_09031 & 307 \\
\hline BB8028_0005g11500.1 & metalloprotease & 683 \\
\hline BB8028_0005g11510.1 & hypothetical protein BBA_09031 & 464 \\
\hline
\end{tabular}




\section{Chapter 4}

\begin{tabular}{|c|c|c|}
\hline BB8028_0005g11520.1 & serine threonine- kinase Sgk2 & 776 \\
\hline BB8028_0005g11530.1 & mitochondrial chaperone BCS1 & 489 \\
\hline BB8028_0005g11540.1 & ---NA--- & 78 \\
\hline BB8028_0005g11550.1 & mitochondrial chaperone BCS1 & 225 \\
\hline BB8028_0005g11560.1 & hypothetical protein BBA_09029 & 446 \\
\hline BB8028_0005g11570.1 & Serine threonine- kinase & 570 \\
\hline BB8028_0005g11610.1 & alpha-ribazole-5 -phosphate phosphatase & 253 \\
\hline BB8028_0006g00820.1 & hypothetical protein BBA_09879 & 236 \\
\hline BB8028_0006g00930.1 & bacteriodes thetaiotaomicron symbiotic chitinase & 87 \\
\hline BB8028_0006g01250.1 & glycosyltransferase family 25 & 365 \\
\hline BB8028_0006g01260.1 & hypothetical protein BBAD15_g2621 & 124 \\
\hline BB8028_0006g01270.1 & phosphotransferase family & 468 \\
\hline BB8028_0006g01530.1 & hypothetical protein BBAD15_g12180 & 143 \\
\hline BB8028_0006g01820.1 & Heat-labile enterotoxin IIA, A chain, partial & 313 \\
\hline BB8028_0006g02660.1 & Zn 2Cys6 transcription factor & 715 \\
\hline BB8028_0006g02790.1 & hypothetical protein BBAD15_g8190 & 642 \\
\hline BB8028_0006g02980.1 & prolidase pepP, & 553 \\
\hline BB8028_0006g02990.1 & NACHT and WD domain-containing & 276 \\
\hline BB8028_0006g03220.1 & delta-endotoxin & 205 \\
\hline BB8028_0006g03230.1 & abhydrolase domain-containing & 265 \\
\hline BB8028_0006g03370.1 & very large low complexity & 2806 \\
\hline BB8028_0006g04300.1 & alpha-L-rhamnosidase C & 424 \\
\hline BB8028_0006g04310.1 & peptidylprolyl isomerase & 110 \\
\hline BB8028_0006g04320.1 & integral membrane & 410 \\
\hline BB8028_0006g04330.1 & phosphoribosylamine-glycine ligase & 784 \\
\hline BB8028_0006g06180.1 & ubiquitin-like autophagy Apg12 & 183 \\
\hline BB8028_0006g06190.1 & glutaminyl-tRNA synthetase & 617 \\
\hline BB8028_0006g06250.1 & carbon-nitrogen hydrolase & 320 \\
\hline BB8028_0006g06360.1 & NACHT and Ankyrin domain, partial & 1175 \\
\hline BB8028_0006g06370.1 & hypothetical protein BBA_06219 & 86 \\
\hline BB8028_0006g06420.1 & Zn2Cys6 transcription factor & 487 \\
\hline BB8028_0006g06430.1 & MFS monocarboxylate transporter & 439 \\
\hline BB8028_0006g06480.1 & hypothetical protein BBAD15_g6252 & 289 \\
\hline BB8028_0006g06490.1 & hypothetical protein BBAD15_g6251 & 316 \\
\hline BB8028_0006g06780.1 & hypothetical protein AK830_g12289 & 504 \\
\hline BB8028_0006g10030.1 & cation transporter & 741 \\
\hline BB8028_0006g10830.1 & vegetative incompatibility HET-E-1 & 189 \\
\hline BB8028_0006g10840.1 & zinc finger & 398 \\
\hline BB8028_0006g10850.1 & Kinesin light chain 3 & 439 \\
\hline BB8028_0006g10860.1 & dynamin GTPase, & 722 \\
\hline BB8028_0006g10870.1 & reverse transcriptase & 253 \\
\hline BB8028_0006g10880.1 & ribose-phosphate pyrophosphokinase, & 497 \\
\hline BB8028_0006g10890.1 & metalloprotease & 742 \\
\hline BB8028_0006g10900.1 & negative acting factor & 247 \\
\hline BB8028_0006g10910.1 & aminotransferase class-III & 317 \\
\hline BB8028_0006g10920.1 & kynurenine 3-monooxygenase & 138 \\
\hline BB8028_0006g10930.1 & hypothetical protein FOQG_15976 & 311 \\
\hline
\end{tabular}




\section{Comparative genomics}

\begin{tabular}{|c|c|c|}
\hline BB8028_0006g10940.1 & hypothetical protein FOWG_17095 & 181 \\
\hline BB8028_0006g10950.1 & hypothetical protein FOWG_17094 & 418 \\
\hline BB8028_0006g10960.1 & hypothetical protein FOQG_15975 & 314 \\
\hline BB8028_0006g10970.1 & myb family transcription factor & 491 \\
\hline BB8028_0006g10980.1 & Ribonuclease $\mathrm{H}$ & 720 \\
\hline BB8028_0006g10990.1 & BTB POZ domain & 286 \\
\hline BB8028_0006g11000.1 & ubiquinol-cytochrome-c reductase cytochrome $\mathrm{c} 1$, partial & 231 \\
\hline BB8028_0007g00010.1 & hypothetical protein V491_00904 & 239 \\
\hline BB8028_0007g00330.1 & Interferon-induced GTP-binding Mx & 276 \\
\hline BB8028_0007g00340.1 & SET domain-containing 5 & 420 \\
\hline BB8028_0007g00350.1 & hypothetical protein BBA_10078 & 255 \\
\hline BB8028_0007g00360.1 & heterokaryon incompatibility & 599 \\
\hline BB8028_0007g00370.1 & Endonuclease exonuclease phosphatase, partial & 433 \\
\hline BB8028_0007g00380.1 & glycoside hydrolase family 3 & 792 \\
\hline BB8028_0007g00500.1 & Ankyrin repeat & 261 \\
\hline BB8028_0007g00510.1 & hypothetical protein BBAD15_g12090 & 311 \\
\hline BB8028_0007g00520.1 & hypothetical protein BBAD15_g12091 & 257 \\
\hline BB8028_0007g00530.1 & vegetative incompatibility HET-E-1 & 1351 \\
\hline BB8028_0007g00540.1 & domain-containing, partial & 843 \\
\hline BB8028_0007g00550.1 & heat shock, partial & 575 \\
\hline BB8028_0007g00560.1 & Interferon-induced GTP-binding Mx & 753 \\
\hline BB8028_0007g00570.1 & BTB POZ domain-containing & 361 \\
\hline BB8028_0007g00580.1 & hypothetical protein SETTUDRAFT_36163 & 101 \\
\hline BB8028_0007g00590.1 & zinc finger domain-containing & 231 \\
\hline BB8028_0007g00600.1 & hypothetical protein TOPH_09118 & 152 \\
\hline BB8028_0007g00610.1 & domain-containing & 89 \\
\hline BB8028_0007g00630.1 & NACHT and Ankyrin domain, partial & 128 \\
\hline BB8028_0007g00970.1 & Membrane attack complex component perforin, partial & 739 \\
\hline BB8028_0007g00980.1 & ---NA--- & 68 \\
\hline BB8028_0007g01740.1 & 2-oxoisovalerate dehydrogenase beta subunit & 444 \\
\hline BB8028_0007g01750.1 & exo-beta-1,3-glucanase, partial & 699 \\
\hline BB8028_0007g01760.1 & major facilitator superfamily transporter & 548 \\
\hline BB8028_0007g01770.1 & TRI14, partial & 374 \\
\hline BB8028_0007g02020.1 & ---NA--- & 53 \\
\hline BB8028_0007g02150.1 & trypsin-like protease, partial & 341 \\
\hline BB8028_0007g03650.1 & Concanavalin A-like lectin glucanase & 485 \\
\hline BB8028_0007g03710.1 & peptidase family M20 M25 M40 & 134 \\
\hline BB8028_0007g05070.1 & major facilitator superfamily transporter & 644 \\
\hline BB8028_0007g05350.1 & isoleucyl-tRNA synthetase ,cytoplasmic & 1084 \\
\hline BB8028_0007g05360.1 & hypothetical protein BBA_02890 & 232 \\
\hline BB8028_0007g06370.1 & hypothetical protein BBA_02790 & 613 \\
\hline BB8028_0007g06380.1 & hypothetical protein BBAD15_g10837 & 813 \\
\hline BB8028_0007g06470.1 & copper radical oxidase, partial & 1706 \\
\hline BB8028_0007g06780.1 & methyltransferase & 220 \\
\hline BB8028_0007g06790.1 & HD family hydrolase, & 205 \\
\hline BB8028_0007g06800.1 & methyltransferase & 493 \\
\hline BB8028_0007g06810.1 & multidrug resistance 1 & 1279 \\
\hline
\end{tabular}




\section{Chapter 4}

BB8028_0007g06820.1 DUF636 domain-containing

BB8028_0007g06830.1 hypothetical protein NECHADRAFT_34651

$1072 \mathrm{~N}$

BB8028_0008g00010.1 C6 transcription factor 2, partial

$296 \quad \mathrm{~N}$

BB8028_0008g00020.1 Butirosin biosynthesis, -like

$238 \quad \mathrm{~N}$

BB8028_0008g00030.1 Major facilitator superfamily domain, general substrate transporter, partial

$587 \quad \mathrm{~N}$

BB8028_0008g00040.1 glutathione S-transferase, partial

$249 \quad \mathrm{~N}$

BB8028_0008g00050.1 cytochrome P450 monooxygenase, partial

$471 \quad \mathrm{~N}$

BB8028_0008g00060.1 dimethylallyl transferase, partial

$453 \quad \mathrm{~N}$

BB8028_0008g00070.1 aminotransferase, partial

BB8028_0008g00080.1 non ribosomal peptide synthase, partial

$419 \quad \mathrm{~N}$

$1059 \mathrm{~N}$

BB8028_0008g00090.1 thioredoxin reductase, partial

BB8028_0008g00100.1 peptidase C14

$338 \quad \mathrm{~N}$

BB8028_0008g00110.1 DUF124 domain

$100 \quad \mathrm{~N}$

$250 \quad \mathrm{~N}$

BB8028_0008g00120.1 Aminoglycoside phosphotransferase, partial

$194 \mathrm{~N}$

BB8028_0008g00130.1 Aldo keto reductase

$343 \quad \mathrm{~N}$

BB8028_0008g00140.1 hypothetical protein BBAD15_g6704

$306 \quad \mathrm{~N}$

BB8028_0008g00150.1 fungal specific transcription factor

BB8028_0008g00160.1 NAD-dependent epimerase dehydratase

$351 \quad \mathrm{Y}$

$303 \quad \mathrm{~N}$

BB8028_0008g00170.1 Alpha beta-hydrolase

$405 \quad \mathrm{~N}$

BB8028_0008g00210.1 hypothetical protein BBAD15_g6694

BB8028_0008g00220.1 subtilisin-like protease, partial

426

$606 \quad \mathrm{Y}$

BB8028_0008g00230.1 conidial wall

BB8028_0008g00330.1 hypothetical protein MAA_10155

$131 \quad \mathrm{~N}$

BB8028_0008g00340.1 Acyl-desaturase

$432 \quad \mathrm{Y}$

$429 \quad \mathrm{~N}$

BB8028_0008g00380.1 ABC drug exporter

BB8028_0008g02370.1 FAD binding domain-containing

BB8028_0008g02420.1 vacuolar targeting ATH18

$662 \mathrm{~N}$

BB8028_0008g02430.1 dual specificity phosphatase

BB8028_0008g02520.1 endo-N-acetyl-beta-D-glucosaminidase precursor

$453 \quad \mathrm{~N}$

$703 \quad \mathrm{~N}$

BB8028_0008g02530.1 phosphotransferase enzyme family

$365 \quad \mathrm{Y}$

BB8028_0008g02640.1 hypothetical protein BBAD15_g11010

$409 \quad \mathrm{~N}$

$468 \quad \mathrm{~N}$

BB8028_0009g00010.1 tyrosine phosphatase

$179 \quad \mathrm{~N}$

BB8028_0009g00020.1 heat-labile enterotoxin IIB, A chain

BB8028_0009g00690.1 hypothetical protein BBA_07946

BB8028_0009g01910.1 ---NA---

BB8028_0010g00100.1 hypothetical protein BBA_09215

BB8028_0010g00210.1 hypothetical protein BBAD15_g11500

$503 \quad \mathrm{Y}$

$136 \mathrm{~N}$

$172 \mathrm{~N}$

$83 \quad \mathrm{~N}$

$1104 \quad \mathrm{~N}$

BB8028_0010g00220.1 Glycoside hydrolase, clan GH-D

$293 \quad \mathrm{~N}$

$645 \mathrm{~N}$

BB8028_0010g00420.1 molecular chaperone

BB8028_0010g00430.1 hypothetical protein BBA_09452

$144 \quad \mathrm{~N}$

BB8028_0010g00440.1 hypothetical protein BBA_09453

$251 \quad \mathrm{~N}$

BB8028_0010g00450.1 hypothetical protein BBAD15_g11488

$235 \quad \mathrm{~N}$

BB8028_0010g00460.1 ---NA---

$161 \quad \mathrm{~N}$

BB8028_0010g00470.1 hypothetical protein MBR_09979, partial

$461 \quad \mathrm{~N}$

BB8028_0010g00480.1 Uncharacterized protein MAA_01392

$118 \quad \mathrm{~N}$

BB8028_0010g00490.1 hypothetical protein BBA_04376

$98 \quad \mathrm{Y}$ 


\section{Comparative genomics}

\begin{tabular}{|c|c|c|c|}
\hline BB8028_0010g00510.1 & Heat-labile enterotoxin IIA, A chain, partial & 141 & $\mathrm{~N}$ \\
\hline BB8028_0010g00520.1 & Heat-labile enterotoxin IIA, A chain & 146 & $\mathrm{~N}$ \\
\hline BB8028_0010g00530.1 & metalloprotease 1 , partial & 449 & $\mathrm{~N}$ \\
\hline BB8028_0010g00540.1 & arginine deiminase & 237 & $\mathrm{~N}$ \\
\hline BB8028_0010g00550.1 & siderophore iron transporter & 581 & $\mathrm{~N}$ \\
\hline BB8028_0010g00560.1 & C6 transcription factor & 140 & $\mathrm{~N}$ \\
\hline BB8028_0010g00570.1 & transposase & 280 & $\mathrm{~N}$ \\
\hline BB8028_0010g00590.1 & short-chain dehydrogenase & 260 & $\mathrm{~N}$ \\
\hline BB8028_0010g00600.1 & C6 zinc finger domain-containing & 362 & $\mathrm{~N}$ \\
\hline BB8028_0011g00020.1 & fungal specific transcription factor & 695 & $\mathrm{~N}$ \\
\hline BB8028_0011g00030.1 & hypothetical protein BBAD15_g12121 & 116 & $\mathrm{~N}$ \\
\hline BB8028_0011g00190.1 & FAD binding domain & 366 & $\mathrm{~N}$ \\
\hline BB8028_0011g00200.1 & FAD binding domain & 302 & $\mathrm{~N}$ \\
\hline BB8028_0012g00060.1 & hypothetical protein BBA_09453 & 144 & $\mathrm{~N}$ \\
\hline BB8028_0012g00070.1 & hypothetical protein BBA_09452 & 769 & $\mathrm{~N}$ \\
\hline BB8028_0012g00080.1 & hypothetical protein BBA_09448 & 530 & $\mathrm{~N}$ \\
\hline BB8028_0012g00090.1 & hypothetical protein BBA_09608 & 365 & $\mathrm{~N}$ \\
\hline BB8028_0012g00100.1 & $\mathrm{ABC}$ transporter & 431 & $\mathrm{~N}$ \\
\hline BB8028_0012g00110.1 & ---NA--- & 127 & $\mathrm{~N}$ \\
\hline BB8028_0012g00120.1 & Tfol transposase & 453 & Y \\
\hline BB8028_0012g00130.1 & hypothetical protein BBA_09466 & 98 & Y \\
\hline BB8028_0012g00140.1 & siderophore iron transporter & 205 & $\mathrm{~N}$ \\
\hline BB8028_0012g00150.1 & C6 zinc finger domain-containing & 462 & $\mathrm{~N}$ \\
\hline BB8028_0012g00160.1 & short-chain dehydrogenase & 260 & $\mathrm{~N}$ \\
\hline BB8028_0012g00170.1 & Heat-labile enterotoxin IIA, A chain, partial & 420 & $\mathrm{~N}$ \\
\hline BB8028_0012g00190.1 & hypothetical protein BBA_10082 & 425 & $\mathrm{~N}$ \\
\hline BB8028_0012g00200.1 & TPR domain & 224 & $\mathrm{~N}$ \\
\hline BB8028_0012g00210.1 & cholera enterotoxin subunit A2 & 338 & Y \\
\hline BB8028_0012g00220.1 & isochorismatase family & 184 & $\mathrm{~N}$ \\
\hline BB8028_0012g00230.1 & NADH:flavin oxidoreductase NADH oxidase & 425 & $\mathrm{~N}$ \\
\hline BB8028_0012g00240.1 & pol poly & 1145 & $\mathrm{~N}$ \\
\hline
\end{tabular}




\section{Chapter 4}

Table S3. Summary of the unique genes of Bb8028 that were highly expressed in the infected mosquito library.

\begin{tabular}{|c|c|c|}
\hline Gene name & Description & Length \\
\hline BB8028_0006g03220.1 & delta-endotoxin & 205 \\
\hline BB8028_0006g10880.1 & ribose-phosphate pyrophosphokinase, & 497 \\
\hline BB8028_0002g10410.1 & aconitate hydratase & 787 \\
\hline BB8028_0004g04220.1 & hypothetical protein BBAD15_g2733 & 218 \\
\hline BB8028_0002g04590.1 & hypothetical protein PVAR5_1172 & 111 \\
\hline BB8028_0005g11470.1 & restless-like transposase & 648 \\
\hline BB8028_0005g11160.1 & SPR1-exo-1,3-beta-glucanase precursor & 432 \\
\hline BB8028_0006g10890.1 & metalloprotease & 742 \\
\hline BB8028_0002g05590.1 & DUF1275 domain & 285 \\
\hline BB8028_0002g05600.1 & major facilitator superfamily transporter & 494 \\
\hline BB8028_0003g15920.1 & ---NA--- & 121 \\
\hline BB8028_0003g15930.1 & activating transcription factor $7 \mathrm{a}$ & 267 \\
\hline BB8028_0005g11480.1 & arginine deiminase type- 4 & 824 \\
\hline BB8028_0001g11840.1 & glutathione-dependent formaldehyde-activating, GFA & 198 \\
\hline BB8028_0007g01760.1 & major facilitator superfamily transporter & 548 \\
\hline BB8028_0004g04240.1 & ---NA--- & 110 \\
\hline BB8028_0004g02970.1 & DDHD domain-containing & 937 \\
\hline BB8028_0002g00030.1 & hypothetical protein BBAD15_g12118 & 133 \\
\hline BB8028_0008g00120.1 & Aminoglycoside phosphotransferase, partial & 194 \\
\hline BB8028_0003g16000.1 & fatty acid desaturase & 593 \\
\hline BB8028_0012g00060.1 & hypothetical protein BBA_09453 & 144 \\
\hline BB8028_0007g00010.1 & hypothetical protein V491_00904 & 239 \\
\hline BB8028_0007g00350.1 & hypothetical protein BBA_10078 & 255 \\
\hline BB8028_0007g00600.1 & hypothetical protein TOPH_09118 & 152 \\
\hline BB8028_0001g12360.1 & Cyanamide hydratase & 244 \\
\hline BB8028_0006g01820.1 & Heat-labile enterotoxin IIA, A chain, partial & 313 \\
\hline BB8028_0001g12000.1 & neutral alpha-glucosidase ab precursor & 862 \\
\hline BB8028_0006g06420.1 & Zn2Cys6 transcription factor & 487 \\
\hline BB8028_0002g00170.1 & tat pathway signal sequence & 325 \\
\hline BB8028_0007g03650.1 & Concanavalin A-like lectin glucanase & 485 \\
\hline BB8028_0005g00740.1 & metalloprotease 1 & 613 \\
\hline BB8028_0010g00450.1 & hypothetical protein BBAD15_g11488 & 251 \\
\hline BB8028_0010g00460.1 & ---NA--- & 235 \\
\hline BB8028_0006g01530.1 & hypothetical protein BBAD15_g12180 & 143 \\
\hline BB8028_0010g00510.1 & Heat-labile enterotoxin IIA, A chain, partial & 141 \\
\hline BB8028_0010g00520.1 & Heat-labile enterotoxin IIA, A chain & 146 \\
\hline BB8028_0004g01620.1 & subtilisin-like protease, partial & 751 \\
\hline BB8028_0003g00510.1 & hypothetical protein BBAD15_g9062 & 101 \\
\hline BB8028_0009g00010.1 & tyrosine phosphatase & 179 \\
\hline BB8028_0009g00020.1 & heat-labile enterotoxin IIB, A chain & 503 \\
\hline BB8028_0002g05740.1 & mitochondrial chaperone BCS1 & 501 \\
\hline BB8028_0012g00130.1 & hypothetical protein BBA_09466 & 98 \\
\hline
\end{tabular}




\section{Comparative genomics}

Table S4. Summary of genes of Bb8028 that are under positive selection.

\begin{tabular}{lll}
\hline Gene name & Description & Length \\
\hline BB8028_0001g03320.1 & CAMK kinase & 585 \\
BB8028_0001g10800.1 & calmodulin-binding kinase & 759 \\
BB8028_0001g16160.1 & hypothetical protein BBA_08475 & 221 \\
BB8028_0001g17160.1 & hypothetical protein BBAD15_g9625 & 1972 \\
BB8028_0002g09120.1 & hypothetical protein BBAD15_g3964 & 1046 \\
BB8028_0003g00090.1 & glutenin, high molecular weight subunit DY10-like & 255 \\
BB8028_0003g01850.1 & Allergen V5 Tpx-1 family & 412 \\
BB8028_0003g04460.1 & arabinan endo-1,5-alpha-L-arabinosidase A & 114 \\
BB8028_0003g09370.1 & Suppressor STM1 & 330 \\
BB8028_0004g00290.1 & ABC transporter & 125 \\
BB8028_0004g00890.1 & hypothetical protein BBAD15_g2676 & 586 \\
BB8028_0004g11610.1 & hypothetical protein BBAD15_g7312 & 423 \\
BB8028_0004g11790.1 & nacht and ankyrin domain containing & 1162 \\
BB8028_0004g13810.1 & hypothetical protein BBAD15_g10695 & 189 \\
BB8028_0005g00740.1 & metalloprotease 1 & 613 \\
BB8028_0005g01110.1 & hypothetical protein BBAD15_g10487 & 457 \\
BB8028_0005g04840.1 & small nuclear ribonucleo & 146 \\
BB8028_0005g05910.1 & Serine cysteine peptidase, trypsin & 359 \\
BB8028_0006g00130.1 & Magnesium transporter & 208 \\
\hline
\end{tabular}





\section{Chapter 5}

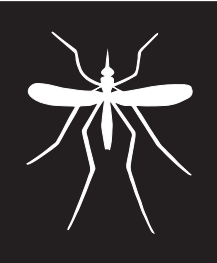

\section{Experimental evolution to increase the efficacy of the entomopathogenic fungus Beauveria bassiana against malaria mosquitoes: effects on mycelial growth and virulence}

Claudio A. Valero-Jiménez ${ }^{1,2}$, Jan A.L. van Kan ${ }^{3}$, Constantianus J.M. Koenraadt ${ }^{2}$, Bas J. Zwaan ${ }^{1, *}$ and Sijmen E. Schoustra ${ }^{1, *}$

${ }^{1}$ Laboratory of Genetics, Wageningen University, P.O. Box 16, 6700 AA Wageningen, The Netherlands ${ }^{2}$ Laboratory of Entomology, Wageningen University, P.O. Box 16, 6700 AA Wageningen, The Netherlands

${ }^{3}$ Laboratory of Phytopathology, Wageningen University, P.O. Box 16, 6700 AA Wageningen, The Netherlands "Shared last author 


\title{
Chapter 5
}

\begin{abstract}
Entomopathogenic fungi such as Beauveria bassiana are currently considered as a potential control agent for malaria mosquitoes. The success of such strategies depends among others on the efficacy of the fungus to kill its hosts. As B. bassiana can use various resources for growth and reproduction, increasing the dependency on mosquitoes as a nutritional source may be instrumental for reaching this goal. Indeed, passage of entomopathogenic fungi through an insect host has been shown to increase its virulence. We evaluated the virulence, fungal outgrowth, mycelial growth rate (MGR), and sporulation rate of two B. bassiana isolates (Bb1520 and Bb8028) that underwent 10 consecutive selection cycles through malaria mosquitoes (Anopheles coluzzii) using an experimental evolution approach. This cycling resulted in an altered capacity of evolved B. Bassiana lineages to grow on different substrates while maintaining the ability to kill insects. Notably, however, there were no significant changes in virulence or speed of outgrowth when comparing the evolved lineages against their un-evolved ancestors. Importantly, evolved lineages from Bb1520 showed a decrease in MGR when grown on three different media that differed in their carbon source, but no change was observed in sporulation rate. In contrast, evolved lineages from Bb8028 showed a faster MGR on trehalose- and glucose-containing media and sporulation rate was higher when grown on trehalose. These results suggest that fungal growth and sporulation evolved through successive and exclusive use of an insect host as a nutritional resource. We discuss the results in the light of biocontrol and provide suggestions to increase fungal virulence.
\end{abstract}

\section{Keywords}

Beauveria bassiana; experimental evolution; entomopathogenic fungi; virulence; mycelial growth rate; malaria mosquitoes 


\section{Experimental evolution}

\section{Introduction}

Beauveria bassiana is a cosmopolitan ascomycete fungus which is able to live as a saprophyte in the soil, as an endophyte in plants, and as an entomopathogen affecting a broad range of arthropods (Rehner et al., 2011). It is used as a biocontrol agent for different arthropods that are a pest in agriculture such as whiteflies, aphids, beetles, and locusts (Li et al., 2010). The use of $B$. bassiana is currently investigated for the potential control of malaria mosquitoes (Kanzok and Jacobs-Lorena, 2006; Knols et al., 2010; Mnyone et al., 2012; Thomas and Read, 2007). There is a pressing need to develop novel tools to control malaria mosquitoes (Anopheles spp.), due to the rapid development of insecticide resistance in mosquito populations (Ranson and Lissenden, 2016). Entomopathogenic fungi could be a suitable alternative tool because they are also effective against insecticide-resistant mosquitoes (Farenhorst et al., 2009; Howard et al., 2011, 2010). Furthermore, fungal biopesticides induce a late-acting mortality, in which adult mosquitoes die in 7-10 days. This is before the malaria parasite can be transmitted (Thomas and Read, 2007), but after reproduction of the mosquitoes thereby reducing the effectiveness of selection for resistance of mosquitoes to fungal infection since this selection pressure only starts towards the end of life (in a so-called selection shadow). Fungal infection also causes sub-lethal effects on the mosquitoes including reduced host-seeking behaviour and feeding propensity (Blanford et al., 2011; Scholte et al., 2006), which potentially further reduces malaria parasite transmission.

Currently, one of the major hurdles that affect the usage of entomopathogenic fungi in field applications is their relatively poor and unpredictable performance compared to traditional insecticides. This is partly caused by variations in environmental factors such as temperature, humidity, and UV exposure that affect the fungal viability, as well as persistence, and virulence (Jaronski, 2010). Particularly, while chemical insecticides have a long residual effect, fungal biological control agents need to be applied more frequently which requires more efficient and complicated logistics, and mass production of biological material. To overcome some of these issues, genetic modification has been implemented to increase the ability to kill insects and tolerate adverse environmental conditions. For example, overexpression of the chitinase gene Bbchit1 of $B$. bassiana resulted in increased infection efficiency and accelerated fungal infection (Fang et al., 2005). Expression of a gene encoding the insect-specific scorpion neurotoxin AAIT in a $B$. bassiana isolate was shown to enhance virulence compared to the wild type isolate (Lu et al., 2008). Fungal tolerance to oxidative stress has been achieved by overexpression of an endogenous superoxide dismutase that is involved in the detoxification of reactive oxygen species (Xie et al., 2010). Other approaches have focused on targeting host-specific molecules to interfere with normal insect development, e.g., by using an isolate expressing a trypsin modulating oostatic factor which showed increased virulence against An. gambiae (Kamareddine et al., 2013). 


\section{Chapter 5}

Although genetic modification for enhancement of virulence has shown encouraging results, field application of genetically modified organisms is associated with regulatory and societal issues that currently limit its usefulness (Koenraadt and Takken, 2011). Therefore, exploiting technical and biological (non-GMO) resources is a feasible and a realistic alternative to increase the efficacy of fungal biological control agents. In this regard, various efforts have been made to improve the delivery of spores to the insect, including UV-protectants and humidity stabilizers, and using different growth substrates that increase sporulation, virulence, and stress resistance (Jaronski, 2010). Increased tolerance to high temperature has been achieved for the fungus Metarhizium anisopliae through directed evolution using continuous culture (de Crecy et al., 2009). Viaud et al. (1998) created somatic hybrids from B. bassiana and B. sulfurescens that were more virulent and stable after cycling through an insect. By using artificial selection, a commercial isolate of $B$. bassiana became resistant to fungicides without reducing its virulence (Shapiro-Ilan et al., 2002). The use of UV irradiation has also been used to obtain mutants that were 2-deoxy-D-glucose resistant and that showed increased virulence (Robledo-Monterrubio et al., 2009).

To date, there are no reports on the exploitation of spontaneous genetic changes combined with natural selection to increase the efficiency of B. bassiana as a biological control agent. When performed under controlled conditions in the laboratory this approach is called experimental evolution, and it can in principle be used to alter fungal traits through spontaneous mutation and natural selection (Schoustra et al., 2012). Furthermore, the use of experimental evolution is potentially an effective tool to identify genes and mechanisms underpinning natural variation in virulence (Valero-Jiménez et al., 2016). In this study, we evaluated, (1) whether B. bassiana is suitable for experimental evolution by assaying the potential to evolutionary changes of growth parameters, and, (2) whether it is possible to select for higher fungal virulence against mosquitoes. To this end, we used an experimental evolution approach in which B. bassiana was forced to use malaria mosquitoes as the sole nutritional resource for 10 consecutive selection cycles of around 80 fungal mitotic generations each (Figure 1). By comparing evolved lineages with their respective ancestors, we examined if and how the phenotypic changes, which occurred using a natural selection regime, altered the virulence of B. bassiana towards mosquitoes. Specifically, we first test whether our selection protocol allows for fixation of genetic changes in evolving fungal lineages by measuring changes in phenotypic characteristics such as mycelial growth rate and sporulation rate. Secondly, we test our evolved lineages for changes in fungal virulence against mosquitoes and discuss our results in the light of biocontrol strategies. 


\section{Experimental evolution}

\section{Materials and methods}

\section{Mosquito rearing and fungal isolates}

Anopheles coluzzii mosquitoes used in the experiments originated from Suakoko, Liberia, and were reared in Wageningen University since 1989 under standard laboratory conditions. Adults were maintained in climate controlled rooms $\left(27 \pm 1^{\circ} \mathrm{C}, 80 \pm 10 \% \mathrm{RH}\right.$ and a $\left.12 \mathrm{~h} \mathrm{~L}: \mathrm{D}\right)$ and fed ad libitum on a $6 \%$ glucose solution. The fungal isolates used were Beauveria bassiana ARSEF 1520 (Bb1520) and ARSEF 8028 (Bb8028), obtained from the USDA-ARS Collection of Entomopathogenic Fungal Cultures (ARSEF). Initially the fungus was grown on Sabouraud Dextrose Agar with $1 \%$ yeast extract (SDAY) for 14 days at $27^{\circ} \mathrm{C}$ and spores were harvested with a $0.05 \%$ Tween 80 solution to make a spore suspension. This suspension was kept at $-80^{\circ} \mathrm{C}$ until use.

\section{Initial bioassay}

Two fungal isolates were subjected to experimental evolution and were chosen based on their natural difference in virulence, as established in a previous study (Valero-Jiménez et al., 2014). Isolate Bb8028 is highly lethal, while isolate Bb1520 is considered as having intermediate lethality. The initial bioassay was done as described in (Valero-Jiménez et al., 2014). In brief, for each isolate, a fungal spore solution was applied to an A4-sized paper one day before exposure and left to dry in a fume hood. Spore viability was checked on SDAY plates after 18-20 h at $27^{\circ} \mathrm{C}$, and spores with a detectable germ tube were considered viable. The coated papers were placed in PVC tubes $(15 \mathrm{~cm}$ height and $8 \mathrm{~cm}$ diameter) that were sealed with cling film on both ends and five replicate PVC tubes were used for each isolate tested. As a control, papers were coated with Shellsol $T^{\oplus}$ oil only. Forty An. coluzzii female mosquitoes (3-5 days old) were transferred to each PVC tube with the coated papers and exposed for three hours. Subsequently, mosquitoes were transferred to clean buckets $(25 \mathrm{~cm}$ height and $20 \mathrm{~cm}$ diameter), which were sealed with a nylon sock. All buckets were kept in a climate controlled chamber $\left(27 \pm 1^{\circ} \mathrm{C}, 80\right.$ $\pm 10 \% \mathrm{RH}, 12 \mathrm{~h} \mathrm{~L}$ :D). Mosquitoes were fed ad libitum with a $6 \%$ glucose solution on a cotton plug placed on top of the nylon stocking. Mortality was checked daily for 14 days and fungal infection of dead mosquitoes was checked by dipping them for $5 \mathrm{sec}$ in $70 \%$ ethanol, incubating them on moist filter paper in sealed petri dishes at $27^{\circ} \mathrm{C}$ for seven to ten days, and inspecting them for visible fungal outgrowth.

\section{Experimental evolution set-up}

The experimental evolution set-up was based on the dead mosquitoes from the initial bioassay, in which fungal spores that grew out of dead mosquitoes (hereafter called sporulating mosquitoes) were used as an inoculum to infect a new batch of mosquitoes (Figure 1). A petri dish without 


\section{Chapter 5}

a lid was placed at the bottom of a bucket, which was then closed with a nylon sock. Each petri dish contained twelve sporulating mosquitoes that died 4-6 days after exposure and that were previously incubated for 8-9 days. Forty An. coluzzii female mosquitoes (3-5 days old) were transferred to the bucket and exposed to sporulating mosquitoes for $24 \mathrm{~h}$. After this period, mosquitoes were transferred to a clean bucket (without sporulating mosquitoes), and mortality was recorded daily for eight days. There were five replicated evolving lineages per starting ancestor, and each replicate was considered as a separate evolution lineage. As a non-evolving control, three replicates were exposed to an empty petri dish to provide a baseline for mortality in each round of selection. All buckets were kept in a climate controlled chamber $\left(27 \pm 1^{\circ} \mathrm{C}, 80 \pm 10 \% \mathrm{RH}, 12\right.$ h L:D) and mosquitoes were fed ad libitum with a $6 \%$ glucose solution on a cotton plug. This experimental procedure lasted two weeks and was repeated for ten selection cycles in total. After each cycle, dead sporulating mosquitoes that had died 4-6 days after infection were put on SDAY plates for 14 days at $27^{\circ} \mathrm{C}$. Spores were harvested with a $0.05 \%$ Tween 80 solution to make a spore suspension and then kept at $-80^{\circ} \mathrm{C}$ to have a fossil record of each cycle. This fossil record was used in a final bioassay that simultaneously tested the virulence of fungal spores from the 10th cycle (evolved) against the spores of the ancestors at the same time and using the same batch of mosquitoes. This was done to minimize variation in the rearing and experimental procedures during the time of this experiment. The final bioassay was conducted in a similar way to the initial bioassay, and two evolutionary lineages were chosen for each ancestral isolate.

\section{Phenotypic characterization of evolved fungal lineages}

The mycelial growth rate (MGR) and the sporulation rate (SR) of all lineages derived from each ancestral isolate were measured and compared against the ancestor of each lineage. The MGR and SR were measured on four different media that differed in carbon source. The fungus was grown on Saboraud Dextrose Agar with 1\% yeast extract (SDAY) containing 40 g glucose, $10 \mathrm{~g}$ Peptone, $10 \mathrm{~g}$ yeast extract, $15 \mathrm{~g}$ agar in $1 \mathrm{l}$ of distilled water; Trehalose medium containing 40 $\mathrm{g}$ trehalose, $10 \mathrm{~g}$ Peptone, $10 \mathrm{~g}$ yeast extract, $15 \mathrm{~g}$ agar in $1 \mathrm{l}$ of distilled water; Insect medium containing minimal medium $\left(0.4 \mathrm{~g} \mathrm{KH}_{2} \mathrm{PO}_{4}, 1.8 \mathrm{~g} \mathrm{Na}_{2} \mathrm{HPO}_{4} \cdot 2 \mathrm{H}_{2} \mathrm{O}, 0.6 \mathrm{~g} \mathrm{MgSO} \cdot 7 \mathrm{H}_{2} \mathrm{O}, 1 \mathrm{~g}\right.$ $\mathrm{KCL}, 0.7 \mathrm{~g} \mathrm{NH}_{4} \mathrm{NO}_{3}$ ) complemented with $20 \mathrm{~g}$ of crushed dead pupae of silkworms and $15 \mathrm{~g}$ agar in 11 of distilled water; Hexadecane medium containing minimal medium with n-hexadecane (1 $\mathrm{ml}$ of a $20 \%$ solution diluted in hexane) as carbon source and $15 \mathrm{~g}$ agar in 11 of distilled water. The media were chosen as representation of the conditions that the fungus was exposed to during the experimental evolution. SDAY was used as the reference medium for culturing of B. bassiana. Trehalose was chosen since it is the main disaccharide in the insect haemolymph. Whole dead insects were chosen because they simulate the complexity of nutrients found on mosquitoes, and hexadecane was selected because is one of the main hydrocarbons present on the outer cuticle 
40x गर

A
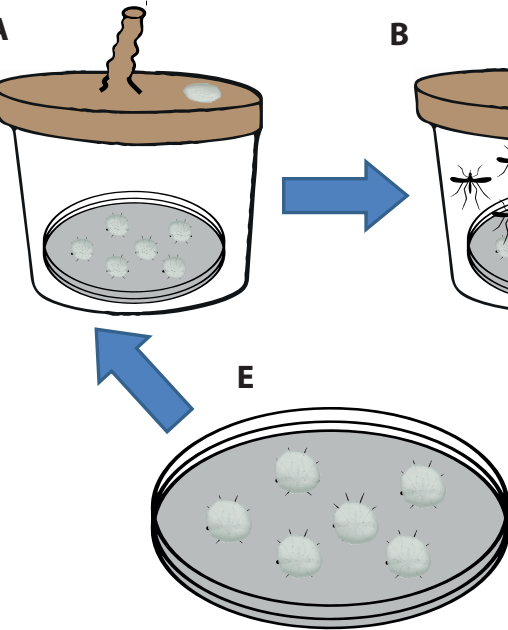
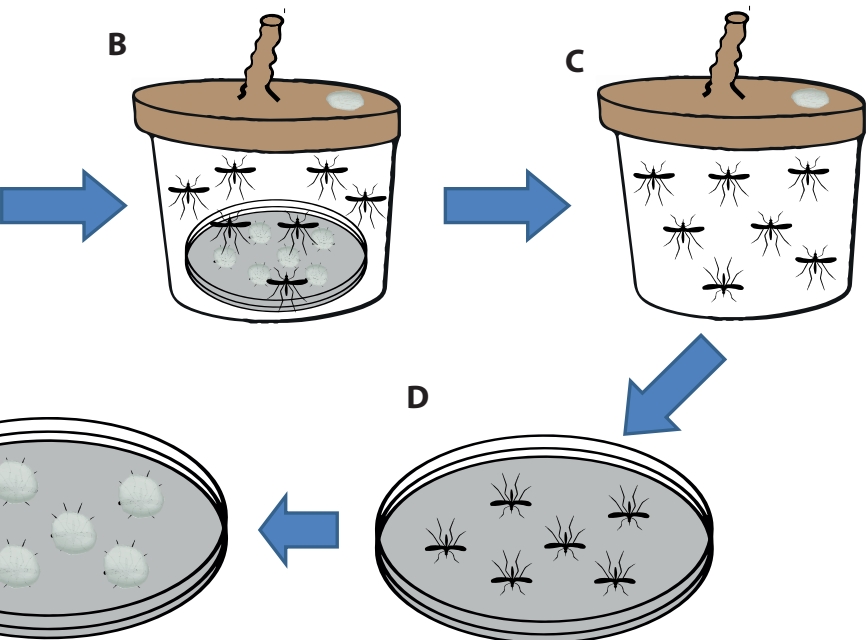

Figure 1. Set-up of the experimental evolution assays that were used to evolve different Beauveria bassiana isolates using Anopheles coluzzii as the sole nutritional resource for 10 consecutive selection cycles. For every selection cycle (A), 40 uninfected An. coluzzii were exposed to twelve sporulating conspecific mosquitoes (mosquitoes that died of a fungal infection) in a bucket and then closed with a nylon sock. The mosquitoes were kept for $24 \mathrm{~h}(\mathrm{~B})$, in order to get a new infection. After this period, mosquitoes were transferred to a clean bucket (without sporulating mosquitoes) and mortality was recorded for eight days (C). Mosquitoes that died after four to six days were transferred to a petri dish with moist filter paper and incubated at $27^{\circ} \mathrm{C}$ (D). After eight to nine days, dead mosquitoes were fully covered with fungal spores, and were used as inoculum for the next cycle (E). There were five replicated evolving lineages per starting ancestor, and each replicate was considered as a separate evolutionary lineage. All buckets were kept in a climate controlled chamber $\left(27 \pm 1^{\circ} \mathrm{C}, 80 \pm 10 \% \mathrm{RH}, 12 \mathrm{~h}\right.$ L:D). Mosquitoes were fed ad libitum with a $6 \%$ glucose solution on a cotton plug provided on top of the nylon stocking.

of insects. Spores were grown on SDAY petri dishes for four days and later agar plugs were used to inoculate the plates. Three plates were inoculated for each combination of replicate, isolate and medium. After two weeks, mycelial growth rate (MGR) was determined by measuring the colony diameter (in $\mathrm{mm}$ ) in two perpendicular directions (Schoustra et al., 2012). To determine sporulation rate (SR), spores were harvested from a 3-weeks-old culture with $0.05 \%$ Tween 80 solution and serially diluted on a 96-well plate. Three dilutions were plated for each isolate/ medium on SDAY plates and incubated for $72 \mathrm{~h}$ at $27^{\circ} \mathrm{C}$. Colonies were counted and the cfu/ $\mathrm{ml}$ was determined.

\section{Quantification of fungal growth rate on dead mosquitoes}

To determine whether there was a change in growth rate after a mosquito died between the evolved lineages and their ancestors, we conducted an experiment to quantify the amount of fungal genomes present at three time points, based on the method developed by Bell et al. 


\section{Chapter 5}

(2009). Lineage four of isolate Bb8028 from the 10th cycle and spores of Bb8028 from the initial bioassay were used. Mosquitoes were infected following a similar procedure as the initial bioassay. Infected mosquitoes which died from days 5-7 were incubated at $27^{\circ} \mathrm{C}$ as described before. A subset of mosquitoes were removed from the petri dish after two, four, or six days of incubation and frozen at $-80{ }^{\circ} \mathrm{C}$. DNA isolation was done on each mosquito using the standard protocol from DNAeasy plant mini kit (Qiagen) and following the recommendations from Bell et al. (2009). Real-time quantitative PCR (RT-qPCR) was performed using specific primers that only amplify DNA from Beauveria bassiana (Forward: GCCGGCCCTGAAATGG; Reverse: R: GATTCGAGGTCAACGTTCAGAAG). Absolute quantification of fungal load was determined by comparing $\mathrm{Ct}$ values against a standard curve, generated from dilutions of DNA aliquots from isolate Bb8028 starting from $10^{8}$ conidia. Three replicates of each DNA standard $\left(10^{3}-10^{7}\right.$ conidia) were included in each RT-qPCR run.

\section{Statistical analysis}

Mosquito survival was analysed using Kaplan-Meier survival analysis in SPSS (v.22) with significant differences between different isolates or ancestor/evolved lineages estimated using a Log Rank Test (Valero-Jiménez et al., 2014). To detect differences between the numbers of fungal genomes of infected mosquitoes, a two-way ANOVA test was used in R (3.2.0). One-way ANOVA was conducted to detect differences in mycelial growth rate and sporulation rate in $\mathrm{R}$ (3.2.0), with the sporulation data being Log transformed before analysis. Differences between ancestor and evolved lineages were calculated with a Tukey's HSD post-hoc test.

\section{Results}

\section{Experimental evolution}

Fungal isolates $\mathrm{Bb} 1520$ and $\mathrm{Bb} 8028$ were used to set up the evolution experiment of 10 successive selection cycles through female An. coluzzii as a host with five replicate evolving lineages per starting isolate. The virulence of each ancestral isolate was corroborated in an initial bioassay (Figure 2), and the spores that grew out of the dead mosquitoes served as starting material for experimental evolution. The mosquito survival differed depending on the isolate used $\left(\mathrm{x}_{2}\right.$ $=48.73, \mathrm{p}<0.001$ ), in which isolate Bb8028 (high lethality) was significantly more virulent than isolate Bb1520 (intermediate lethality) in agreement with our previously published results (Valero-Jiménez et al., 2014).

\section{Phenotypic characterization of evolved fungal lineages}

The ancestral and evolved lineages of isolates Bb1520 and Bb8028 were grown on four media that mainly differed in the carbon source, and served as conditions representing the selection 


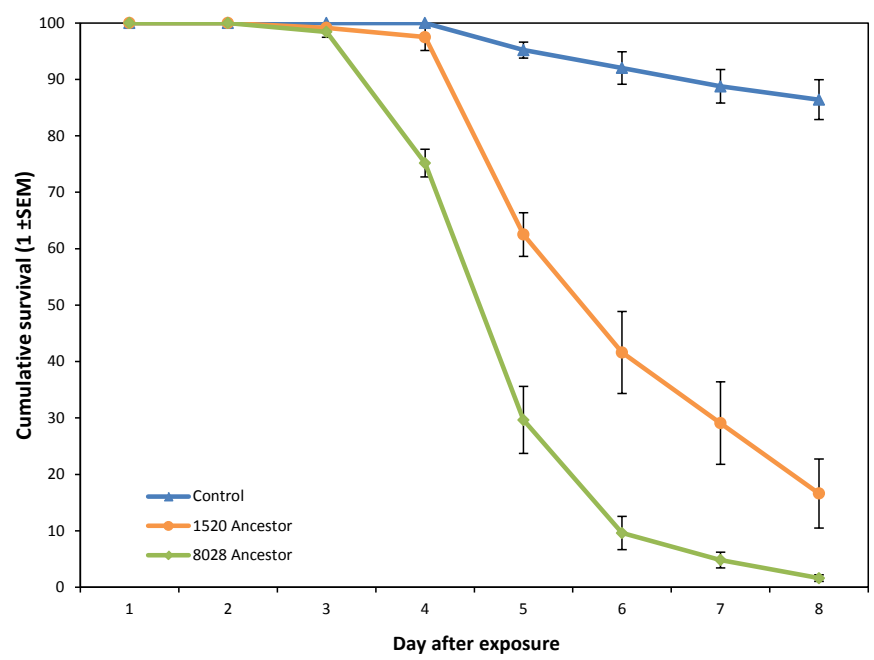

Figure 2. Daily proportional survival of Anopheles coluzzii infected with spores of different Beauveria bassiana isolates. In the control treatments, mosquitoes were exposed to only Shellsol $\mathrm{T}^{\oplus}$ oil. Isolates Bb1520 and Bb8028 were used in the initial bioassay as a starting point for the experimental evolution. Data show means \pm SEM from three replicates of 40 female mosquitoes.

environment (the insect cuticle, the insect haemolymph, the dead mosquitoes). The mycelial growth rate and sporulation rate were measured to test whether phenotypic changes had occurred during this period of experimental evolution. In the SDAY medium (Figure 3A), evolved lineages of Bb1520 (intermediate lethality) and Bb8028 (high lethality) significantly differed in mycelial growth (MGR) compared to their respective ancestors $\left(F_{3,51}=150.5, p<0.001\right)$, although the direction was opposite. The evolved Bb1520 had a significantly lower MGR, while the evolved Bb8028 had a considerably higher MGR than its ancestor. In the trehalose medium (Figure 3B), the evolved Bb8028 had a significantly higher MGR than its ancestor $\left(\mathrm{F}_{3,48}=316.1, \mathrm{p}<0.001\right)$. On the other hand, when the fungus was grown on the insect medium (Figure 3C), both evolved isolates had a significantly lower MGR than their respective ancestors $\left(F_{3,49}=245.3, p<0.001\right)$. Similarly, when grown on hexadecane as a carbon source (Figure 3D), both evolved isolates performed worse than their ancestor with regards to $\operatorname{MGR}\left(\mathrm{F}_{3,55}=200.2, \mathrm{p}<0.001\right)$. For isolate $\mathrm{Bb} 1520$, the MGR of the ancestor was relatively high (as compared to the ancestor of Bb8028) when growing in SDAY and trehalose medium, which could have limited the improvement in growth.

The sporulation rate (SR) varied significantly between the isolates grown on SDAY medium $\left(\mathrm{F}_{3,14}=4.388, \mathrm{p}=0.026\right.$, Figure $\left.4 \mathrm{~A}\right)$, although the evolved lineages did not differ from their respective ancestor $(\mathrm{p}=0.999$ and $\mathrm{p}=0.065$ for Bb1520 and Bb8028, respectively). The evolved lineages of Bb1520 on average had a significantly lower SR than their ancestor when grown 


\section{Chapter 5}
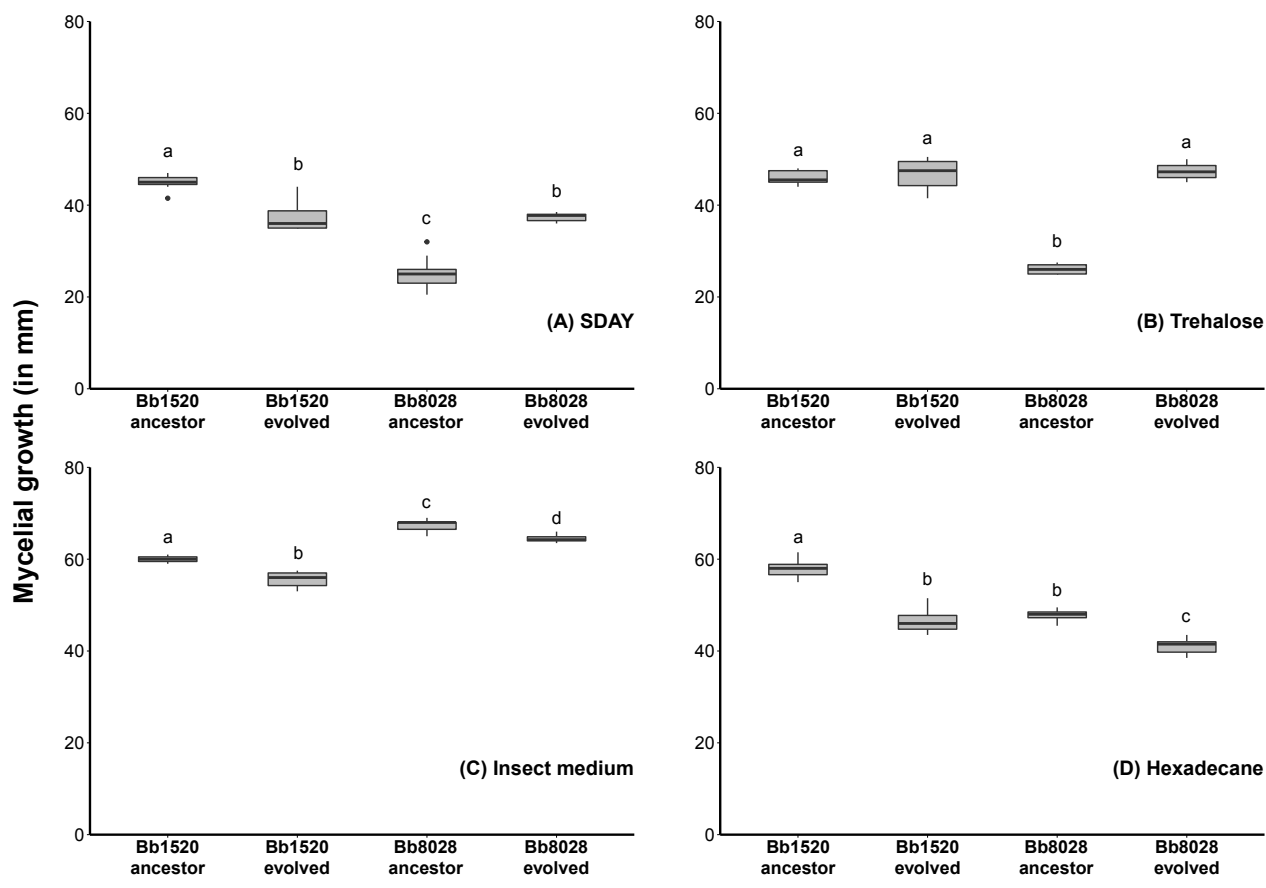

Figure 3. Box plot graphs representing the mycelial growth rate of five replicates of Beauveria bassiana grown on four different media that differ in the carbon source: SDAY (A), trehalose (B), insect medium (C) and hexadecane (D). The box plots shows the median (black line), the box boundaries mark the 25th and 75th quartiles, and the whiskers show the maximum and minimum values, while the dot represents the outliers. Treatments with the same lowercase letter are not significantly different in post hoc comparisons (Tukey's HSD, $\mathrm{p}=0.05$ ).

on trehalose (Figure $4 \mathrm{~B}$ ), while the evolved lineages of Bb8028 on average had a considerably higher $\mathrm{SR}$ than their ancestor $\left(\mathrm{F}_{3,15}=12.6, \mathrm{p}<0.001\right)$. The variation in $\mathrm{SR}$ of the isolates grown on insect medium (Figure $4 \mathrm{C}$ ) was not significant $\left(\mathrm{F}_{3,16}=1.178, \mathrm{p}=0.349\right)$. While there were significant differences on $\mathrm{SR}$ when all isolates were grown on hexadecane medium $\left(\mathrm{F}_{3,15}=3.942\right.$, $\mathrm{p}=0.0294$, Figure 4D), the evolved lineages did not differ from their respective ancestor. To further investigate differences between ancestral and evolved isolates of Bb1520 and Bb8028, fungal growth was measured during growth on 35 different media differing in carbon source (supporting information, Data S1). The MGR of the evolved Bb1520 lineage (Figure S1A) was lower in 26 of the 35 media when compared to its ancestor, and for the remaining nine carbon sources no discernible difference was observed. In contrast, the evolved lineage of Bb8028 (Figure S1B) had a faster growth in 11 different media, eight with a lower growth, and 16 without a discernible difference in growth, when compared to its ancestor. 

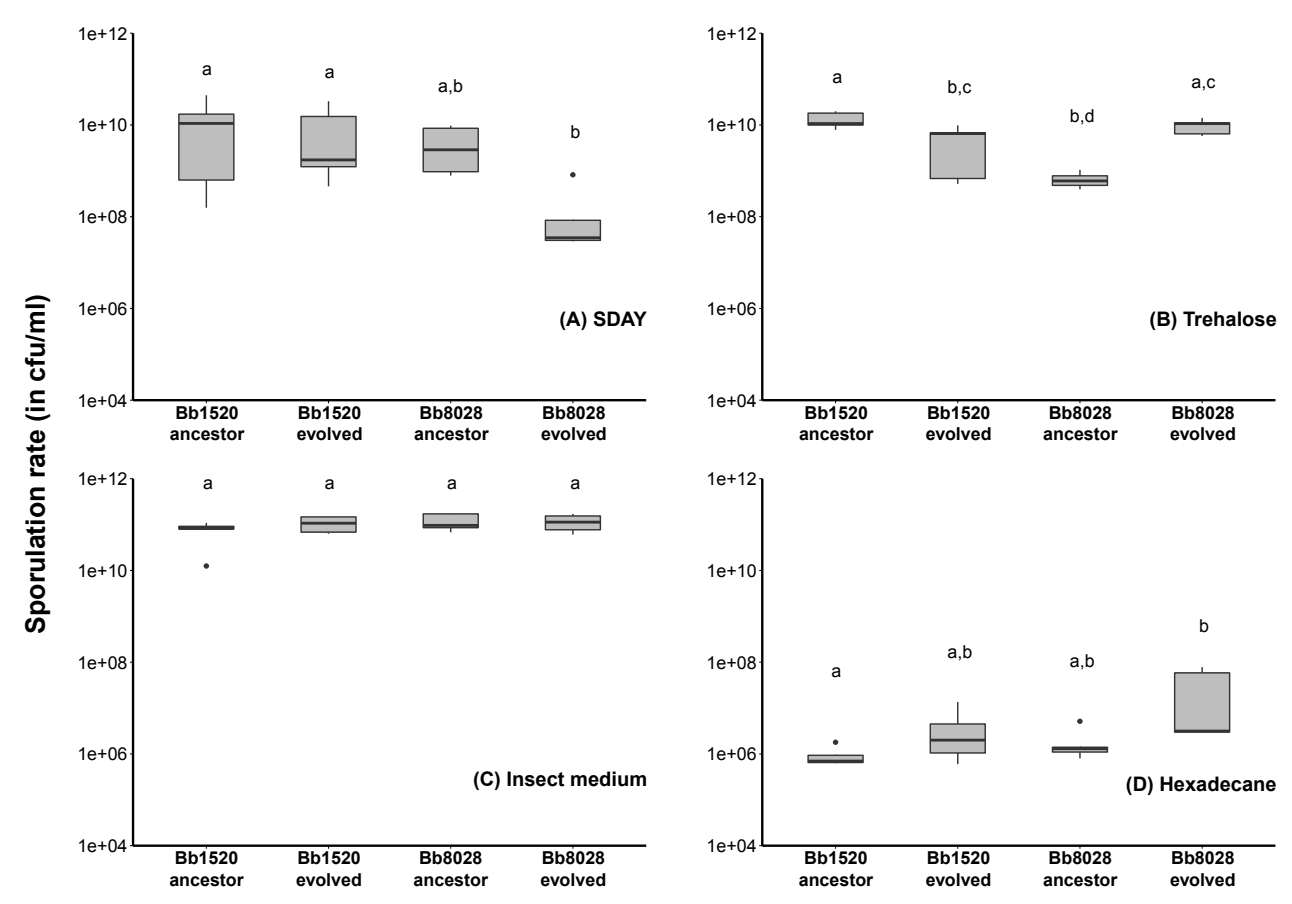

Figure 4. Box plot graphs representing the sporulation rate of five replicates of Beauveria bassiana grown on four different media that differ in the carbon source: SDAY (A), trehalose (B), insect medium (C) and hexadecane (D). The box plots shows the median (black line), the box boundaries mark the 25th and 75th quartiles, and the whiskers show the maximum and minimum values, while the dot represents the outliers. Treatments with the same lowercase letter are not significantly different in post hoc comparisons (Tukey's HSD, $\mathrm{p}=0.05$ ).

\section{Mortality rate}

During the course of the evolution experiment, mosquito survival was measured in each selection cycle, and for both isolates the median survival time varied along the 10 cycles of the experiment. Mosquitoes infected with isolate Bb8028 (Figure 5A) and isolate Bb1520 (Figure 5B) had a median survival time from 5 to 7 days, and 6 to 8 days, respectively. All the experimental evolution replicates for isolate $\mathrm{Bb} 1520$ (intermediate lethality) resulted in a similar mortality rate for the mosquitoes across the 10 selection cycles $\left(x_{2}=7.64\right.$, d.f. $\left.=4, p=0.106\right)$. In addition, all the experimental evolution replicates for isolate Bb8028 (high lethality) behaved similarly, except replicate $4\left(x_{2}=24.97\right.$, d.f. $\left.=4, p<0.001\right)$, which had a significantly higher virulence across the 10 selection cycles. To further test whether there was a change in the virulence of the fungal isolates throughout the experimental evolution, the ancestor (spores from the initial bioassay) were tested against two evolved lineages (spores from the 10th cycle) at the same time. Because replicate 4 from isolate Bb8028 was significantly different from the other replicates, it was included in this final assay while the other lineages were chosen at random. Replicates 2 and 


\section{Chapter 5}

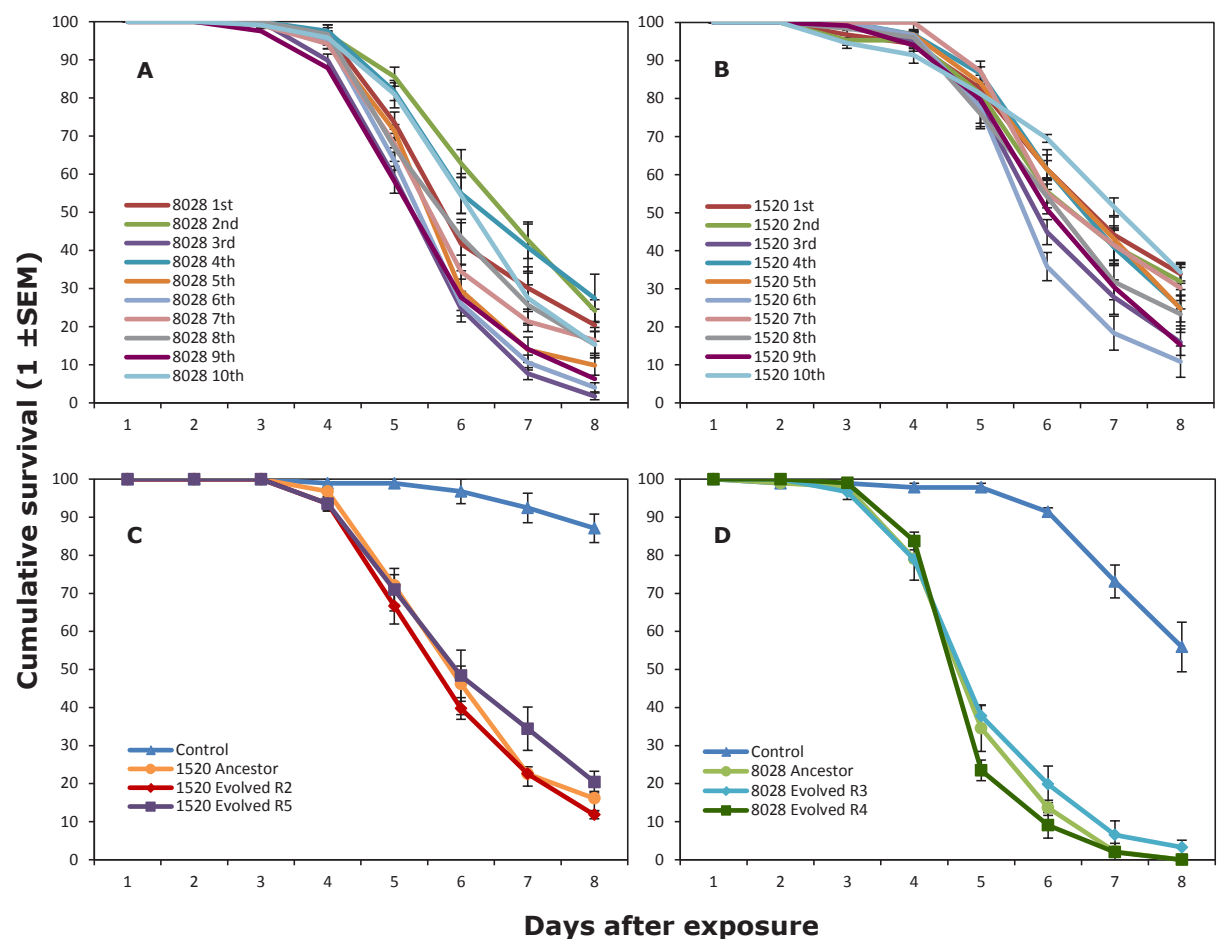

Figure 5. Daily proportional survival of Anopheles coluzzii infected with spores of different Beauveria bassiana isolates. In the control treatments, mosquitoes were exposed to only Shellsol $T^{\oplus}$ oil. During the course of the experimental evolution experiment, 10 selection cycles were completed and the daily mortality of mosquitoes was recorded for each cycle (A-B). Two lineages of the evolved isolates from Bb1520 (C) and Bb8028 (D) were tested against their respective ancestor. Data show means \pm SEM from five $(A-B)$ or three $(C-D)$ replicates of 40 female mosquitoes.

5 from isolate Bb1520 (Figure 5C) were not significantly different from their ancestor $\left(\mathrm{x}_{2}=2.59\right.$, d.f. $=2, p=0.275$ ). Also, replicates 3 and 4 of isolate Bb8028 (Figure 5D) were not significantly different from their ancestor $\left(\mathrm{x}_{2}=3.82\right.$, d.f. $\left.=2, \mathrm{p}=0.148\right)$.

\section{Quantification of fungal growth rate on dead mosquitoes}

Evolutionary lineage 4 from isolate Bb8028 was used again in a bioassay and the mortality rate was not significantly different from its ancestor $\left(\mathrm{x}_{2}=1.84\right.$, d.f. $\left.=1, \mathrm{p}=0.175\right)$. Dead mosquitoes from this experiment were sampled two, four and six days after their time of death. The fungal biomass (as measured as the number of copies of genomes) was not significantly different between the evolved and ancestor of isolate Bb8028 $\left(\mathrm{F}_{1,14}=0.173, \mathrm{p}=0.684\right)$. Nevertheless, the fungal biomass differed depending on the time sampled $\left(\mathrm{F}_{2,15}=102.8, \mathrm{p}<0.001\right)$, in which there was a lower quantity of fungal biomass at day two compared to day four and six (Figure S2). 


\section{Experimental evolution}

\section{Discussion}

Increased efficacy of entomopathogenic fungi is an important pre-requisite for their successful deployment as a biological control agent of insects. To this end, various approaches have been employed for investigating the underlying genetic and physiological mechanisms of fungal pathogenicity towards insects (reviewed in Valero-Jiménez et al. (2016)). In this study, we applied an experimental evolution approach in which $B$. bassiana was used to infect malaria mosquitoes for 10 consecutive selection cycles. We first assessed whether our selection scheme could result in the fixation of (general) genetic changes and, secondly, whether we could select for higher fungal virulence using this approach. We observed different mycelial growth rates and sporulation rates between evolved and ancestral isolates when growing the fungus on different growth media, confirming that evolutionary changes had occurred during our experimental evolution. The direction of change in growth rate and sporulation rate in the evolved lineages originating from Bb8028 (high lethality) depended on the growth media. On the contrary, the evolved lineages originating from Bb1520 (intermediate lethality) on average had no change or a decrease in growth rate and sporulation. Similarly, Scully \& Bidochka (2005), found that after five cycles through a host, there was a decreased ability of Aspergillus flavus to grow on an artificial nutrient source, suggesting that this could precede an adaptation to increase virulence on a given host. Remarkably, the evolved lineages of Bb8028 on average had increased growth and sporulation rates when grown on trehalose as a carbon source as compared to the ancestor. Trehalose is the principal sugar that circulates in the haemolymph of insects (Thompson, 2003) and the occurrence of high concentrations of trehalose in conidia of entomopathogenic fungi have been associated with increased virulence (Andersen et al., 2006). A knock-out mutant defective in a carbohydrate transporter gene (BbAGT1) displayed a decreased utilization of trehalose, germination, and decreased virulence in both topical and intrahaemocoel injection bioassays (Wang et al., 2013). An increased utilization of trehalose in all evolved Bb8028 lineages could indicate that adaptation to an insect host is taking place. When comparing trehalose results to the changes in growth rate and sporulation on insect cuticle medium, it can be inferred that the internal environment puts a stronger selection pressure on these fungi than the external (cuticle) environment.

In this study, we did not observe any significant change in virulence in 10 lineages originating from two isolates of $B$. bassiana after 10 selection cycles using malaria mosquitoes as a host. Previous studies reported that entomopathogenic fungi that have been serially passed through an insect host have acquired an increased virulence (Adames et al., 2011; Hayden et al., 1992; Quesada-Moraga and Vey, 2003; Song and Feng, 2011). For example, an isolate of $B$. bassiana increased in virulence after two cycles through Locusta migratoria (Quesada-Moraga 


\section{Chapter 5}

and Vey, 2003). However, similar studies have reported that no enhancement of virulence of entomopathogenic fungi after cycling through an insect hosts was observed (Latch, 1976; Scully and Bidochka, 2005; Vandenberg and Cantone, 2004). For instance, three strains of Paecilomyces fumosoroseus after 15 cycles through insects did not acquire an increased virulence and, in some cases, virulence was even reduced (Vandenberg and Cantone, 2004). These mixed results indicate that it is important to have a sufficient number of evolution cycles and that assessing phenotypic change includes comparing evolved lineages and the ancestor in one experimental assay. Indeed, also in the course of our experiments after a certain number of passages virulence seemed to have increased (Fig. 5A, B). Moreover, (subtle) variation in the species used and the experimental protocol may result in shifts in which traits and aspects of the biological interaction between fungus and insect are actually under selection. We will discuss this in more detail for our experiments below.

For our experimental evolution setup, we hypothesized that during selection cycles, beneficial mutations might arise that increase the fitness (virulence) and therefore be selectively enriched in the fungal population, especially since $B$. bassiana displays extensive natural variation in virulence against mosquitoes (Valero-Jiménez et al., 2014). Nevertheless, when starting an evolution experiment with a clonal population, there is a delay in the detection of fitness changes in the population due to the low-frequency emergence of beneficial mutations, random genetic drift, the magnitude of the selective advantage, and clonal interference (Long et al., 2015). We also considered that during this experimental setup, not only an increase in virulence would be advantageous to $B$. bassiana, but also an increased rate in sporulation once the host is dead. As spores from a dead mosquito were used to inoculate a new batch of mosquitoes, a mutation that would increase sporulation would be selected over time. Nevertheless, we could not detect any differences in rate of fungal outgrowth after the host had died.

In conclusion, our results show that prolonged selection of $B$. bassiana on malaria mosquitoes as the sole medium for growth can lead to evolutionary changes in fungal growth characteristics. These include changes in mycelial growth and sporulation rate, but not in fungal virulence. Infection of insects by $B$. bassiana is a complex trait that involves several steps including attachment of conidia to a host cuticle, germination of conidia, breaching of the cuticle, secretion of toxins, evasion of the immune system, and sporulation from the dead insect body (Valero-Jiménez et al., 2016). Due to the complexity of this trait and the intrinsic variation of the host insect, we suggest that a variation in the experimental setup is required to understand the components of fungal virulence and to be able to select for increased virulence. Nutrition is a key factor in immune function of insects (Lee et al., 2008), therefore it is unlikely that the immune response to fungal 


\section{Experimental evolution}

infection is constant over time. This is related to the general "quality" of the mosquitoes from our stock, which is known to vary between batches. This would significantly reduce the selection efficacy because mosquitoes would succumb from their poor fitness rather than because of high virulence of the fungal genotype with which it is infected. Thus, we suggest either using an insect for which quality is better controlled (e.g. Drosophila melanogaster) and/or that cycling through a dead insect host would give a better opportunity to detect significant differences of the evolved lineages using experimental evolution. Also, the use of a low-virulent isolate as a starting ancestor would provide an increased selective benefit of increased virulence, and therefore increasing the odds of detecting phenotypic changes in less selection cycles. To further study evolutionary changes using experimental evolution in B. bassiana, additional experiments should consider the results obtained in this study. Despite our results, the use of experimental evolution to naturally adapt to a particular host could still be a good tool to study host-pathogen interactions, and understand the genetic mechanisms involved in fungal virulence. The understanding of genetic mechanisms will improve our methods to use B. bassiana as a viable biological control agent against malaria mosquitoes. 


\section{Chapter 5}

\section{References}

Adames, M., Fernández-ruvalcaba, M., Peńa-chora, G., Vìctor, M.H.-V., 2011. Effects of passages through a suitable host of the fungus, Metarhizium anisopliae, on the virulence of acaricide- susceptible and resistant strains of the tick, Rhipicephalus microplus. J Insect Sci 11, 1-13.

Andersen, M., Magan, N., Mead, a., Chandler, D., 2006. Development of a population-based threshold model of conidial germination for analysing the effects of physiological manipulation on the stress tolerance and infectivity of insect pathogenic fungi. Environ Microbiol 8, 1625-1634. doi:10.1111/j.1462-2920.2006.01055.x

Bell, A.S., Blanford, S., Jenkins, N., Thomas, M.B., Read, A.F., 2009. Real-time quantitative PCR for analysis of candidate fungal biopesticides against malaria: technique validation and first applications. J Invertebr Pathol 100, 160-8. doi:10.1016/j.jip.2009.01.006

Blanford, S., Shi, W., Christian, R., Marden, J.H., Koekemoer, L.L., Brooke, B.D., Coetzee, M., Read, A.F., Thomas, M.B., 2011. Lethal and pre-lethal effects of a fungal biopesticide contribute to substantial and rapid control of malaria vectors. PLoS One 6, e23591. doi:10.1371/journal.pone.0023591

de Crecy, E., Jaronski, S., Lyons, B., Lyons, T.J., Keyhani, N.O., 2009. Directed evolution of a filamentous fungus for thermotolerance. BMC Biotechnol 9, 74. doi:10.1186/1472-6750-9-74

Fang, W., Leng, B., Xiao, Y., Jin, K., Ma, J., Fan, Y., Feng, J., Yang, X., Zhang, Y., Pei, Y., 2005. Cloning of Beauveria bassiana chitinase gene Bbchit1 and its application to improve fungal strain virulence. Appl Environ Microbiol 71, 363-370. doi:10.1128/AEM.71.1.363-370.2005

Farenhorst, M., Mouatcho, J.C., Kikankie, C.K., Brooke, B.D., Hunt, R.H., Thomas, M.B., Koekemoer, L.L., Knols, B.G.J., Coetzee, M., 2009. Fungal infection counters insecticide resistance in African malaria mosquitoes. Proc Natl Acad Sci U S A 106, 1744317447. doi:10.1073/pnas.0908530106

Hayden, T.P., Bidochka, M.J., Khachatourians, G.G., 1992. Entomopathogenicity of Several Fungi toward the English Grain Aphid (Homoptera, Aphididae) and Enhancement of Virulence with Host Passage of Paecilomyces farinosus. J Econ Entomol 85, 58-64.

Howard, A.F. V, Koenraadt, C.J.M., Farenhorst, M., Knols, B.G.J., Takken, W., 2010. Pyrethroid resistance in Anopheles gambiae leads to increased susceptibility to the entomopathogenic fungi Metarhizium anisopliae and Beauveria bassiana. Malar J 9, 168. doi:10.1186/14752875-9-168

Howard, A.F. V, N'Guessan, R., Koenraadt, C.J.M., Asidi, A., Farenhorst, M., Akogbéto, M., Knols, B.G.J.,
Takken, W., 2011. First report of the infection of insecticide-resistant malaria vector mosquitoes with an entomopathogenic fungus under field conditions. Malar J 10, 24. doi:10.1186/1475-2875-10-24

Jaronski, S.T., 2010. Ecological factors in the inundative use of fungal entomopathogens. BioControl 55, 159185. doi:10.1007/s10526-009-9248-3

Kamareddine, L., Fan, Y., Osta, M.A., Keyhani, N.O., 2013. Expression of trypsin modulating oostatic factor (TMOF) in an entomopathogenic fungus increases its virulence towards Anopheles gambiae and reduces fecundity in the target mosquito. Parasit Vectors 6, 22. doi:10.1186/1756-3305-6-22

Kanzok, S.M., Jacobs-Lorena, M., 2006. Entomopathogenic fungi as biological insecticides to control malaria. Trends Parasitol 22, 49-51. doi:10.1016/j.pt.2005.12.008

Knols, B.G.J., Bukhari, T., Farenhorst, M., 2010. Entomopathogenic fungi as the next-generation control agents against malaria mosquitoes. Future Microbiol 5 , 339-341. doi: $10.2217 / \mathrm{fmb} .10 .11$

Koenraadt, C.J.M., Takken, W., 2011. Viability of GM Fungi Crucial to Malaria Control. Science (80- ) 332, 175-175. doi:10.1126/science.332.6026.175

Latch, G.C.M., 1976. Studies on the susceptibility of Oryctes rhinoceros to some entomogenous fungi. Entomophaga 21, 31-38. doi:10.1007/BF02372013

Lee, K.P., Simpson, S.J., Wilson, K., 2008. Dietary protein-quality influences melanization and immune function in an insect. Funct Ecol 22, 1052-1061. doi:10.1111/j.1365-2435.2008.01459.x

Li, Z., Alves, S.B., Roberts, D.W., Fan, M., Delalibera, I., Tang, J., Lopes, R.B., Faria, M., Rangel, D.E.N., 2010. Biological control of insects in Brazil and China: history, current programs and reasons for their successes using entomopathogenic fungi. Biocontrol Sci Technol 20, 117-136. doi:10.1080/09583150903431665

Long, A., Liti, G., Luptak, A., Tenaillon, O., 2015. Elucidating the molecular architecture of adaptation via evolve and resequence experiments. Nat Publ Gr 16, 567-582. doi:10.1038/nrg3937

Lu, D., Pava-Ripoll, M., Li, Z., Wang, C., 2008. Insecticidal evaluation of Beauveria bassiana engineered to express a scorpion neurotoxin and a cuticle degrading protease. Appl Microbiol Biotechnol 81, 515-22. doi:10.1007/s00253-008-1695-8

Mnyone, L.L., Lyimo, I.N., Lwetoijera, D.W., Mpingwa, M.W., Nchimbi, N., Hancock, P.A., Russell, T.L., Kirby, M.J., Takken, W., Koenraadt, C.J.M., 2012. Exploiting the behaviour of wild malaria vectors to achieve high infection with fungal biocontrol agents. Malar J 11, 87. doi: $10.1186 / 1475-2875-11-87$ 


\section{Experimental evolution}

Quesada-Moraga, E., Vey, A., 2003. Intra-specific Variation in Virulence and In Vitro Production of Macromolecular Toxins Active Against Locust Among Beauveria bassiana Strains and Effects of In Vivo and In Vitro Passage on These Factors Intra-specific Var. Biocontrol Sci Technol 13, 323-340.

Ranson, H., Lissenden, 2016. Insecticide resistance in African Anopheles mosquitos: A worsening situation that needs urgent action to maintain malaria control. Trends Parasitol, 187-196. doi:10.1016/j.pt.2015.11.010

Rehner, S.A., Minnis, A.M., Sung, G.-H., Luangsa-ard, J.J., Devotto, L., Humber, R.A., 2011. Phylogeny and systematics of the anamorphic, entomopathogenic genus Beauveria. Mycologia 103, 1055-73. doi:10.3852/10302

Robledo-Monterrubio, M., Alatorre-Rosas, R., ViniegraGonzález, G., Loera, O., 2009. Selection of improved Beauveria bassiana (Bals.) Vuill. strains based on 2-deoxy-D-glucose resistance and physiological analysis. J Invertebr Pathol 101, 222-7. doi:10.1016/j. jip.2009.05.007

Scholte, E.-J., Knols, B.G.J., Takken, W., 2006. Infection of the malaria mosquito Anopheles gambiae with the entomopathogenic fungus Metarhizium anisopliae reduces blood feeding and fecundity. J Invertebr Pathol 91, 43-9. doi:10.1016/j.jip.2005.10.006

Schoustra, S.E., Punzalan, D., Dali, R., Rundle, H.D., Kassen, R., 2012. Multivariate Phenotypic Divergence Due to the Fixation of Beneficial Mutations in Experimentally Evolved Lineages of a Filamentous Fungus. PLoS One 7, 1-7. doi:10.1371/journal. pone.0050305

Scully, L.R., Bidochka, M.J., 2005. Serial passage of the opportunistic pathogen Aspergillus flavus through an insect host yields decreased saprobic capacity. Can J Microbiol 51, 185-9. doi:10.1139/w04-124

Shapiro-Ilan, D.I., Reilly, C.C., Hotchkiss, M.W., Wood, B.W., 2002. The potential for enhanced fungicide resistance in Beauveria bassiana through strain discovery and artificial selection. J Invertebr Pathol 81, 86-93. doi:http://dx.doi.org/10.1016/S0022-2011(02)00151-9

Song, T.T., Feng, M.G., 2011. In vivo passages of heterologous Beauveria bassiana isolates improve conidial surface properties and pathogenicity to Nilaparvata lugens (Homoptera: Delphacidae). J Invertebr Pathol 106, 211-216. doi:10.1016/j.jip.2010.09.022

Thomas, M.B., Read, A.F., 2007. Can fungal biopesticides control malaria? Nat Rev Microbiol 5, 377-383. doi:10.1038/nrmicro1638

Thompson, S.N., 2003. Trehalose - The Insect "Blood" Sugar, in: Advances in Insect Physiology. pp. 205-285. doi:10.1016/S0065-2806(03)31004-5
Valero-Jiménez, C.A., Debets, A.J.M., van Kan, J.A.L., Schoustra, S.E., Takken, W., Zwaan, B.J., Koenraadt, C.J.M., 2014. Natural variation in virulence of the entomopathogenic fungus Beauveria bassiana against malaria mosquitoes. Malar J 13, 479. doi:10.1186/14752875-13-479

Valero-Jiménez, C.A., Wiegers, H., Zwaan, B.J., Koenraadt, C.J.M., van Kan, J.A.L., 2016. Genes involved in virulence of the entomopathogenic fungus Beauveria bassiana. J Invertebr Pathol 133, 41-49. doi:10.1016/j.jip.2015.11.011

Vandenberg, J.D., Cantone, F. a, 2004. Effect of serial transfer of three strains of Paecilomyces fumosoroseus on growth in vitro, virulence, and host specificity. J Invertebr Pathol 85, 40-5. doi:10.1016/j.jip.2003.12.004

Viaud, M., Couteaudier, Y., Riba, G., 1998. Molecular analysis of hypervirulent somatic hybrids of the entomopathogenic fungi Beauveria bassiana and Beauveria sulfurescens. Appl Environ Microbiol 64, 8893.

Wang, X.-X., Ji, X.-P., Li, J.-X., Keyhani, N.O., Feng, M.-G., Ying, S.-H., 2013. A putative $\alpha$-glucoside transporter gene BbAGT1 contributes to carbohydrate utilization, growth, conidiation and virulence of filamentous entomopathogenic fungus Beauveria bassiana. Res Microbiol 164, 480-9. doi:10.1016/j. resmic.2013.02.008

Xie, X.-Q., Wang, J., Huang, B.-F., Ying, S.-H., Feng, M.-G., 2010. A new manganese superoxide dismutase identified from Beauveria bassiana enhances virulence and stress tolerance when overexpressed in the fungal pathogen. Appl Microbiol Biotechnol 86, 1543-53. doi:10.1007/s00253-010-2437-2 


\section{Chapter 5}

\section{Supplementary materials}

\section{Data S1}

Fungal growth was measured when grown on 35 different media differing in the carbon source, in order to further investigate differences between ancestral and evolved lineages of Bb1520 and Bb8028. The fungi were grown on a minimal medium containing: $0.5 \mathrm{~g} \mathrm{MgSO} \cdot 7 \mathrm{H}_{2} \mathrm{O}, 1.5 \mathrm{~g}$ L-aspargine (monohydrate), $1 \mathrm{ml}$ of Solution A, $1 \mathrm{ml}$ of Solution B, $1 \mathrm{ml}$ of Solution C, $2.5 \mathrm{ml}$ of Solution D, $5 \mathrm{ml}$ of urea $(1 \mathrm{M})$, and select agar $15 \mathrm{~g}$ in 11 of distilled water. Solution A consisted of thiaminiumdichloride $(0.012 \mathrm{~g} / 100 \mathrm{ml})$; Solution $\mathrm{B}$ : $\mathrm{FeCl}_{3} \cdot 6 \mathrm{H}_{2} \mathrm{O}(0.5 \mathrm{~g} / 100 \mathrm{ml})$; Solution C: $\mathrm{HBO}_{3}(0.06 \mathrm{~g} / \mathrm{l}),\left(\mathrm{NH}_{4}\right) \mathrm{Mo}_{7} \mathrm{O}_{24} \cdot 4 \mathrm{H}_{2} \mathrm{O}(0.04 \mathrm{~g} / \mathrm{l}), \mathrm{CuSO}_{4} \cdot 5 \mathrm{H}_{2} \mathrm{O}(0.2 \mathrm{~g} / \mathrm{l}), \mathrm{ZnSO}_{4} \cdot 7 \mathrm{H}_{2} \mathrm{O}(2.0$ $\mathrm{g} / \mathrm{l}), \mathrm{MnSO}_{4} \cdot 4 \mathrm{H}_{2} \mathrm{O}(0.1 \mathrm{~g} / \mathrm{l}), \mathrm{CoCl}_{2} \cdot 6 \mathrm{H}_{2} \mathrm{O}(0.4 \mathrm{~g} / \mathrm{l})$, and $\mathrm{Ca}\left(\mathrm{NO}_{3}\right)_{2} \cdot 4 \mathrm{H}_{2} \mathrm{O}(1.2 \mathrm{~g} / \mathrm{l})$; Solution D: $\mathrm{KH}_{2} \mathrm{PO}_{4}(184 \mathrm{~g} / \mathrm{l})$, and $\mathrm{K}_{2} \mathrm{HPO}_{4} \cdot 3 \mathrm{H}_{2} \mathrm{O}(524.05 \mathrm{~g} / \mathrm{l})$.

The minimum media was complemented with one source of carbon, that consisted of: $30 \mathrm{~g}$ alfalfa meal (AM), $10 \mathrm{~g}$ apple pectin (Ap), $10 \mathrm{~g}$ arabic gum (A gum), $10 \mathrm{~g}$ arabinogalactan (arab g), $10 \mathrm{~g}$ beechwood xylan (BX), $10 \mathrm{~g}$ casein (Cas), $8.5 \mathrm{~g}$ cellobiose (CB), $10 \mathrm{~g}$ cellulose (Cel), $10 \mathrm{~g}$ chitin $(\mathrm{CH}), 10 \mathrm{~g}$ citrus pectin $(\mathrm{Cp}), 30 \mathrm{~g}$ citrus pulp (cit), $30 \mathrm{~g}$ cotton seed pulp (CSP), 4.5 g D-fructose (Fru), 4.5 g D-galactose (G), 5.4 g D-galacturonic acid (gala A), $5 \mathrm{~g}$ D-glucose (Glu), 4.85 g D-glucuronic acid (glu A), 4.5 g D-mannose (Man), 3.75 g D-ribose (Rib), 3.75 g D-xylose (Xyl), 10 g guar gum (G gum), 10 g inulin (Inu), 9 g lactose (Lac), 3.75 $\mathrm{g}$ L-arabinose (L-ara), $10 \mathrm{~g}$ lignin hydrolytic (Lig), $4.5 \mathrm{~g}$ L-rhamnose (RH), $9 \mathrm{~g}$ maltose (MT), $14.85 \mathrm{~g}$ raffinose $(\mathrm{R}), 30 \mathrm{~g}$ rice bran (rice), $10 \mathrm{~g}$ soluble starch (SS), $30 \mathrm{~g}$ soybean hulls (Soy), 8.5 $\mathrm{g}$ sucrose (Suc), $30 \mathrm{~g}$ sugar beet pulp (SBP), $30 \mathrm{~g}$ and wheat bran (Wb). 

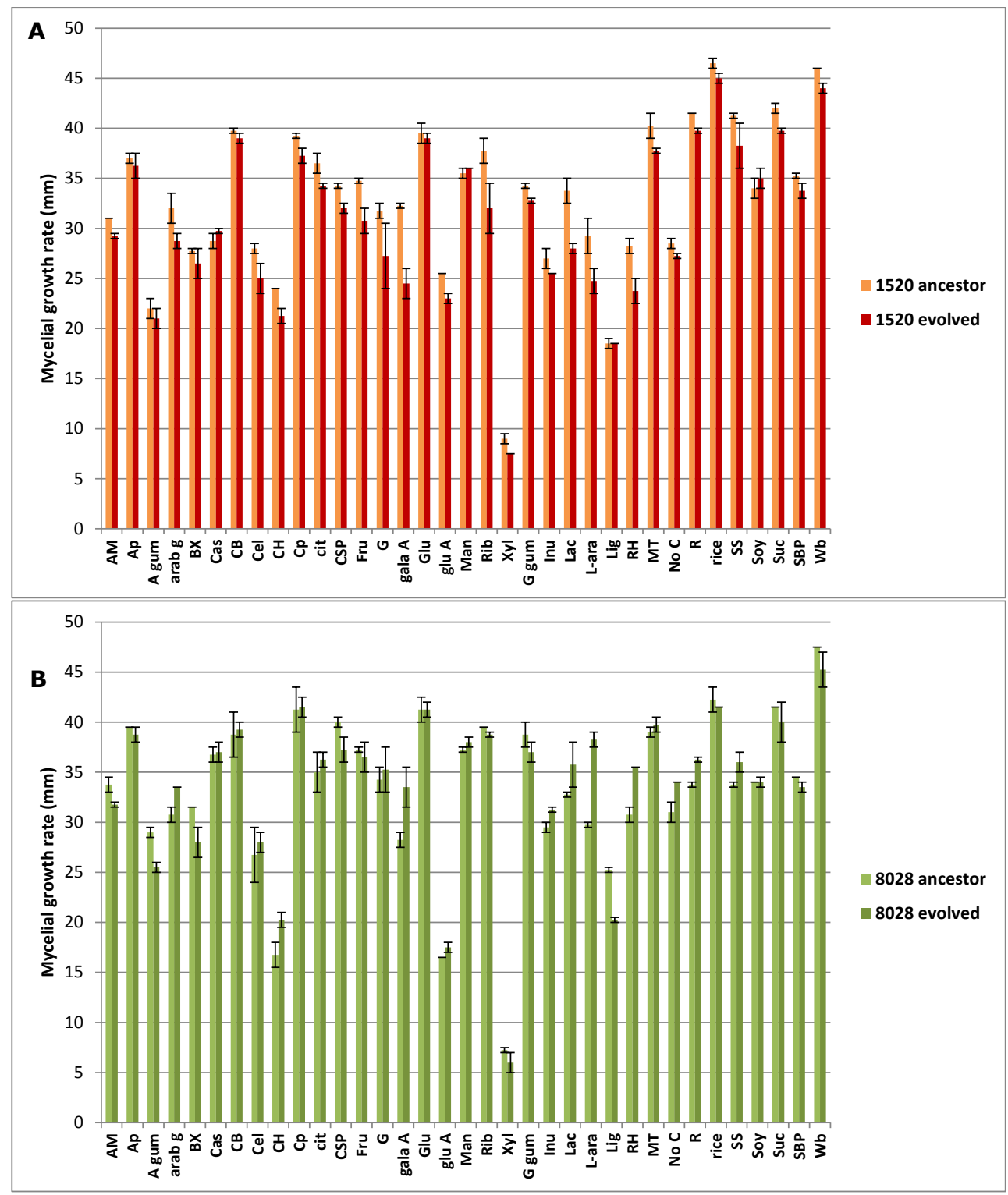

Figure S1. Bar chart representing the mycelial growth rate of Beauveria bassiana grown on 35 different media that differ in the carbon source: alfalfa meal (AM), apple pectin (Ap), arabic gum (A gum), arabinogalactan (arab g), beechwood xylan (BX), casein (Cas), cellobiose (CB), cellulose $(\mathrm{Cel})$, chitin $(\mathrm{CH})$, citrus pectin $(\mathrm{Cp})$, citrus pulp (cit), cotton seed pulp (CSP), D-fructose (Fru), D-galactose (G), D-galacturonic acid (gala A), D-glucose (Glu), D-glucuronic acid (glu A), D-mannose (Man), D-ribose (Rib), D-xylose (Xyl), guar gum (G gum), inulin (Inu), lactose (Lac), L-arabinose (L-ara), lignin hydrolytic (Lig), L-rhamnose (RH), maltose (MT), no carbon source (No C), raffinose (R), rice bran (rice), soluble starch (SS), soybean hulls (Soy), sucrose (Suc), sugar beet pulp (SBP), and wheat bran (Wb). Panel A shows the comparison of the ancestor and evolved lineage of Bb1520, while panel B shows the comparison of the ancestor and evolved lineage 4 of Bb8028. Data shown present the means \pm SEM from two replicates. 


\section{Chapter 5}

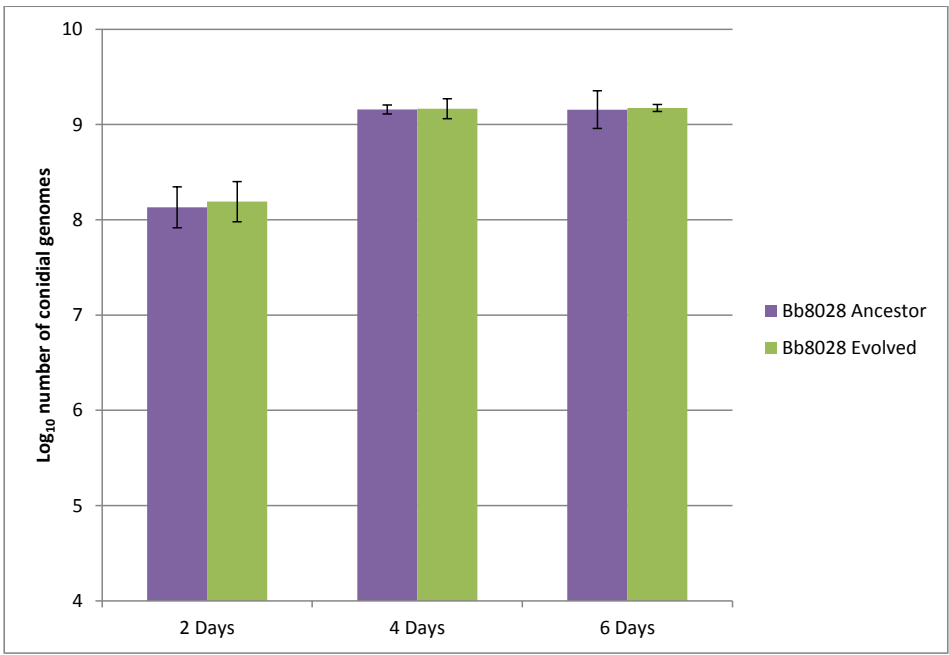

Figure S2. Bar chart representing the number of Beauveria bassiana conidial units on/within infected mosquitoes with respect to time after death. Lineage four of isolate Bb8028 from the 10th cycle and spores of Bb8028 from the initial bioassay were used. Data shown present the means \pm SEM from three replicates. 


\title{
Chapter 6
}

\author{
Discussion
}




\section{Chapter 6}

\section{Malaria is still a major killer}

Malaria is a major threat to human health in large parts of the world and reducing this burden is much needed. In 2000, it was estimated that the number of deaths due to malaria was 839,000 resulting from around 262 million malaria cases. By 2015, the number of estimated deaths had decreased to 438,000 ( $48 \%$ reduction) and the number of malaria cases to 214 million (18\% reduction) (World Health Organization, 2015). Most of this reduction can be attributed to malaria control interventions such as insecticide-treated bed nets (ITNs), indoor residual spraying (IRS), and artemisinin-based combination therapy (ACT). It has been estimated that these malaria control interventions have averted 663 million clinical cases since 2000, of which $68 \%, 22 \%$, and $10 \%$ were attributed to ITNs, ACTs, and IRS, respectively (Bhatt et al., 2015).

The efforts and progress made over the last decade to control and eradicate malaria are now threatened by the emergence of insecticide resistance in Anopheles mosquitoes and artemisinin resistance in Plasmodium falciparum. Insecticide resistance in malaria mosquitoes is present throughout Africa, and recent studies have shown that for the only class of insecticides allowed for use in ITNs (pyrethroids) the effectiveness is decreasing over time (Ranson and Lissenden, 2016). For instance, mosquitoes from a locality in Uganda exposed to $0.05 \%$ deltamethrin in a standardized World Health Organization (WHO) susceptibility bioassay in 2006 showed almost 100\% mortality. Similar studies in the same locality in 2013 showed that mortality by deltamethrin was around $40 \%$, which indicates the development and rapid spread of insecticide resistance (Okia et al., 2013; Verhaeghen et al., 2010). Moreover, artemisinin resistance in Plasmodium falciparum has been reported in Southeast Asia, specifically in Myanmar, Cambodia, Thailand, Vietnam, and Laos (Ashley et al., 2014). Although artemisinin resistance has not yet reached Africa, a study revealed that Anopheles species present in Africa are able to be competent vectors of artemisinin-resistant $P$. falciparum (St. Laurent et al., 2015), which would pose a serious challenge to global efforts to eliminate malaria. Because of the negative implications of insecticide resistance and artemisinin resistance on malaria control, the development of alternative tools for malaria vector control is urgently needed.

\section{Entomopathogenic fungi as a biocontrol alternative}

In this thesis, the use of entomopathogenic fungi (EPF) as a biological control agent of malaria mosquitoes has been investigated. Previous research on EPF and malaria mosquitoes has shown that EPF are an effective tool to reduce malaria transmission under laboratory and field conditions (Blanford et al., 2005; Mnyone et al., 2012; Scholte et al., 2005). However, fundamental knowledge on the mechanisms and regulation of the infection process in the fungus, as well as insights into the defensive responses to EPF in the host insect is limited. The majority of 


\section{Discussion}

studies has focused on global regulatory genes that control the expression of many downstream genes, but not on specific virulence factors. Although these studies have led to useful insights, most of the findings are confirmatory (i.e. the function is already known from other fungi) or fragmented. Therefore, to assess whether EPF may provide a robust and sustainable solution to kill malaria mosquitoes, a better understanding is needed of the life history of the biological control agent as well as the mechanisms and modes of action in killing the host. Such knowledge can help improve biocontrol agents, not only by selecting the most virulent genotypes, but also by selecting the genotypes that use combinations of virulence mechanisms for which resistance in the insect host is least likely to develop. With this in mind, this thesis focussed on understanding the genetic mechanisms of Beauveria bassiana virulence towards malaria mosquitoes using a multidisciplinary approach (Figure 1). First, the current knowledge about genes related to virulence in B. bassiana was reviewed (Chapter 2). The natural variation in virulence among a collection of 29 isolates of $B$. bassiana toward malaria mosquitoes was investigated (Chapter 3), and from this collection, I selected five isolates of contrasting virulence for genomic studies. A comparative genomics analyses was performed in order to obtain insights in the mechanisms explaining these contrasts in virulence (Chapter 4). Furthermore, an experimental evolution approach was used in an attempt to improve fungal virulence towards malaria mosquitoes (Chapter 5).

\section{Contributions to a better EPF implementation}

The main findings of this thesis are, (1) that there is extensive natural variation in virulence of $B$. bassiana isolates against malaria mosquitoes (up to 10-fold between the least and most virulent isolate), which helps to determine the choices for selecting the optimal fungal isolates for controlling malaria vectors. (2) This variation appeared to be unrelated to phenotypic characteristics of the fungal isolates, such as spore size, mycelial growth rate, sporulation, or geographical origin. (3) By using a comparative genomics approach on five isolates representing the extremes of low and high virulence, I have generated a high quality near-complete genome assembly for the most virulent isolate (Bb8028), and identified isolate-specific genes that might have a role in virulence. These genes are interesting candidates for future functional analyses by, for instance, making targeted knock-out mutants. (4) Furthermore, using experimental evolution, which is an effective tool to identify genes and mechanisms underpinning natural variation in virulence, I observed that prolonged selection of $B$. bassiana on malaria mosquitoes as the sole medium for growth for the fungus led to evolutionary changes in fungal growth characteristics, but not in virulence. In the current chapter, I will further integrate the results found in this thesis and address how they are related to each other. I will address several aspects and challenges that need to be considered in order to implement EPF on a large scale as a 


\section{Chapter 6}

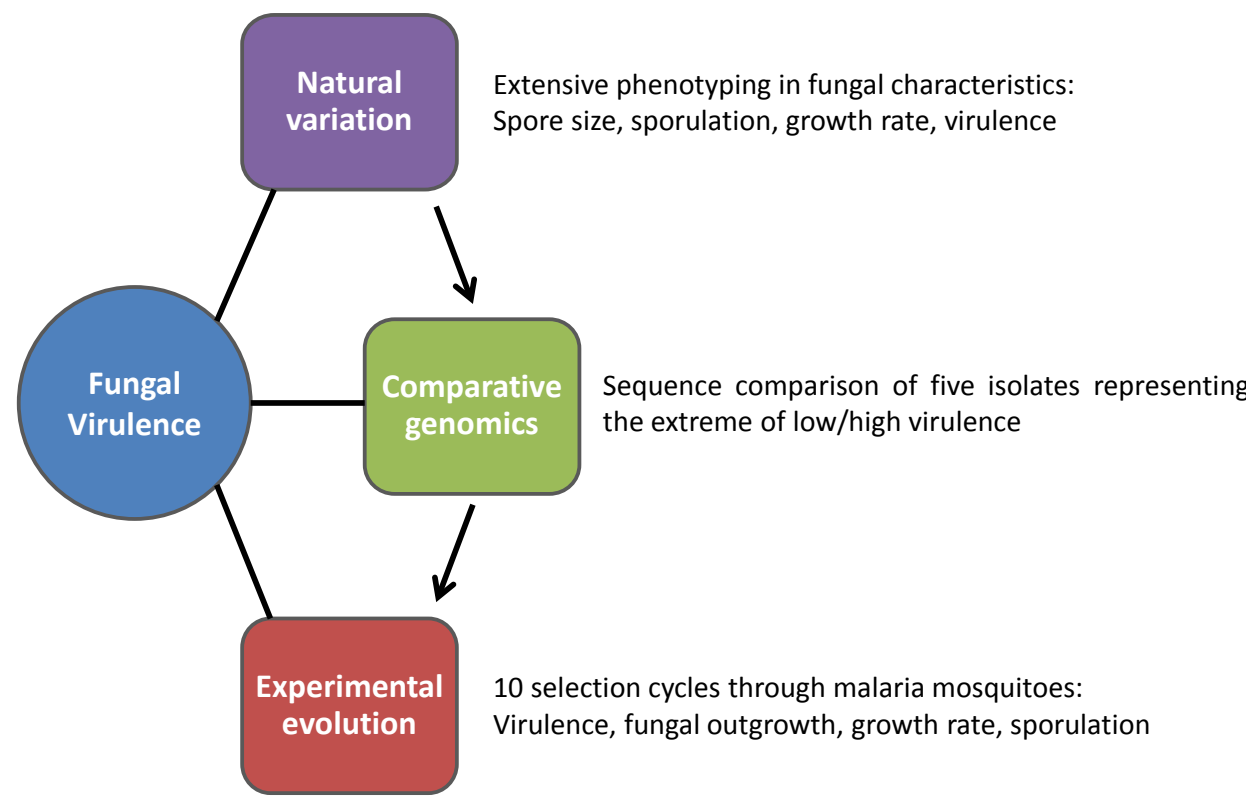

Figure 1. Overview of the multidisciplinary approach used in my thesis to study fungal virulence in $B$. bassiana against malaria mosquitoes. First, I investigated the natural variation in virulence among a collection of 29 isolates of $B$. bassiana toward malaria mosquitoes as well as several fungal phenotypic characteristics. Secondly, from this collection I selected five isolates of contrasting virulence for whole genomic studies. I performed a comparative genomics analysis in order to obtain insights in the mechanisms explaining these contrasts in virulence. Thirdly, I used an experimental evolution approach in an attempt to improve fungal virulence towards malaria mosquitoes using two isolates that differed in virulence and that had their genome sequenced.

biological control agent of malaria mosquitoes. Finally, I will suggest future research lines that will deepen our understanding in the key factors involved in virulence of B. bassiana, which consequently will improve our methods to use this fungus as an effective biological control agent against vectors of human disease.

\section{Factors affecting transmission to the insect}

Currently, one of the major challenges that affects the use of entomopathogenic fungi in field applications is their relatively poor and unpredictable performance compared to traditional insecticides. Ideally, optimal EPF would be "evolution-proof", i.e. with a low likelihood that mosquitoes can develop resistance to EPF. In addition, EPF should be highly virulent, easily mass-produced, with low production cost, with a long-term storage, and high persistence in the environment. At present the viability, infectivity, and persistence of EPF under field conditions are inadequate. This is partly caused by variations in environmental factors such as temperature, humidity, and UV exposure that affect the fungal viability, as well as persistence and virulence (Jaronski, 2010). For instance, fungal spores stored at $4^{\circ} \mathrm{C}$ and then placed at different 


\section{Discussion}

temperatures showed a clear temperature-dependent decline in viability, with decay half-lives of 31,49 and 71 days for $32^{\circ} \mathrm{C}, 26^{\circ} \mathrm{C}$ and $22^{\circ} \mathrm{C}$, respectively (Blanford et al., 2012). The tolerance of conidia of $44 \mathrm{~B}$. bassiana isolates to UV-B exposure varied widely among isolates, with LD50s from 0.15 to $0.93 \mathrm{~J} \mathrm{~cm}^{-2}$. However, all the LD50 estimates were substantially lower than a cumulative UV-B dose of $2.4 \mathrm{~J} \mathrm{~cm}^{-2}$ measured from sunlight on a sunny day in summer (Yao et al., 2010). Humidity is also a factor that affects spore persistence, and studies have shown that conidia are able to survive longer at lower values $(<50 \%)$ of relative humidity (Sandhu et al., 1993). Despite these hurdles, there are approaches that focus on improving efficacy of EPF based on physiological manipulation of the fungus during production, or on the development of better formulation and application strategies. For instance, an emulsion of EPF conidia in vegetable oil protects the conidia from UV light-induced damage and increases their efficacy and virulence by promoting conidial adhesion on the insect's cuticle surfaces (Luz et al., 2012; Mishra et al., 2013).

Another challenge for the implementation of EPF is that in contrast to chemical insecticides, EPF do not cause quick mortality in mosquitoes but rather kill slowly via a complex process. This process starts with the attachment of spores to the insect cuticle, penetration of the cuticle, and then proliferation within the haemocoel, which ultimately causes death. The slow speed of killing insects by EPF has been identified as a disadvantage for their practical application (Lacey et al., 2015; Ward, 2005). However, slow killing still makes EPF effective for malaria control since only old mosquitoes are able to transmit malaria (Knols et al., 2010). Once a female mosquito is infected with Plasmodium, the parasite needs around 10-14 days to mature, and only after a new blood meal, they are able to infect a new human host (Killeen et al., 2000). Therefore, if EPF are able to infect mosquitoes early in life, they would have sufficient time to kill older mosquitoes before they are able to transmit the parasite. Even if mosquitoes are exposed to EPF later in life, Blanford et al. (2011) reported that these mosquitoes suffered from a substantial reduction in feeding propensity, with full cessation of taking blood meals after four days of inoculation with the fungus. Moreover, while EPF-infected mosquitoes were still able to lays egg before dying, they did so to a lesser extent.

\section{More with less}

In Chapter 3, I focused on the natural variation among B. bassiana isolates in virulence against mosquitoes because, among other things, virulence determines the choices for selecting the optimal fungal isolates for controlling malaria vectors. Chapter 3 describes that the most virulent isolate can display an increased effectiveness of up to ten-fold compared to the least virulent isolate. It should, however, be considered that using a more virulent isolate will not 


\section{Chapter 6}

necessarily increase its effectiveness on reducing malaria transmission rate when evaluated under conditions simulating a realistic field situation (Valero-Jiménez et al., unpublished data). It has been shown that there is a dose-dependent effect of spore concentration (Blanford et al., 2011; Farenhorst and Knols, 2010), so a highly virulent isolate could have a similar effect using a lower concentration compared to a less virulent isolate. Reducing the amount of inoculum required to achieve satisfactory control is crucial since there is a physical and economical limit to the amount of spores that can be produced and applied (Lacey et al., 2015).

\section{The value of comparative genomics}

This thesis provides important insights into virulence of the entomopathogenic fungus B. bassiana towards malaria mosquitoes. The study of natural variation in fungal virulence has revealed the extent of variation in the different components of virulence and their overall role. Based on the ample natural variation observed in fungal virulence, a comparative genomics analysis (Chapter 4) has brought us a step closer to understanding the mechanisms of fungal virulence, which warrants future work to unravel the biological complexity of entomopathogenic fungi in general, and $B$. bassiana in particular. In other fungal pathogens, the use of comparative genomics has gained great insights in the virulence mechanisms and host interactions. For instance, in the plant pathogen Fusarium oxysporum, comparative genomics led to the discovery of four extra chromosomes that are enriched for transposable elements and genes putatively related to hostpathogen interactions (Perez-Nadales et al., 2014). Furthermore, there is experimental evidence that these chromosomes can be transferred horizontally, as shown between two strains of $F$. oxysporum that resulted in a transfer of small chromosomes from a tomato pathogenic to a nonpathogenic strain, converting the latter into a pathogen (Ma et al., 2010). Another example can be found in Ustilago maydis, a fungal pathogen of maize, in which the use of comparative genomics allowed detection of so-called "divergence clusters". Many of these gene clusters encode effectors that have undergone specialization during evolution (Schirawski et al., 2010). In the comparative genomics study of B. bassiana I found 390 genes that are Bb8028 isolate-specific, from which a subset could have putative roles in virulence. Nevertheless, I could not find clear patterns that could explain the contrasting variation observed in virulence in the five sequenced isolates. This could be related to the complex strategy that B. bassiana uses to infect insects. Contrasting to some plant pathogenic fungi, in which virulence can be determined by a few effector genes (e.g. small secreted cysteine-rich proteins), current knowledge of $B$. bassiana infection cycle in insects (Chapter 2) indicates that it is a complex trait that involves several steps including attachment of conidia to a host cuticle, germination of conidia, breaching of the cuticle, secretion of toxins, evasion of the immune system, and sporulation from the dead insect body. Moreover, many of the genes in these processes are potentially redundant, and nonspecific in nature they can 


\section{Discussion}

contribute to other processes such as saprophytic growth, conidiation, and stress tolerance.

Furthermore, important insights in understanding the host-pathogen interactions during the fungal infection could also be gained by analysing gene expression. For instance, analysing the transcriptomes of both host and pathogen can provide new insights into this process by identifying new virulence factors in B. bassiana, or new pathways in the mosquitoes that respond specifically to fungi (Westermann et al., 2012). Detailed monitoring of the genes expressed from both the fungi and the mosquito, can be done from the start of the infection process, (e.g. when a spore adheres to the mosquito cuticle), throughout the infection (e.g. when spores breach the cuticle, and grow as blastospores), and until the fungus prevails and kills the mosquito. This will enable a temporal characterization of responses and changes in gene expression in both organisms. Additionally, fungal isolates with contrasting virulence have different growth dynamics, which in combination with the regulation of expression of genes will help elucidate the mechanisms of host-pathogen interactions during the fungal infection. For instance, following the growth dynamics of isolate Bb8028 (highly virulent) showed that the fungal burden is reduced between days 1 and 3 post-exposure compared to "pick-up densities" at exposure (Figure 2). Then, the burden increases from day 4 until day 6 , which coincides with the time that no mosquitoes remain alive. In contrast, the growth dynamics of isolate $\mathrm{Bb} 5078$ (slightly virulent) is very different; after a reduction in fungal burden in days 1-4, there is only a slight increase in fungal burden during days 5-9, followed by a small decrease in day 10 and 11, after which all mosquitoes have died. Insights from these studies can also be used to screen for isolates expressing higher levels of candidate genes as markers for more virulent isolates.

Once putative virulence factors are identified (e.g., via comparative genomics or gene expression analysis), a detailed functional analysis of the molecular mechanisms has to be done. The most common approach utilized to elucidate the function of these genes involves reverse genetics, where the functional gene is replaced by an antibiotic selection marker (Selin et al., 2016). For B. bassiana, an Agrobacterium-mediated transformation method was developed using a phosphinothricin acetyltransferase (bar) gene as a selectable marker (Fang et al., 2004). Also, RNAi-mediated gene expression suppression has been successfully used in B. bassiana in identifying the function of two manganese-dependent superoxide dismutases (Xie et al., 2012). A challenge arises when targeting genes with redundant functions within large protein families such as chitinases. However, novel methods such as CRISPR-Cas9 have been developed for filamentous fungi that provide a simple and versatile way to introduce mutations in fungi, that holds promises to be useful to target multiple genes and help dissect the function of many virulence factors in EPF (Nødvig et al., 2015). 


\section{Chapter 6}

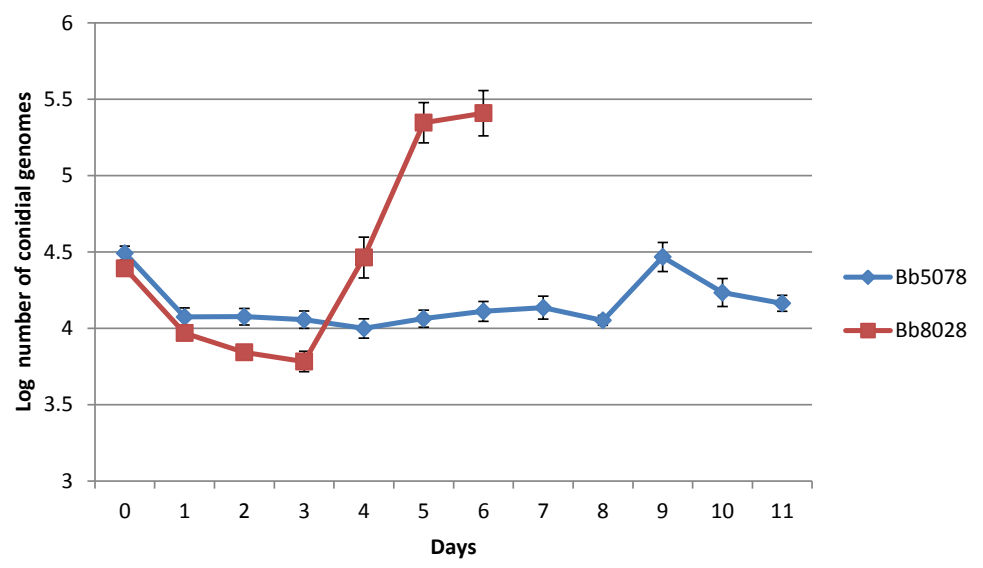

Figure 2. $\log _{10}$ number of Beauveria bassiana conidia on/within mosquitoes with respect to time post-challenge. Error bars are standard errors of the mean across 15 replicates.

\section{Experimental evolution, a method with potential}

Another approach that I took to study and improve virulence of $B$. bassiana as a biological control agent against malaria mosquitoes was experimental evolution (EE; Chapter 5). The main goal was to find out whether EE could result in evolved fungal strains with higher virulence against mosquitoes. B. bassiana has a wide range of host insects and can grow as an endophyte inside plants and in soil as a saprophyte. By forcing the fungus to use malaria mosquitoes as the sole nutritional resource for 10 consecutive selection cycles, I expected the fungus to develop as a specialist towards mosquitoes. To use a specialist would be safer because a narrower host range might avoid non-target effects on the ecosystem (Zhao et al., 2016). My results show that prolonged selection of $B$. bassiana on malaria mosquitoes as the sole medium for growth can lead to evolutionary changes in fungal growth characteristics (mycelial growth and sporulation rate), but not in virulence towards malaria mosquitoes. The lack of an increase of virulence was unanticipated and hampered my aim to uncover the genes and genetic pathways underpinning (natural) virulence. The original conceptual strategy was to use an "evolve and resequence" approach that would allow us to analyse the genetic changes that had occurred in the evolved isolates (Long et al., 2015) as a complement to the comparative genomics approach (Chapter 4) to identify genes and mechanisms underpinning natural variation in virulence.

Despite these results, I believe the use of experimental evolution (EE) to naturally adapt to a particular host could still be a good tool to study host-pathogen interactions and understand the 


\section{Discussion}

genetic mechanisms involved in fungal virulence. One of the challenges in doing experimental evolution is to maintain a high and constant quality of mosquitoes as substrate for fungal growth and development over time. Fitness of mosquitoes can be influenced by population density, food availability, and climatic conditions, which can strongly influence the outcome of experiments (Spitzen and Takken, 2005). During the course of EE, I observed variations in mosquito fitness, which could have significantly reduced the selection efficacy because mosquitoes would succumb from "poor fitness" rather than because of high virulence of the fungal genotype with which it is infected. Thus, I suggest either using an insect for which quality is better controlled (e.g. Drosophila melanogaster) and/or that cycling through a dead insect host would give a better opportunity to detect significant differences of the evolved lineages using experimental evolution. Furthermore, conducting EE for a significant longer time could potentially still lead to observable changes in fungal virulence. This is because when starting an evolution experiment with a clonal population there is a delay in the detection of fitness changes in the population due to the low-frequency emergence of beneficial mutations, random genetic drift, the magnitude of the selective advantage, and clonal interference (Long et al., 2015). In the 10 consecutive selection cycles in mosquitoes, I estimated that only around 80-150 fungal mitotic generations occurred in total. Therefore, more generations of EE will allow for uncovering the molecular determinants of adaptation to mosquitoes. Contrasting to the high level of genetic diversity found in B. bassiana (Chapter 4), using EE would allow me to better identify the genetic changes correlated with beneficial alleles that increase virulence between the evolved and ancestral genotypes. Thus, using EE represents an essential resource for understanding the genetic mechanisms involved in fungal virulence.

\section{The risk of resistance}

Unquestionably, mosquitoes are capable to adapt and develop resistance to almost all classes of insecticides used thus far (Ranson and Lissenden, 2016). Therefore, it is expected that this could also occur regarding resistance to EPF. Despite B. bassiana being able to infect a broad range of arthropods, there are reports of insects that are refractory to infection by $B$. bassiana. For instance, the red flour beetle Tribolium castaneum is not susceptible to infection by B. bassiana, and this is attributed to its ability to produce quinone-containing cuticular secretions that act as antimicrobial defence compounds (Pedrini et al., 2015; Yezerski et al., 2007). Nevertheless, as explained above, EPF have a lower killing capacity than insecticides, which allows mosquitoes to reproduce before dying. Consequently, EPF will not impose a high selective pressure since a beneficial mutation (i.e. resistance towards fungal infection) would confer fitness benefits after most individuals have ceased reproducing. Thus, the selection pressure for developing resistance against fungal infections is lower (Read et al., 2009). Also, in contrast to other pathogens, EPF 


\section{Chapter 6}

overcome insect defences by secretion of many enzymes and toxic molecules, fast assimilation of nutrients, inhibition of defence responses, and rapid growth (Ortiz-Urquiza and Keyhani, 2016). All this decreases the probability of mosquitoes developing resistance towards such an array of toxic components and physical challenges. Even if mosquitoes could develop resistance to EPF, most likely such resistance mechanisms would be metabolically costly and all the individuals in a population would pay the price for a benefit that is experienced only by a few (Thomas and Read, 2007).

Another aspect to consider is that in order for mosquitoes to become infected with EPF, they require physical contact to a surface (e.g. walls, fabrics, nets) that has been impregnated with fungal spores. Mosquitoes would gain a selective advantage if they are able to detect and avoid the fungal spores or able to display post-contact responses such as grooming to reduce the risk of infection (Baverstock et al., 2010). This behavioural response would considerably reduce the efficiency of EPF as a biological control agent. The avoidance of fungi by insects has been observed in species within Coleoptera, Isoptera, Hemiptera and Orthoptera. For instance, the termites Macrotermes michaelseni were able to detect a virulent isolate of Metarhizium anisopliae from a distance and avoided direct physical contact. Furthermore, avoidance was positively correlated with the virulence of the isolate used (Mburu et al., 2009). Similarly, the mole cricket Scapteriscus spp. when exposed to spores of B. bassiana displayed changes in behaviour that reduced exposure to the conidia, such as less surface tunnelling, less tunnelling along the perimeter of the containers and remaining in areas with less spores (Thompson et al., 2005). Although, recent studies have shown that malaria mosquitoes do not show any avoidance or repellence behaviour against EPF (George et al., 2013; Mnyone et al., 2010), it could be a possibility given enough time. A solution to avoid this issue would be to use different isolates or EPF that have different levels of attractiveness/repellence, combined with chemical agents that reduce the repellence factor of the EPF, or use it in combination with attractive blends (Okumu et al., 2010). Another approach is to employ EPF using strategies that would take advantage of the behaviour of malaria mosquitoes. For instance, mosquitoes are attracted to dark colours (Browne and Bennett, 1981), so spraying EPF on black surfaces could increase exposure. EPF can also be used in combination with a bed-net, which would exploit the natural attractiveness of mosquitoes to the host sleeping under the net (Mnyone et al., 2012).

Additionally, genetic diversity of natural populations of mosquitoes has to be taken into consideration. Some genotypes could be more susceptible to fungal infection than others, which could lead to an increased possibility of resistance (Mitri and Vernick, 2012). To address this point of differential effects of $B$. bassiana for different insect genotypes, I conducted an experiment using 


\section{Discussion}

(A) EE: Early reproduction/Female

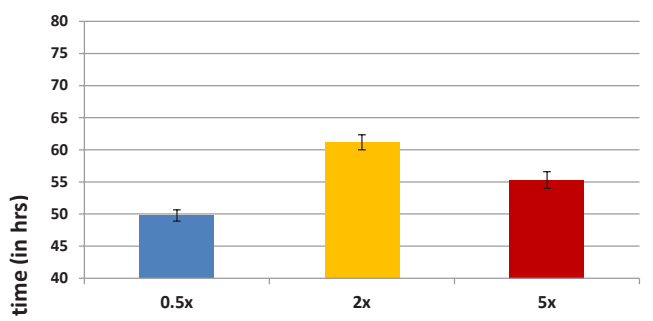

(C) EE: Early reproduction/Male

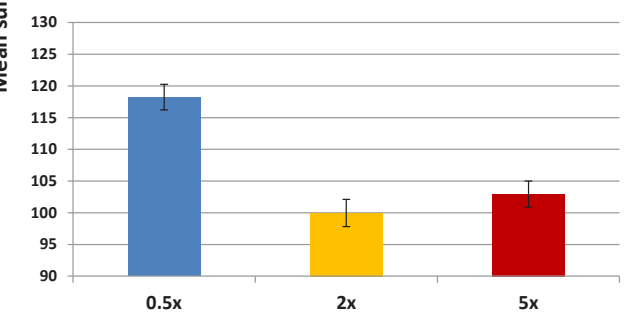

(B) EE: Late reproduction/Female

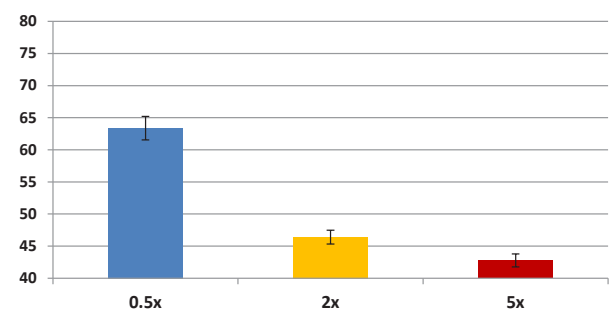

(D) EE: Late reproduction/Male

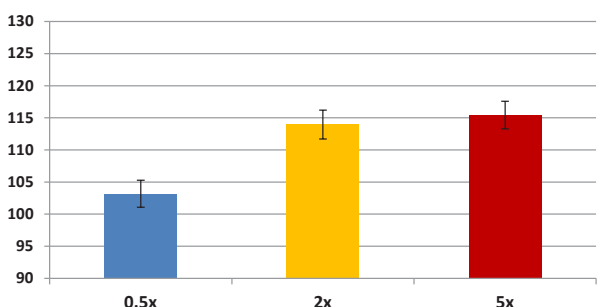

Figure 3. Mean survival time of Drosophila melanogaster experimentally evolved lines against infection of Beauveria bassiana. Blue, yellow and red represent the poor $(0.5 \mathrm{X})$, control (2X) and rich (5X) EE larval diets respectively. Lines from each larval diet reproduced 14 days post egg-laying (Early, A, C) and at 28 days post egg laying (Late, B, D). Error bars are standard errors of the mean across four replicate lines.

24 fruit fly lines (Drosophila melanogaster), which were previously evolved using experimental evolution. The EE fruit fly lines were adapted to three different diets (poor (0.5X), control (2X), rich $(5 \mathrm{X})$ ) during development in combination with selection for early or late reproduction during adulthood (May et al., in prep). The results clearly showed that susceptibility to fungal infection depends on the sex of the flies, food type conditions, and age-at-reproduction (Figure 3). Male fruit flies raised in control and rich food selected for late reproduction (Figure 3C) survived longer than flies raised in control and rich food selected for early reproduction (Figure 3D), while female flies selected for early reproduction raised in control and rich food (Figure $3 \mathrm{~A})$ survived longer than flies raised in control and rich food selected for late reproduction (Figure 3B). The opposite trend was observed for flies raised in control food. These results suggest that there is genetic variation for susceptibility to fungal infection, and that different developmental environments and adult demography can determine the level of susceptibility. Therefore, EPF may show variation in effectiveness in different geographic areas and ecological conditions. A means to break any resistance in the insect would be to use different isolates or EPF that have different virulence mechanisms, which exploits the evolutionary potential of the pathogen. Nevertheless, in experiments done by Blanford et al. (2011), in which they infected eight colonies of Anopheles gambiae s.s. with B. bassiana, no significant difference was found in 


\section{Chapter 6}

median survival time between colonies. In light of these contrasting results, further experiments should test several wild populations of An. gambiae with B. bassiana.

\section{From the lab to the field}

I believe that a better understanding in the fundamental aspects of the biology and ecology of $\mathrm{EPF}$ and malaria mosquitoes in the natural environment is of great value in the development of more sustainable approaches to control malaria vectors. I also believe that in order to successfully implement EPF as a biological control agent, we need to integrate EPF into our current set of vector control tools such as ITNs and IRS that are already in use. To facilitate this, an integrated vector management (IVM) approach has to be implemented, which consists of a rational decision-making process that takes into consideration the efficacy, cost effectiveness, ecological soundness and sustainability of vector control interventions and optimizes the use of resources for vector control (Who, 2012). Combining the use of EPF with current insecticides could provide a valuable addition since EPF have different modes of action, which could reduce even more the risks of developing resistance of mosquitoes against EPF. Besides using EPF with current control tools, other methods also have to be considered such as modification of the environment (Imbahale et al., 2012; Kirby et al., 2008), genetic modification of mosquitoes (Lofgren et al., 1974), odour baiting traps systems (Menger et al., 2014), and larval control with other biological control agents (Fillinger and Lindsay, 2006; Kusumawathie et al., 2008). By using several methods in an IVM system approach, long-term sustainability of malaria vector control can be achieved. As previous research on EPF and malaria mosquitoes has shown that EPF are an effective tool to kill malaria mosquitoes and reduce malaria transmission under laboratory and semi-field conditions (Blanford et al., 2005; Mnyone et al., 2012; Scholte et al., 2005; this thesis), future efforts should focus on large-scale field trials to demonstrate the effectiveness of EPF not only on killing mosquitoes but on their potential to reduce malaria transmission. 


\section{Discussion}

\section{References}

Ashley, E. a., Dhorda, M., Fairhurst, R.M., Amaratunga, C., Lim, P., Suon, S., Sreng, S., Anderson, J.M., Mao, S., Sam, B., Sopha, C., Chuor, C.M., Nguon, C., Sovannaroth, S., Pukrittayakamee, S., Jittamala, P., Chotivanich, K., Chutasmit, K., Suchatsoonthorn, C., Runcharoen, R., Hien, T.T., Thuy-Nhien, N.T., Thanh, N.V., Phu, N.H., Htut, Y., Han, K.-T., Aye, K.H., Mokuolu, O. a., Olaosebikan, R.R., Folaranmi, O.O., Mayxay, M., Khanthavong, M., Hongvanthong, B., Newton, P.N., Onyamboko, M. a., Fanello, C.I., Tshefu, A.K., Mishra, N., Valecha, N., Phyo, A.P., Nosten, F., Yi, P., Tripura, R., Borrmann, S., Bashraheil, M., Peshu, J., Faiz, M.A., Ghose, A., Hossain, M.A., Samad, R., Rahman, M.R., Hasan, M.M., Islam, A., Miotto, O., Amato, R., MacInnis, B., Stalker, J., Kwiatkowski, D.P., Bozdech, Z., Jeeyapant, A., Cheah, P.Y., Sakulthaew, T., Chalk, J., Intharabut, B., Silamut, K., Lee, S.J., Vihokhern, B., Kunasol, C., Imwong, M., Tarning, J., Taylor, W.J., Yeung, S., Woodrow, C.J., Flegg, J. a., Das, D., Smith, J., Venkatesan, M., Plowe, C. V., Stepniewska, K., Guerin, P.J., Dondorp, A.M., Day, N.P., White, N.J., 2014. Spread of Artemisinin Resistance in Plasmodium falciparum Malaria. N Engl J Med 371, 411-423. doi:10.1056/NEJMoa1314981

Baverstock, J., Roy, H.E., Pell, J.K., 2010. Entomopathogenic fungi and insect behaviour: From unsuspecting hosts to targeted vectors. BioControl 55, 89-102. doi:10.1007/s10526-009-9238-5

Bhatt, S., Weiss, D.J., Cameron, E., Bisanzio, D., Mappin, B., Dalrymple, U., Battle, K.E., Moyes, C.L., Henry, A., Eckhoff, P.A., Wenger, E.A., Briët, O., Penny, M.A., Smith, T.A., Bennett, A., Yukich, J., Eisele, T.P., Griffin, J.T., Fergus, C.A., Lynch, M., Lindgren, F., Cohen, J.M., Murray, C.L.J., Smith, D.L., Hay, S.I., Cibulskis, R.E., Gething, P.W., 2015. The effect of malaria control on Plasmodium falciparum in Africa between 2000 and 2015. Nature 526, 207-211. doi:10.1038/nature15535

Blanford, S., Chan, B.H.K., Jenkins, N., Sim, D., Turner, R.J., Read, A.F., Thomas, M.B., 2005. Fungal pathogen reduces potential for malaria transmission. Science 308, 1638-41. doi:10.1126/science.1108423

Blanford, S., Jenkins, N.E., Christian, R., Chan, B.H.K., Nardini, L., Osae, M., Koekemoer, L., Coetzee, M., Read, A.F., Thomas, M.B., 2012. Storage and persistence of a candidate fungal biopesticide for use against adult malaria vectors. Malar J 11, 354. doi:10.1186/14752875-11-354

Blanford, S., Shi, W., Christian, R., Marden, J.H., Koekemoer, L.L., Brooke, B.D., Coetzee, M., Read, A.F., Thomas, M.B., 2011. Lethal and pre-lethal effects of a fungal biopesticide contribute to substantial and rapid control of malaria vectors. PLoS One 6, e23591. doi:10.1371/journal.pone.0023591
Browne, S.M., Bennett, G.F., 1981. Response of mosquitoes (Diptera: Culicidae) to visual stimuli. J Med Entomol. 18, 505-521.

Fang, W., Zhang, Y., Yang, X., Zheng, X., Duan, H., Li, Y., Pei, Y., 2004. Agrobacterium tumefaciens-mediated transformation of Beauveria bassiana using an herbicide resistance gene as a selection marker. J Invertebr Pathol 85, 18-24. doi:10.1016/j.jip.2003.12.003

Farenhorst, M., Knols, B.G.J., 2010. A novel method for standardized application of fungal spore coatings for mosquito exposure bioassays. Malar J 9, 27. doi:10.1186/1475-2875-9-27

Fillinger, U., Lindsay, S.W., 2006. Suppression of exposure to malaria vectors by an order of magnitude using microbial larvicides in rural Kenya. Trop Med Int Heal 11, 1629-1642. doi:10.1111/j.1365-3156.2006.01733.x

George, J., Jenkins, N.E., Blanford, S., Thomas, M.B., Baker, T.C., 2013. Malaria mosquitoes attracted by fatal fungus. PLoS One 8, e62632. doi:10.1371/journal. pone. 0062632

Imbahale, S.S., Githeko, A., Mukabana, W.R., Takken, W., 2012. Integrated mosquito larval source management reduces larval numbers in two highland villages in western Kenya. BMC Public Health 12, 362. doi:10.1186/14712458-12-362

Jaronski, S.T., 2010. Ecological factors in the inundative use of fungal entomopathogens. BioControl 55, 159185. doi:10.1007/s10526-009-9248-3

Killeen, G.F., McKenzie, F.E., Foy, B.D., Schieffelin, C., Billingsley, P.F., Beier, J.C., 2000. A simplified model for predicting malaria entomologic inoculation rates based on entomologic and parasitologic parameters relevant to control. Am J Trop Med Hyg 62, 535-44.

Kirby, M.J., West, P.A., Green, C., Jasseh, M., Lindsay, S.W., 2008. Risk factors for house-entry by culicine mosquitoes in a rural town and satellite villages in The Gambia. Parasites and Vectors 1, 41. doi:10.1186/17563305-1-41

Knols, B.G.J., Bukhari, T., Farenhorst, M., 2010. Entomopathogenic fungi as the next-generation control agents against malaria mosquitoes. Future Microbiol 5, 339-341. doi:10.2217/fmb.10.11

Kusumawathie, P.H., Wickremasinghe, A.R., Karunaweera, N.D., Wijeyaratne, M.J., 2008. Larvivorous potential of the guppy, Poecilia reticulata, in anopheline mosquito control in riverbed pools below the Kotmale dam, Sri Lanka. Asia Pac J Public Heal 20, 56-63. doi:10.1177/1010539507308507

Lacey, L.A., Grzywacz, D., Shapiro-Ilan, D.I., Frutos, R. Brownbridge, M., Goettel, M.S., 2015. Insect pathogens as biological control agents: Back to the future. J Invertebr Pathol 132, 1-41. doi:10.1016/j.jip.2015.07.009 


\section{Chapter 6}

Lofgren, C.S., Dame, D.A., Breeland, S.G., Weidhaas, D.E., Jeffery, G., Kaiser, R., Ford, H.R., Boston, M.D., Baldwin, K.F., 1974. Release of chemosterilized males for the control of Anopheles albimanus in El Salvador. III. Field methods and population control. Am J Trop Med Hyg 23, 288-297.

Long, A., Liti, G., Luptak, A., Tenaillon, O., 2015. Elucidating the molecular architecture of adaptation via evolve and resequence experiments. Nat Publ Gr 16, 567-582. doi:10.1038/nrg3937

Luz, C., Rodrigues, J., Rocha, L.F.N., 2012. Diatomaceous earth and oil enhance effectiveness of Metarhizium anisopliae against Triatoma infestans. Acta Trop 122, 2935. doi:10.1016/j.actatropica.2011.11.014

Ma, L.-J., van der Does, H.C., Borkovich, K. a, Coleman, J.J., Daboussi, M.-J., Di Pietro, A., Dufresne, M., Freitag, M., Grabherr, M., Henrissat, B., Houterman, P.M., Kang, S., Shim, W.-B., Woloshuk, C., Xie, X., Xu, J.-R., Antoniw, J., Baker, S.E., Bluhm, B.H., Breakspear, A., Brown, D.W., Butchko, R. a E., Chapman, S., Coulson, R., Coutinho, P.M., Danchin, E.G.J., Diener, A., Gale, L.R., Gardiner, D.M., Goff, S., Hammond-Kosack, K.E., Hilburn, K., Hua-Van, A., Jonkers, W., Kazan, K., Kodira, C.D., Koehrsen, M., Kumar, L., Lee, Y.-H., Li, L., Manners, J.M., Miranda-Saavedra, D., Mukherjee, M., Park, G., Park, J., Park, S.-Y., Proctor, R.H., Regev, A., Ruiz-Roldan, M.C., Sain, D., Sakthikumar, S., Sykes, S., Schwartz, D.C., Turgeon, B.G., Wapinski, I., Yoder, O., Young, S., Zeng, Q., Zhou, S., Galagan, J., Cuomo, C. A., Kistler, H.C., Rep, M., 2010. Comparative genomics reveals mobile pathogenicity chromosomes in Fusarium. Nature 464, 367-73. doi:10.1038/nature08850

Mburu, D.M., Ochola, L., Maniania, N.K., Njagi, P.G.N., Gitonga, L.M., Ndung'u, M.W., Wanjoya, A.K., Hassanali, A., 2009. Relationship between virulence and repellency of entomopathogenic isolates of Metarhizium anisopliae and Beauveria bassiana to the termite Macrotermes michaelseni. J Insect Physiol 55, 774-780. doi:10.1016/j.jinsphys.2009.04.015

Menger, D.J., Otieno, B., de Rijk, M., Mukabana, W.R., van Loon, J.J., Takken, W., 2014. A push-pull system to reduce house entry of malaria mosquitoes. Malar J 13, 119. doi:10.1186/1475-2875-13-119

Mishra, S., Kumar, P., Malik, A., 2013. Preparation, characterization, and insecticidal activity evaluation of three different formulations of Beauveria bassiana against Musca domestica. Parasitol Res 112, 3485-3495. doi:10.1007/s00436-013-3529-6

Mitri, C., Vernick, K.D., 2012. Anopheles gambiae pathogen susceptibility: the intersection of genetics, immunity and ecology. Curr Opin Microbiol 15, 28591. doi:10.1016/j.mib.2012.04.001

Mnyone, L.L., Koenraadt, C.J., Lyimo, I.N., Mpingwa,
M.W., Takken, W., Russell, T.L., 2010. Anopheline and culicine mosquitoes are not repelled by surfaces treated with the entomopathogenic fungi Metarhizium anisopliae and Beauveria bassiana. Parasit Vectors 3, 80. doi:10.1186/1756-3305-3-80

Mnyone, L.L., Lyimo, I.N., Lwetoijera, D.W., Mpingwa, M.W., Nchimbi, N., Hancock, P.A., Russell, T.L., Kirby, M.J., Takken, W., Koenraadt, C.J.M., 2012. Exploiting the behaviour of wild malaria vectors to achieve high infection with fungal biocontrol agents. Malar J 11, 87. doi:10.1186/1475-2875-11-87

Nødvig, C.S., Nielsen, J.B., Kogle, M.E., Mortensen, U.H., 2015. A CRISPR-Cas9 System for Genetic Engineering of Filamentous Fungi. PLoS One 10, e0133085. doi:10.1371/journal.pone.0133085

Okia, M., Ndyomugyenyi, R., Kirunda, J., Byaruhanga, A., Adibaku, S., Lwamafa, D.K., Kironde, F., 2013. Bioefficacy of long-lasting insecticidal nets against pyrethroid-resistant populations of Anopheles gambiae s.s. from different malaria transmission zones in Uganda. Parasit Vectors 6, 130. doi:10.1186/1756-3305-6-130

Okumu, F.O., Killeen, G.F., Ogoma, S., Biswaro, L., Smallegange, R.C., Mbeyela, E., Titus, E., Munk, C., Ngonyani, H., Takken, W., Mshinda, H., Mukabana, W.R., Moore, S.J., 2010. Development and field evaluation of a synthetic mosquito lure that is more attractive than humans. PLoS One 5. doi:10.1371/ journal.pone.0008951

Ortiz-Urquiza, A., Keyhani, N.O., 2016. Molecular Genetics of Beauveria bassiana Infection of Insects, Advances in Genetics. Elsevier Ltd. doi:10.1016/ bs.adgen.2015.11.003

Pedrini, N., Ortiz-Urquiza, A., Huarte-Bonnet, C., Fan, Y., Juárez, M.P., Keyhani, N.O., 2015. Tenebrionid secretions and a fungal benzoquinone oxidoreductase form competing components of an arms race between a host and pathogen. Proc Natl Acad Sci 201504552. doi: $10.1073 /$ pnas. 1504552112

Perez-Nadales, E., Almeida Nogueira, M.F., Baldin, C., Castanheira, S., El Ghalid, M., Grund, E., Lengeler, K., Marchegiani, E., Mehrotra, P.V., Moretti, M., Naik, V., Oses-Ruiz, M., Oskarsson, T., Schäfer, K., Wasserstrom, L., Brakhage, A. A., Gow, N. A. R., Kahmann, R., Lebrun, M.H., Perez-Martin, J., Di Pietro, A., Talbot, N.J., Toquin, V., Walther, A., Wendland, J., 2014. Fungal model systems and the elucidation of pathogenicity determinants. Fungal Genet Biol 70, 4267. doi:10.1016/j.fgb.2014.06.011

Ranson, H., Lissenden, N., 2016. Insecticide Resistance in African Anopheles Mosquitoes: A Worsening Situation that Needs Urgent Action to Maintain Malaria Control. Trends Parasitol 32, 187-196. doi:10.1016/j. pt.2015.11.010 


\section{Discussion}

Read, A.F., Lynch, P. a, Thomas, M.B., 2009. How to make evolution-proof insecticides for malaria control. PLoS Biol 7, e1000058. doi:10.1371/journal.pbio.1000058

Sandhu, S.S., Rajak, R.C., Agarwal, G.P., 1993. Studies on Prolonged Storage of Beauveria bassiana Conidia: Effects of Temperature and Relative Humidity on Conidial Viability and Virulence against Chickpea Borer, Helicoverpa armigera. Biocontrol Sci Technol 3, 47-53. doi:10.1080/09583159309355258

Schirawski, J., Mannhaupt, G., Munch, K., Brefort, T., Schipper, K., Doehlemann, G., Di Stasio, M., Rossel, N., Mendoza-Mendoza, A., Pester, D., Muller, O., Winterberg, B., Meyer, E., Ghareeb, H., Wollenberg, T., Munsterkotter, M., Wong, P., Walter, M., Stukenbrock, E., Guldener, U., Kahmann, R., 2010. Pathogenicity Determinants in Smut Fungi Revealed by Genome Comparison. Science (80- ) 330, 1546-1548. doi:10.1126/science.1195330

Scholte, E.-J., Ng'habi, K., Kihonda, J., Takken, W., Paaijmans, K., Abdulla, S., Killeen, G.F., Knols, B.G.J., 2005. An entomopathogenic fungus for control of adult African malaria mosquitoes. Science (80- ) 308, 1641-2. doi: $10.1126 /$ science. 1108639

Selin, C., de Kievit, T.R., Belmonte, M.F., Fernando, W.G.D., 2016. Elucidating the Role of Effectors in PlantFungal Interactions: Progress and Challenges. Front Microbiol 7, 1-21. doi:10.3389/fmicb.2016.00600

Spitzen, J., Takken, W., 2005. Malaria mosquito rearing - maintaining quality and quantity of laboratory-reared insects. Proc Netherlands Entomol Meet 16, 95-100.

St. Laurent, B., Miller, B., Burton, T.A., Amaratunga, C., Men, S., Sovannaroth, S., Fay, M.P., Miotto, O., Gwadz, R.W., Anderson, J.M., Fairhurst, R.M., 2015. Artemisinin-resistant Plasmodium falciparum clinical isolates can infect diverse mosquito vectors of Southeast Asia and Africa. Nat Commun 6, 8614. doi:10.1038/ ncomms 9614

Thomas, M.B., Read, A.F., 2007. Can fungal biopesticides control malaria? Nat Rev Microbiol 5, 377-383. doi:10.1038/nrmicro1638

Thompson, S.R., Brandenburg, R.L., Brandenburg, R.L., 2005. Tunneling Responses of Mole Crickets (Orthoptera : Gryllotalpidae) to the Entomopathogenic Fungus, Beauveria bassiana 34, 140-147.

Verhaeghen, K., Van Bortel, W., Roelants, P., Okello, P.E., Talisuna, A., Coosemans, M., 2010. Spatiotemporal patterns in $k d r$ frequency in permethrin and DDT resistant Anopheles gambiae s.s. from Uganda. Am J Trop Med Hyg 82, 566-573. doi:10.4269/ ajtmh.2010.08-0668

Ward, M.D.W., 2005. Benefits and Risks in Malaria Control. Science (80- ) 310, 49c-51c. doi:10.1126/ science. $310.5745 .49 \mathrm{c}$

Westermann, A.J., Gorski, S. a, Vogel, J., 2012. Dual RNA-seq of pathogen and host. Nat Rev Microbiol 10, 618-30. doi:10.1038/nrmicro2852

World Health Organization, 2012. Handbook for integrated vector management. Outlooks Pest Manag 24, 1-78. doi:10.1564/v24_jun_14

World Health Organization, 2015. World Malaria Report 2014 Summary.

Xie, X.-Q., Li, F., Ying, S.-H., Feng, M.-G., 2012. Additive Contributions of Two Manganese-Cored Superoxide Dismutases (MnSODs) to Antioxidation, UV Tolerance and Virulence of Beauveria bassiana. PLoS One 7 , e30298. doi:10.1371/journal.pone.0030298

Yao, S.-L., Ying, S.-H., Feng, M.-G., Hatting, J.L., 2010 In vitro and in vivo responses of fungal biocontrol agents to gradient doses of UV-B and UV-A irradiation. BioControl 55, 413-422. doi:10.1007/s10526-0099265-2

Yezerski, A., Ciccone, C., Rozitski, J., Volingavage, B., 2007. The Effects of a Naturally Produced Benzoquinone on Microbes Common to Flour. J Chem Ecol 33, 1217 1225. doi:10.1007/s10886-007-9293-2

Zhao, H., Lovett, B., Fang, W., 2016. Genetically Engineering Entomopathogenic Fungi, Advances in Genetics. Elsevier Ltd. doi:http://dx.doi.org/10.1016/ bs.adgen.2015.11.001 



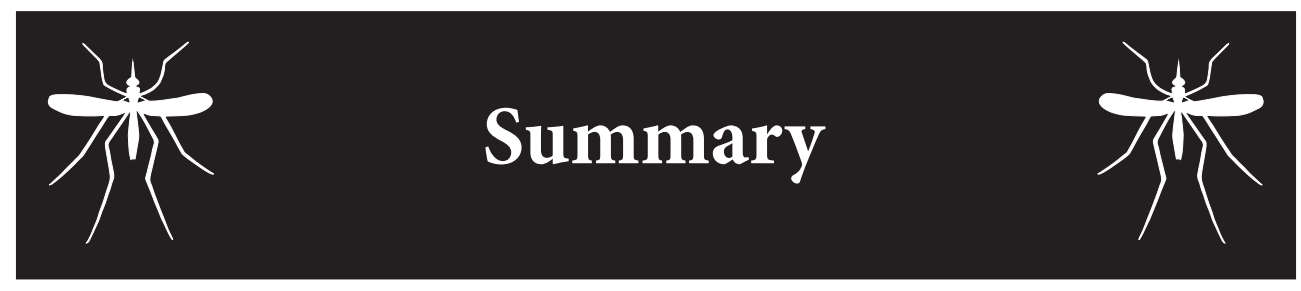


Although globally malaria mortality rates have fallen by $48 \%$ between 2000 and 2015, malaria is still killing an estimated 438,000 people each year. An effective way to alleviate the burden of malaria is to control its vector (malaria mosquitoes) using insecticides. This can be achieved either with insecticide-treated bed nets (ITNs) or through indoor residual spraying of insecticides (IRS). However, because of rapidly expanding insecticide resistance, there is a need to find alternatives to control the mosquitoes. Entomopathogenic fungi (EPF) could constitute an effective biological control tool, as is able to reduce malaria transmission under laboratory and field conditions. However, fundamental knowledge on the mechanisms and regulation of the infection process of the fungus, as well as insights into the defensive responses of the host insect to EPF, is limited. Therefore, to assess whether EPF may provide a robust and sustainable solution to control malaria mosquitoes, a better understanding is needed of the life history of the biological control agent as well as the mechanisms and modes of action in killing the host. Such knowledge can help improve biocontrol agents, not only by selecting the most virulent genotypes, but also by selecting genotypes that use combinations of virulence mechanisms for which resistance in the insect host is least likely to develop. Therefore, the aim of this thesis was to study virulence of the entomopathogenic fungus $B$. bassiana towards malaria mosquitoes using a multidisciplinary approach.

Chapter 2 provides an overview of existing knowledge of genes influencing virulence in EPF, with a special focus on $B$. bassiana. We divide the infection process into distinct steps: (1) host adherence, (2) germination and penetration peg development, (3) enzymatic cuticle degradation, (4) transition to blastospore growth, (5) colonization and killing of the host, (6) interactions with the insect immune system, and (7) hyphal extrusion and conidiation. We discuss each step in more detail and describe what genes and mechanisms influence virulence in B. bassiana. We also discuss the need for novel strategies and experimental methods to better understand the infection mechanisms deployed by entomopathogenic fungi.

The study of natural variation in fungal virulence is a first step towards understanding the genetic mechanisms involved, because it reveals the extent of variation in the different components of virulence, and has an impact on the choices for selecting the optimal fungal agents for controlling malaria vectors. Chapter 3 describes the natural variation in virulence for 29 B. bassiana isolates, originating from different parts of the world and representing multiple phylogenetic clades within the species, which were tested on malaria mosquitoes. In addition, phenotypic characteristics of the fungal isolates related to the infection steps described in Chapter 2, such as sporulation, spore size and growth rate were studied to explore their relationship with virulence. We found that all tested isolates of B. bassiana killed An. coluzzii mosquitoes, and the rate at 
which this happened differed significantly among the isolates. The risk of mosquitoes dying was around ten times higher when they were exposed to the most virulent as compared to the least virulent isolate. There was significant variation among isolates in spore size (1.95-2.75 $\mu \mathrm{m})$, growth rate $\left(1.77-2.71 \mathrm{~mm} /\right.$ day) and sporulation (from $3.5 \times 10^{5}-3.2 \times 10^{7}$ spores $/ \mathrm{cm}^{2}$ ), but none of these morphological characteristics were correlated, and thus predictive, for the ability of the fungal isolate to kill malaria mosquitoes. This study shows that there is a wide natural variation in virulence of isolates of $B$. bassiana, and that selecting an appropriate fungal isolate is highly relevant in killing and thus controlling malaria mosquitoes, especially if used as part of an integrated vector management strategy.

Based on the 10-fold natural variation observed in fungal virulence, Chapter $\mathbf{4}$ presents a comparative genomic analysis of five isolates representing the extremes of low and high virulence with the aim to uncover the genetic mechanisms underpinning virulence. A high-quality, nearcomplete genome assembly was achieved for the highly virulent isolate Bb8028 and it was compared to draft assemblies of four other isolates. Whole genome analysis showed a high level of genetic diversity between five isolates (2.85-16.81 SNPs/kb), which grouped into two distinct phylogenetic clusters. Mating type gene analysis revealed the presence of either the MAT 1-11 or the MAT 1-2-1 gene, and a putative new MAT gene was detected in the MAT 1-2 locus. Comparative genome analysis revealed that the most virulent isolate, Bb8028, contains 0.71 $\mathrm{Mb}$ (2.03\% of total genome) of unique sequences. Among the 390 unique genes of Bb8028 are a putative Non-Ribosomal Peptide Synthetase (NRPS) secondary metabolite gene cluster, and several genes with homology to bacterial toxin proteins. The Bb8028 genome assembly represents a significant resource for future research into the biology of this entomopathogenic fungus and its relevance as a biological control agent. Understanding the key factors involved in virulence of $B$. bassiana will improve our methods to use this fungus as an effective biological control agent against vectors of human disease.

The main goal of Chapter 5 was to find out whether experimental evolution (EE) could result in evolved fungal strains with higher virulence against mosquitoes. We quantified the virulence, fungal outgrowth (i.e. time of emergence from a dead host), mycelial growth rate (MGR), and sporulation rate of two B. bassiana isolates (Bb1520 and Bb8028) that underwent 10 consecutive selection cycles through malaria mosquitoes (Anopheles coluzzii). This cycling resulted in an altered capacity of evolved $B$. bassiana lineages to grow on different substrates while maintaining the ability to kill insects. Notably, however, there were no significant changes in virulence or speed of outgrowth when comparing the evolved lineages against their un-evolved ancestors. These results suggest that fungal growth and sporulation evolved through successive and exclusive use of an 
insect host as a nutritional resource. Despite our results, the use of experimental evolution to naturally adapt to a particular host could still be a good tool to study host-pathogen interactions, and understand the genetic mechanisms involved in fungal virulence. The understanding of genetic mechanisms will improve our methods to use $B$. bassiana as a viable biological control agent against malaria mosquitoes.

Chapter 6 presents a discussion on the main findings of this thesis and describes future research lines that will deepen our understanding in the key factors involved in virulence of the entomopathogenic fungus $B$. bassiana. I also address several aspects and challenges that need to be considered in order to implement EPF on a large scale as a biological control agent of malaria mosquitoes. 


\section{Acknowledgements}

The work presented in this book could not be done without the collective effort of many people that have supported me during the four years of my PhD. My PhD started as a result of collaboration among three departments: Laboratory of Genetics, Laboratory of Entomology and Laboratory of Phytopathology. At first, it was a challenge to work on three departments, but I am very glad with the journey I had while conducting this project.

I would like to thanks my promoters Willem and Bas, for guiding me through my $\mathrm{PhD}$. Willem, I am thankful for checking that everything were running smoothly and for always thinking one step further. Bas, I am grateful for your inquisitive mind, and always being there to have useful discussions of my results. Also, I appreciate your invaluable support as a career advisor. Sander, when I started my PhD I never had worked with mosquitoes before, and you were very helpful in explaining me all the techniques needed to start my experiments. I am also glad that your enthusiasm attracted many students to this project. Jan, I am truly grateful that I was able to work with you. You always have amazed me with your great knowledge in biochemistry and molecular biology of fungi. Additionally, your positive attitude was very motivating. Sijmen, your expertise with experimental evolution was a nice addition to my project and thanks for helping me measuring MGR in the lab. Fons, it was nice to discuss with you about general concepts in fungal biology, and thanks for giving me the opportunity to collaborate in MEE with a project in endophytes.

Most of my experiments were able to run smoothly due to the helpful environment in the department. Marijke, Bertha and Gabriella, thanks for your support and help in my experiments and nice atmosphere in the lab. I would like thank Sebastiaan, from Bioprocess Engineering, for helping me with mass producing spores. Also, special thanks to the rearing team in Entomology for having a constant supply of mosquitoes ready for my experiments. Wytske, thank you for taking care of all the administrative and financial work, and for being friendly and attentive.

I would like to thanks all the members of the Laboratory of Genetics for having a very relaxed atmosphere to talk about science and life. I had a lot of fun and interesting conversations while having a break or lunch, and enjoyed all the extra activities organized such as nice BBQs, laboutjes, Friday drinks and wine tastings! Likewise, I would also like to thank all the members of Entomology for nice discussions and for allowing me to join your social activities, even though I was not around that much. Also, I am grateful to the members of the Vector group for having such an interactive and dynamic weekly meeting in which we shared many ideas. As well, to the 
bioinformatics people from the Laboratory of Phytopathology for your vast patience and help in analyzing genomic data.

I am very happy that during these 4 years, I was able to meet lovely people to share nice moments in and out of the lab: Florien, Alex, Valeria, Tina, Eric, Jianhua, Vivian, Tom, Bea, Jose, Giovanni, Krystina, Esmer, Marlene, Uta, Siriluk, Chantal, Tania, André, Deborah, Cecilia, Daniel, Jelle, thank you for all the great dinners, excursions, game nights, drinks and funny occasions that we had.

I had the privilege to supervise many students during my PhD. Thank Benno, Koen, Iris, Ruben, Django, Daphne, Harm and Kamrul for helping me during my research. I have learnt a lot from you, and I enjoyed our time working together.

During my PhD I was a member of the PhD PE\&RC Council (PPC), in which I have met very nice people. It was really enjoyable to discuss about $\mathrm{PhD}$ issues, and put our bit to contribute to a better graduate school. I would like to give special thanks to Lennart and Claudius for supporting us in all our initiatives.

I would like to thank the members of the reading committee Prof. Dr. M.E. Schranz, Dr. ir V.I.D. Ros, Dr. M. Rep and Prof. Dr. J. Eilenberg, for taking time and effort to read my thesis. Valeria and Eric, thank you for being my paranymphs.

I would like to thank my family, for their unconditional support despite the distance throughout the years. Finally, I would like to thank Lidia, your support and patience are invaluable. I cannot imagine my life without you. 


\section{Curriculum Vitae}

Claudio Alberto Valero Jiménez was born on 28 October in 1985, in Maracaibo, Venezuela. In 2002, he obtained his high school degree at Colegio Bellas Artes, Maracaibo. Afterwards, he started to study Chemical Engineering, but after 1 year he realized that Biology was his passion. During his studies, he did an internship in a NGO called GTTM-GV, in which he participated in a educational project to teach marine conservation concepts in a local fishermen community. $\mathrm{He}$ also did research on the estimation of genetic diversity of fungal endophytes within trees of a tropical cloudy forest. He graduated with honours at the University of Zulia in 2010. He decided to continue his studies abroad, to pursue a Master degree in Biodiversity at the University of Barcelona, Spain. His master research was about the characterization of microsatellite markers from the invasive ascidian Styela plicata. In 2012 he started his PhD in Wageningen University at the Laboratory of Genetics and Laboratory of Entomology, which has led to this thesis. During his $\mathrm{PhD}$ research, he was an active member of the PE\&RC PhD Council (PPC), in which he helped organize the PE\&RC Day in 2013, and several social activities. After spending 4 Dutch winters, Claudio plans to return to sunny Spain. 


\section{List of publications}

Valero-Jiménez CA, Wiegers H, Zwaan BJ, Koenraadt CJM, van Kan JAL. 2016. Genes involved in virulence of the entomopathogenic fungus Beauveria bassiana. Journal of Invertebrate Pathology 133, 41-49.

Valero-Jiménez CA, Debets AJM, van Kan JAL, Schoustra SE, Takken W, Zwaan BJ, Koenraadt CJM. 2014. Natural variation in virulence of the entomopathogenic fungus Beauveria bassiana against malaria mosquitoes. Malaria journal 13 (1), 479.

Valero-Jiménez CA, Faino L, Spring in 't Veld D, Smit S, Zwaan BJ, van Kan JAL. Comparative genomics of Beauveria bassiana: uncovering signatures of virulence against insects. (Submitted to BMC Genomics).

Valero-Jiménez CA, Debets AJM, van Kan JAL, Schoustra SE, Takken W, Zwaan BJ, Koenraadt CJM. Experimental evolution to increase the efficacy of the entomopathogenic fungus Beauveria bassiana against malaria mosquitoes: effects on mycelial growth and virulence. (Under review in Journal of Evolutionary Applications).

Garcia-Cisneros A, Valero-Jiménez CA, Palacín C, Pérez-Portela R. 2013. Characterization of thirty two microsatellite loci for three Atlanto-Mediterranean echinoderm species. Conservation Genetics Resources 5 (3), 749-753.

Loro M, Valero-Jiménez CA, Nozawa S, Márquez LM. Diversity and composition of fungal endophytes in semiarid Northwest Venezuela. 2012. Journal of Arid Environments. Journal of Arid Environments 85, 46-55.

Valero-Jiménez CA, Perez-Portela R, Lopez-Legentil S. Characterization of novel microsatellite markers from the worldwide invasive ascidian Styela plicata. 2012. Conservation Genetic Resources 4 (3), 559-561. 


\section{PE\&RC Training and Education Statement}

With the training and education activities listed below the PhD candidate has complied with the requirements set by the C.T. de Wit Graduate School for Production Ecology and Resource Conservation (PE\&RC) which comprises of a minimum total of 32 ECTS (= 22 weeks of activities)

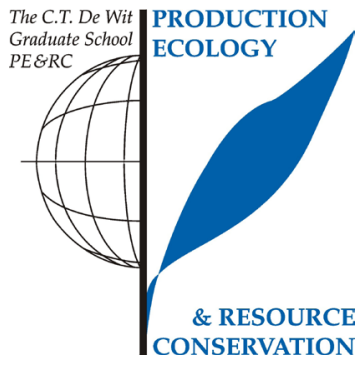

\section{Review of literature (6 ECTS)}

- Genes involved in virulence of the entomopathogenic fungus Beauveria bassiana

\section{Post-graduate courses (5.7 ECTS)}

- De novo assembly from NGS data; NBIC (2013)

- Survival analysis; PE\&RC (2013)

- Statistics using R; Imperial College London (2013)

\section{Invited review of (unpublished) journal manuscript (2 ECTS)}

- Fungal Ecology: biocontrol with entomopathogenic fungi (2014)

- Ecological Entomology: Biology of entomopathogenic fungi (2016)

\section{Deficiency, refresh, brush-up courses (6 ECTS)}

- Genomics; WUR (2012)

\section{Competence strengthening / skills courses (3.9 ECTS)}

- Competence assessment; WGS (2012)

- Scientific writing; WGS (2014)

- PhD Workshop carousel; WGS (2015)

- Biobusiness Summer School; Hyphen Projects (2015)

PE\&RC Annual meetings, seminars and the PE\&RC weekend (3.3 ECTS)

- PE\&RC First year weekend (2012)

- PE\&RC Day (2012-2016)

- PE\&RC Middle year weekend (2014)

- PE\&RC Last year weekend (2016) 


\section{Discussion groups / local seminars / other scientific meetings (8.7 ECTS)}

- 24th Annual Entomology meeting; Ede, the Netherlands (2012)

- Experimental Evolution Discussion Group (EEDG) (2012-2014)

- WEES Seminars (2012-2015)

- Annual meeting of the section Mycology of the Royal Dutch Society for Microbiology (KNVM); oral presentation; Utrecht, the Netherlands (2013)

- Evolution in the laboratory symposium; Wageningen, the Netherlands (2013)

- How to write a world-class paper symposium; Wageningen, the Netherlands (2013)

- WPC Symposium; oral presentation; Wageningen, the Netherlands (2013)

- Annual meeting of the section Mycology of the Royal Dutch Society for Microbiology (KNVM); Utrecht, the Netherlands (2015)

\section{International symposia, workshops and conferences (8.1 ECTS)}

- XIV Congress of the European Society for Evolutionary Biology; poster presentation; Lisbon, Portugal (2013)

- 12th European Conference on Fungal Genetics; poster presentation; Seville, Spain (2014)

- 28th Fungal Genetics Conference Pacific Grove; poster presentation; USA (2015)

\section{Lecturing / supervision of practicals / tutorials (12.6 ECTS)}

- Biology control of insects (2012-2015)

- Molecular Evolution Ecology (MEE) (2013, 2015)

\section{Supervision of MSc students}

- Combining entomopathogenic fungi and mosquito attractants for vector control

- Testing of novel methods to enhance virulence and production of Beauveria bassiana spores for the control of malaria vectors

- Variation in sub-lethal effects in the entomopathogenic fungus Beauveria bassiana and improving methods for infecting malaria mosquitoes with fungi

- The genetic mechanism behind the virulence variation in Beauveria bassiana

- Virulence factors in Beauveria bassiana

- Improving delivery platform of Beauveria bassiana spores by exploiting natural behavior of Anopheles coluzzii with the inclusion of heat and odour 
The research described in this thesis was financially supported by the Graduate School for Production Ecology \& Research Conservation (PE\&RC).

Photo Credits: Wim van Egmond - www.micropolitan.org

Cover design \& Layout: Claudio A. Valero Jiménez

Printed by: Proefschriftmaken.nl - Digiforce Vianen 
